Supporting Information For

\title{
Enantioselective Total Syntheses of Pentacyclic Homoproaporphine Alkaloids
}

Liu-Yang Pu, Fan Yang, Ji-Qiang Chen, Ying Xiong, Huai-Yu Bin, Jian-Hua Xie*, Qi-Lin Zhou, State Key Laboratory and Institute of Elemento-organic Chemistry, College of Chemistry, Nankai University, Tianjin 300071, China

Email: jhxie@nankai.edu.cn; qlzhou@nankai.edu.cn

Table of Contents
A) General Information.
B) Synthesis of Enone Intermediate $(+)-11$.
C) Synthesis of Ketone Intermediate (+)-10.
D) Synthesis of Intermediate (-)-9 and Pentacyclic Homoproaporphine Alkaloids. . .513
E) NMR Spectra of New Compounds and Pentacyclic Homoproaporphine Alkaloids. .522
F) HPLC Charts for $(-)-13 \ldots$ . $\mathbf{S 4 5}$ 


\section{A) General Information}

All reactions and manipulations which are sensitive to moisture or air were performed under inert atmosphere of argon. All chemicals were purchased from J\&K, TCI and Aldrich, and were used as received. Tianjin. Petroleum ether (PE) refers to the fraction boiling in the $60-90{ }^{\circ} \mathrm{C}$ range. Anhydrous THF, $\mathrm{Et}_{2} \mathrm{O}$ was distilled from sodium benzophenone ketyl. Anhydrous $\mathrm{CH}_{2} \mathrm{Cl}_{2}, \mathrm{Et}_{3} \mathrm{~N}$, DMSO and $i \mathrm{PrOH}$ were distilled from calcium hydride. NMR spectra were recorded on a Bruker AV 400 spectrometer at $400 \mathrm{MHz}\left({ }^{1} \mathrm{H}\right.$ NMR $), 101 \mathrm{MHz}\left({ }^{13} \mathrm{C}\right.$ NMR). Chemical shifts were reported in ppm relative to internal TMS for ${ }^{1} \mathrm{H}$ NMR data, deuterated solvent for ${ }^{13} \mathrm{C}$ NMR data, respectively. Data are presented in the following space: chemical shift, multiplicity, coupling constant in hertz (Hz), and signal area integration in natural numbers. Optical rotations were determined using a Perkin Elmer 341 polarimeter. HRMS were recorded on APEXII and ZAB-HS spectrometer. High-resolution mass spectra were recorded on an IonSpec FT-ICR mass spectrometer. HPLC analyses were determined using a Hewlett Packard Model HP 1100 Series chromatography. 


\section{B) Synthesis of Enone Intermediate (+)-11}

Enone intermediate $(+)-\mathbf{1 1}$ was synthesized from tricyclic benzofuran $(-)-8$ by the following procedure.
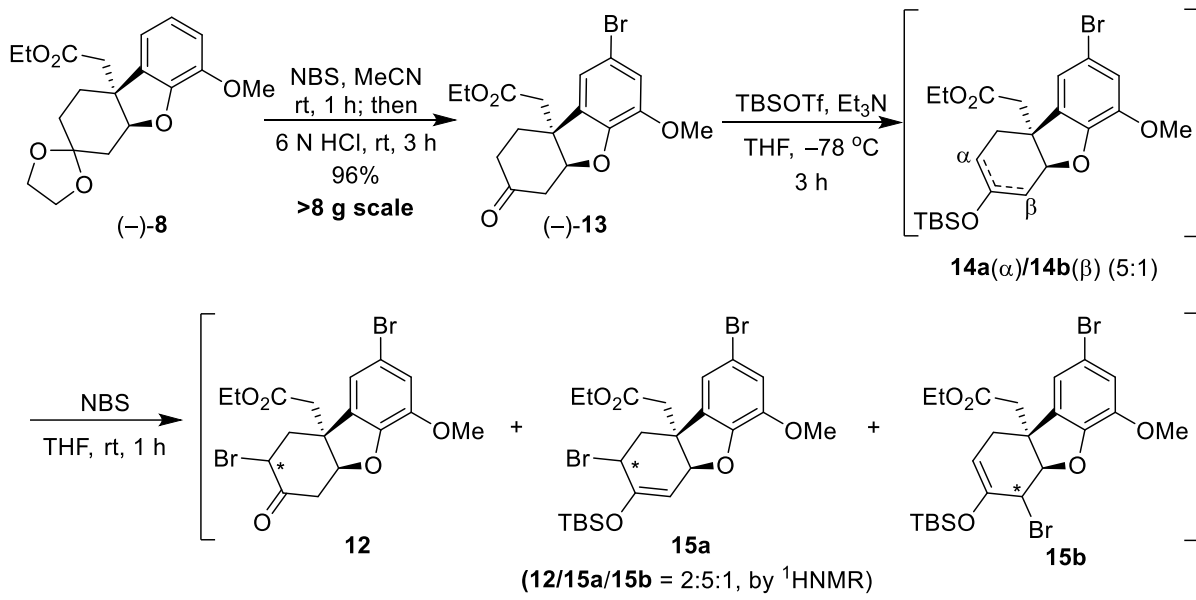

$14 a(\alpha) / 14 b(\beta)(5: 1)$

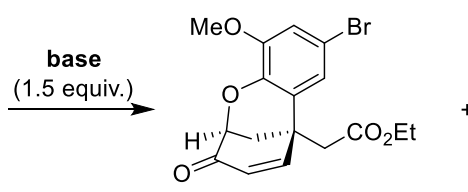

$(+)-11$<smiles>COCCC12C=CC(=O)CC1Oc1c(OC)cc(Br)cc12</smiles>

16 (by product)

Tricyclic benzofuran (-)-8 was synthesized according to our previously developed method in five steps in around $70 \%$ overall yields and $93 \%$ ee on a multi-gram scale from commercially available 7-bromo-1,4-dioxaspiro[4.5]decan-8-one and 2-iodo-6-methoxyphenol. ${ }^{1}$

\section{Synthesis of compound (-)-13}
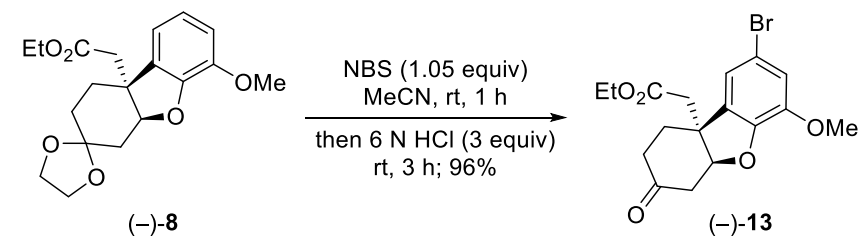

To a solution of compound (-)-8 $(8.60 \mathrm{~g}, 24.7 \mathrm{mmol})$ in $100 \mathrm{~mL} \mathrm{MeCN}$ was added NBS (4.76 g, $25.9 \mathrm{mmol}$ ) slowly in small portions at room temperature, and the resulting mixture was stirred for $1 \mathrm{~h}$ to complete the reaction. Then saturated aqueous solution of $\mathrm{Na}_{2} \mathrm{~S}_{2} \mathrm{O}_{3}(20 \mathrm{~mL})$ was added to quench the reaction. Subsequently, aqueous $6 \mathrm{~N} \mathrm{HCl}$ solution $(12 \mathrm{~mL})$ was added to the mixture and stirred at room temperature for another $3 \mathrm{~h}$. After neutralization with saturated aqueous $\mathrm{NaHCO}_{3}$ solution $(150 \mathrm{~mL})$, the mixture was extracted with EtOAc $(3 \times 60 \mathrm{~mL})$. The combined organic phase was dried over anhydrous $\mathrm{Na}_{2} \mathrm{SO}_{4}$ and concentrated in vacuo. The residue was chromatographed on silica-gel column with petroleum ether/ethyl acetate (3:1) to give the product $(-)-\mathbf{1 3}(8.90 \mathrm{~g}, 96 \%$ yield) as a white solid. $6.70 \mathrm{~g}$ white crystal was yielded after recrystallization from $\mathrm{Et}_{2} \mathrm{O}, 72 \%$ recrystallization yield, mp 99$101{ }^{\circ} \mathrm{C}, 99.5 \%$ ee; HPLC conditions: column, Chiralcel OJ-3; eluent, 2-propanol/hexane 20:80; temp, $25^{\circ} \mathrm{C}$; flow rate, $1.0 \mathrm{~mL} / \mathrm{min}$; detection, $210 \mathrm{~nm}$ light; $R_{\mathrm{f}} 0.5$ (petroleum ether/ethyl acetate $\left.=2: 1\right) ;[\alpha]_{\mathrm{D}}^{25}$ -162.6 (c 1.0, $\left.\mathrm{CHCl}_{3}\right)$; IR (KBr): $v_{\max }=2936,1723,1616,1486,1444,1202,1026,838,735 \mathrm{~cm}^{-1} ;{ }^{1} \mathrm{H}$ NMR $\left(400 \mathrm{MHz}, \mathrm{CDCl}_{3}\right) \delta: 6.88(\mathrm{~d}, J=1.6 \mathrm{~Hz}, 1 \mathrm{H}), 6.86(\mathrm{~d}, J=1.6 \mathrm{~Hz}, 1 \mathrm{H}), 5.26(\mathrm{t}, J=3.2 \mathrm{~Hz}, 1 \mathrm{H})$, 4.14 (q, $J=7.2 \mathrm{~Hz}, 2 \mathrm{H}), 3.84(\mathrm{~s}, 3 \mathrm{H}), 3.03(\mathrm{dd}, J=17.2,3.2 \mathrm{~Hz}, 1 \mathrm{H}), 2.97(\mathrm{dd}, J=17.2,3.2 \mathrm{~Hz}, 1 \mathrm{H})$, 
$2.86(\mathrm{~d}, J=16.0 \mathrm{~Hz}, 1 \mathrm{H}), 2.81(\mathrm{~d}, J=16.0 \mathrm{~Hz}, 1 \mathrm{H}), 2.34-2.25(\mathrm{~m}, 2 \mathrm{H}), 1.98-1.87(\mathrm{~m}, 2 \mathrm{H}), 1.24(\mathrm{t}, J=$ $7.2 \mathrm{~Hz}, 3 \mathrm{H}) ;{ }^{13} \mathrm{C}$ NMR $\left(101 \mathrm{MHz}, \mathrm{CDCl}_{3}\right) \delta: 208.4,170.4,146.6,144.9,133.8,118.3,115.5,112.9$, 86.9, 61.0, 56.2, 46.6, 44.2, 42.2, 35.6, 31.2, 14.2; HRMS $(m / z)$ : calcd for $\mathrm{C}_{17} \mathrm{H}_{20} \mathrm{BrO}_{5}\left([\mathrm{M}+\mathrm{H}]^{+}\right)$ 383.0489 , found 383.0478 .

\section{Synthesis of enone intermediate $(+)-11$ in a one-pot procedure}

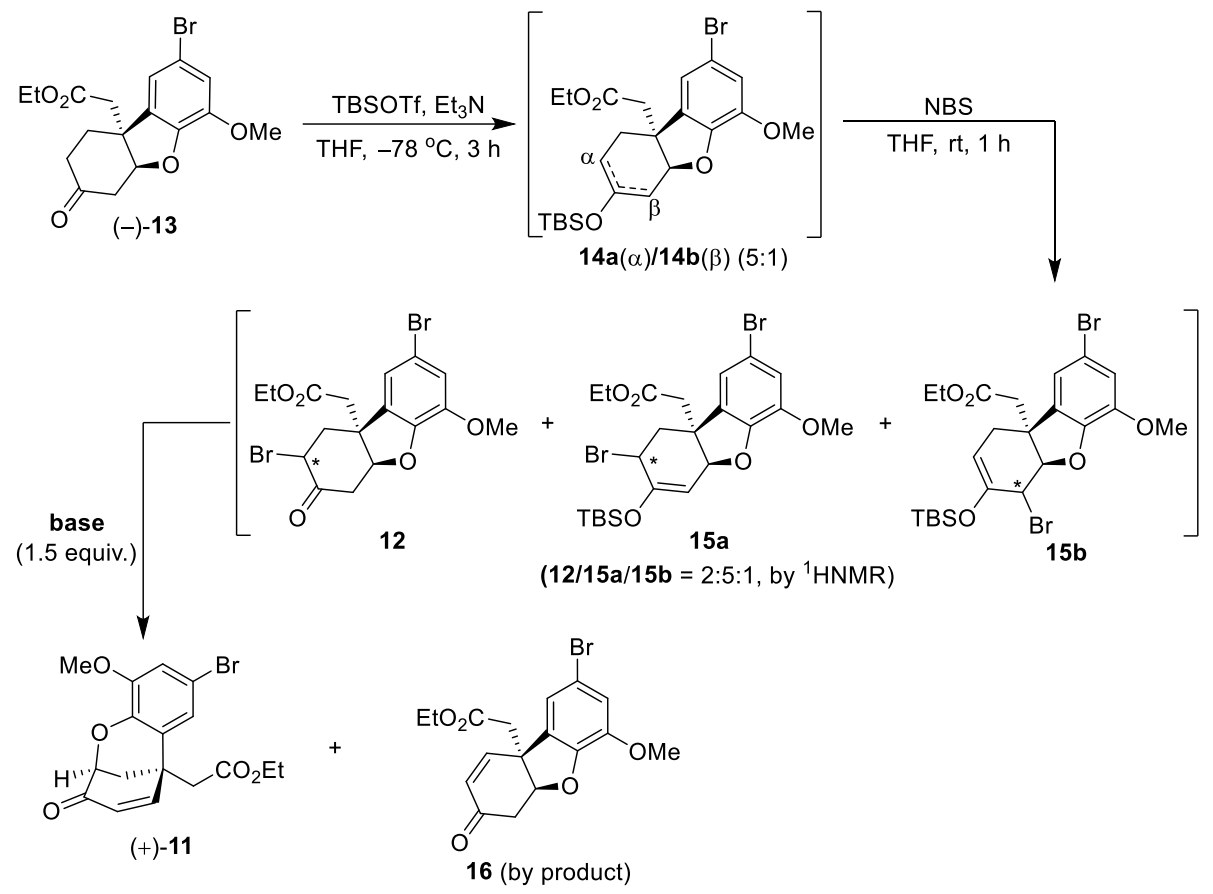

\section{Screening of the bases}

To a solution of compound (-)-13 $(0.19 \mathrm{~g}, 0.50 \mathrm{mmol})$ in THF $(1.5 \mathrm{~mL})$ at $-78{ }^{\circ} \mathrm{C}$ was added $\mathrm{Et}_{3} \mathrm{~N}$ $(0.23 \mathrm{~mL}, 1.70 \mathrm{mmol})$, then TBSOTf $(0.25 \mathrm{~mL}, 1.10 \mathrm{mmol})$ was added dropwise within $5 \mathrm{~min}$. The mixture was stirred at $-78{ }^{\circ} \mathrm{C}$ for $3 \mathrm{~h}$ to complete the reaction and then concentrated in high vacuo. The resulting colorless oil was dissolved in THF $(1.5 \mathrm{~mL})$ and treated with a solution of NBS $(0.11 \mathrm{~g}, 0.60$ $\mathrm{mmol})$ in THF $(1.5 \mathrm{~mL})$ dropwise. The mixture was stirred at room temperature for another $1 \mathrm{~h}$. Then to the mixture was added base $(0.80 \mathrm{mmol})$ in THF $(0.80 \mathrm{~mL})$ dropwise. The resulting mixture was stirred at room temperature for $2-20 \mathrm{~h}$ and quenched with saturated aqueous $\mathrm{NaHCO}_{3}$ solution $(5 \mathrm{~mL})$ and extracted with $\mathrm{Et}_{2} \mathrm{O}(3 \times 5 \mathrm{~mL})$. The combined organic phase was dried over $\mathrm{Na}_{2} \mathrm{SO}_{4}$ and concentrated in vacuo. The residue was purified by column chromatography on silica gel with petroleum ether/ethyl acetate (3:1) to give a mixture of product $(+)-\mathbf{1 1}\left(R_{\mathrm{f}} 0.37\right)$ and by-product $16\left(R_{\mathrm{f}}\right.$ 0.37 ) as a white solid. The results are summarized in Table S1. 
Table S1. The results of enantioselective synthesis of (+)-11 in a one-pot procedure. ${ }^{a}$

\begin{tabular}{cccccc}
\hline Entry & Base & Temp $\left({ }^{\circ} \mathrm{C}\right)$ & Time $(\mathrm{h})$ & $(+)-\mathbf{1 1 / 1 \mathbf { 1 6 } ^ { b }}$ & ${\text { Yield }(\%)^{\mathrm{c}}}^{\mathrm{c}}$ \\
\hline 1 & $\mathrm{DBU}$ & $\mathrm{rt}$ & 15 & $94: 6$ & 58 \\
2 & $t$ BuOK & $\mathrm{rt}$ & 20 & $97: 3$ & 37 \\
3 & $\mathrm{DABCO}$ & 50 & 10 & $96: 4$ & 41 \\
4 & $\mathrm{Et}_{3} \mathrm{~N}$ & 50 & 10 & $99: 1$ & 27 \\
5 & $\mathrm{TBAF}$ & $\mathrm{rt}$ & 2 & $>99: 1$ & 69 \\
$6^{[\mathrm{d}]}$ & $\mathrm{TBAF}$ & $\mathrm{rt}$ & 2 & $>99: 1$ & 70 \\
\hline
\end{tabular}

${ }^{a}$ Reaction conditions: (-)-13 (0.19 g, $\left.0.50 \mathrm{mmol}\right), 1.5$ equivalent of base.

${ }^{b}$ The ratio of (+)-11 to $\mathbf{1 6}$ was ditermind by ${ }^{1} \mathrm{H}$ NMR.

${ }^{c}$ The yield of (+)-11 was caculated from the combined isolated yield of (+)-11 and $\mathbf{1 6}$ over 3 steps.

${ }^{d}$ Performed on a gram-scale $((-)-\mathbf{1 3}, 1.15 \mathrm{~g}, 3.00 \mathrm{mmol})$.

\section{Gram-scale synthesis with TBAF as a base}

To a solution of compound $(-)-\mathbf{1 3}(1.15 \mathrm{~g}, 3.00 \mathrm{mmol})$ in THF $(9.0 \mathrm{~mL})$ at $-78{ }^{\circ} \mathrm{C}$ was added $\mathrm{Et}_{3} \mathrm{~N}$ $(1.37 \mathrm{~mL}, 9.90 \mathrm{mmol})$, TBSOTf $(1.52 \mathrm{~mL}, 6.60 \mathrm{mmol})$ was added dropwise within $15 \mathrm{~min}$. The mixture was stirred at $-78^{\circ} \mathrm{C}$ for $3 \mathrm{~h}$ to complete the reaction and then concentrated in high vacuo. The resulting colorless oil was dissolved in THF $(9.0 \mathrm{~mL})$ and treated with a solution of NBS $(0.58 \mathrm{~g}, 3.15$ mmol) in THF $(9.0 \mathrm{~mL})$ dropwise. The mixture was stirred at room temperature for another $1 \mathrm{~h}$. Then to the mixture was added TBAF $(4.70 \mathrm{~mL}, 4.70 \mathrm{mmol}, 1.0 \mathrm{M}$ in THF) dropwise. The resulting mixture was stirred at room temperature for $2 \mathrm{~h}$ and quenched with saturated aqueous $\mathrm{NaHCO}_{3}$ solution $(15 \mathrm{~mL})$ and extracted with $\mathrm{Et}_{2} \mathrm{O}(3 \times 15 \mathrm{~mL})$. The combined organic phase was dried over $\mathrm{Na}_{2} \mathrm{SO}_{4}$ and concentrated in vacuo. The residue was purified by column chromatography on silica gel with petroleum ether/ethyl acetate (3:1) to give the product $(+)-\mathbf{1 1}(0.82 \mathrm{~g}, 70 \%$ yield $)$ as a white solid. $\mathrm{mp}$ $77-79^{\circ} \mathrm{C} ; R_{\mathrm{f}} 0.37$ (petroleum ether/ethyl acetate $=3: 1$ ).

\section{Treatment of the isolated intermediates $12,15 a$, and $15 b$ with TBAF}<smiles>CCOC(=O)CC12CC(Br)C(=O)CC1Oc1c(OC)cc(Br)cc12</smiles>

12

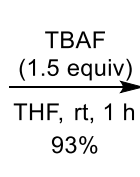

\% $\%$<smiles>CCOC(=O)CC12C=CC(=O)C(CC1=O)Oc1c(OC)cc(Br)cc12</smiles>

$(+)-11$

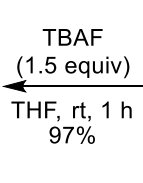

$97 \%$

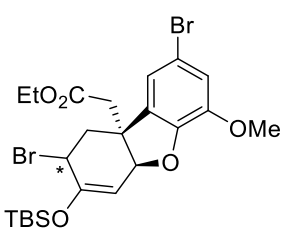

$15 a$

General procedure: To a solution of the isolated bromide intermediate $(27.0 \mu \mathrm{mol})$ THF $(0.20$ $\mathrm{mL}$ ) was added TBAF ( $40.0 \mu \mathrm{L}, 40.0 \mu \mathrm{mol}, 1.0 \mathrm{M}$ in THF) was added dropwise. The resulting mixture was stirred at room temperature for $1 \mathrm{~h}$ and quenched with saturated aqueous $\mathrm{NaHCO}_{3}$ solution $(3 \mathrm{~mL})$ and extracted with $\mathrm{Et}_{2} \mathrm{O}(3 \times 3 \mathrm{~mL})$. The combined organic phase was dried over $\mathrm{Na}_{2} \mathrm{SO}_{4}$ and concentrated in vacuo. The residue was purified by column chromatography on silica gel with petroleum ether/ethyl acetate (3:1) to give the product as a white solid.

For intermediate 12, the product $(+)-\mathbf{1 1}(9.6 \mathrm{mg})$ was obtained in $93 \%$ yield.

For intermediate 15a, the product $(+)-\mathbf{1 1}(10.0 \mathrm{mg})$ was obtained in $97 \%$ yield.

For intermediate 15b, only complex mixtures without any detectable $(+)-11$ were obtained. 
The data of the isolated intermediates $(\mathbf{1 4}, \mathbf{1 2}, \mathbf{1 5 a}$, and 15b) and product $(+)-\mathbf{1 1}$ as below:

14a/14b (5:1): colorless oil $\left(R_{\mathrm{f}} 0.63\right.$, petroleum ether/ethyl acetate $\left.=20: 1\right) .{ }^{1} \mathrm{H} \mathrm{NMR}(400 \mathrm{MHz}$, $\left.\mathrm{CDCl}_{3}\right) \delta: 6.91(\mathrm{~d}, J=1.6 \mathrm{~Hz}, 0.17 \mathrm{H}), 6.86(\mathrm{~d}, J=1.6 \mathrm{~Hz}, 0.17 \mathrm{H}), 6.82(\mathrm{~d}, J=2.0 \mathrm{~Hz}, 0.83 \mathrm{H}), 6.81(\mathrm{~d}$, $J=2.0 \mathrm{~Hz}, 0.83 \mathrm{H}), 5.24(\mathrm{~d}, J=4.0 \mathrm{~Hz}, 0.17 \mathrm{H}), 5.18(\mathrm{dd}, J=4.8,3.2 \mathrm{~Hz}, 0.83 \mathrm{H}), 4.98(\mathrm{~d}, J=4.0 \mathrm{~Hz}$, $0.17 \mathrm{H}), 4.74-4.70(\mathrm{~m}, 0.83 \mathrm{H}), 4.10(\mathrm{q}, J=7.2 \mathrm{~Hz}, 0.34 \mathrm{H}), 4.09(\mathrm{q}, J=7.2 \mathrm{~Hz}, 1.66 \mathrm{H}), 3.84(\mathrm{~s}, 0.51 \mathrm{H})$, $3.82(\mathrm{~s}, 2.49 \mathrm{H}), 2.69(\mathrm{~s}, 1.66 \mathrm{H}), 2.63(\mathrm{dd}, J=16.0,4.8 \mathrm{~Hz}, 1 \mathrm{H}), 2.57(\mathrm{~d}, J=5.6 \mathrm{~Hz}, 0.34 \mathrm{H}), 2.51(\mathrm{dd}, J$ $=16.0,3.2 \mathrm{~Hz}, 1 \mathrm{H}), 2.36(\mathrm{dd}, J=15.2,3.2 \mathrm{~Hz}, 1 \mathrm{H}), 2.27(\mathrm{dd}, J=15.2,2.4 \mathrm{~Hz}, 1 \mathrm{H}), 2.15-1.93(\mathrm{~m}, 1 \mathrm{H})$, $1.21(\mathrm{t}, J=7.2 \mathrm{~Hz}, 0.51 \mathrm{H}), 1.20(\mathrm{t}, J=7.2 \mathrm{~Hz}, 2.49 \mathrm{H}), 0.89-0.84(\mathrm{~m}, 9 \mathrm{H}), 0.11-0.00(\mathrm{~m}, 6 \mathrm{H}) ;{ }^{13} \mathrm{C}$ NMR (101 MHz, $\left.\mathrm{CDCl}_{3}\right) \delta: 170.7,170.5,156.8,150.3,147.4,146.3,145.8,144.6,136.1,135.0,118.7$, 118.3, 118.0, 115.0, 114.6, 111.8, 101.3, 100.2, 89.0, 86.8, 60.6, 56.2, 56.1, 48.3, 45.6, 44.2, 42.1, 34.9, $33.4,29.2,26.8,25.6,18.0,14.1,-4.5(2),-4.6,-4.9 ;$ HRMS $(m / z)$ : calcd for $\mathrm{C}_{23} \mathrm{H}_{34} \mathrm{BrO}_{5} \mathrm{Si}\left([\mathrm{M}+\mathrm{H}]^{+}\right)$ 498.5085, found 498.5082 .

12 (unstable, with minor isomers or other impurities): yellow oil ( $R_{\mathrm{f}} 0.4$, petroleum ether/ethyl acetate = 3:1). ${ }^{1} \mathrm{H}$ NMR $\left(400 \mathrm{MHz} \mathrm{CDCl}_{3}\right) \delta: 6.93(\mathrm{~d}, J=1.6 \mathrm{~Hz}, 1 \mathrm{H}), 6.91(\mathrm{~d}, J=1.6 \mathrm{~Hz}, 1 \mathrm{H}), 5.24(\mathrm{t}$, $J=3.2 \mathrm{~Hz}, 1 \mathrm{H}), 4.16$ (q, $J=7.2 \mathrm{~Hz}, 2 \mathrm{H}), 4.11(\mathrm{dd}, J=14.0,4.8 \mathrm{~Hz}, 1 \mathrm{H}), 3.85$ (s, 3H), 3.27 (dd, $J=$ 15.6, 3.2 Hz, 1H), $3.17(\mathrm{dd}, J=15.6,3.2 \mathrm{~Hz}, 1 \mathrm{H}), 2.90$ (d, $J=16.4 \mathrm{~Hz}, 1 \mathrm{H}), 2.81(\mathrm{~d}, J=16.4 \mathrm{~Hz}, 1 \mathrm{H})$, $2.77(\mathrm{t}, J=14.0 \mathrm{~Hz}, 1 \mathrm{H}), 2.48(\mathrm{dd}, J=15.6,4.8 \mathrm{~Hz}, 1 \mathrm{H}), 1.26(\mathrm{t}, J=7.2 \mathrm{~Hz}, 3 \mathrm{H}) ;{ }^{13} \mathrm{C} \mathrm{NMR}(101 \mathrm{MHz}$, $\left.\mathrm{CDCl}_{3}\right) \delta: 200.6,170.2,146.2,145.2,132.8,118.3,116.0,113.7,86.4,61.3,56.4,48.5,46.9,43.6,42.6$, 41.5, 14.2; HRMS $(m / z)$ : calcd for $\mathrm{C}_{17} \mathrm{H}_{19} \mathrm{Br}_{2} \mathrm{O}_{5}\left([\mathrm{M}+\mathrm{H}]^{+}\right) 463.1415$, found 463.1412.

15a: yellow oil $\left(R_{\mathrm{f}} 0.6\right.$, petroleum ether/ethyl acetate $\left.=20: 1\right) .{ }^{1} \mathrm{H}$ NMR $\left(400 \mathrm{MHz}, \mathrm{CDCl}_{3}\right) \delta: 7.02$ $(\mathrm{d}, J=2.0 \mathrm{~Hz}, 1 \mathrm{H}), 6.88(\mathrm{~d}, J=2.0 \mathrm{~Hz}, 1 \mathrm{H}), 5.31(\mathrm{dd}, J=4.4,0.8 \mathrm{~Hz}, 1 \mathrm{H}), 5.11(\mathrm{dd}, J=4.4,0.8 \mathrm{~Hz}$, $1 \mathrm{H}), 4.47(\mathrm{t}, J=6.0 \mathrm{~Hz}, 1 \mathrm{H}), 4.17-4.07(\mathrm{~m}, 2 \mathrm{H}), 3.84(\mathrm{~s}, 3 \mathrm{H}), 2.82(\mathrm{~d}, J=16.0 \mathrm{~Hz}, 1 \mathrm{H}), 2.74(\mathrm{~d}, J=$ $16.0 \mathrm{~Hz}, 1 \mathrm{H}), 2.69$ (d, $J=6.0 \mathrm{~Hz}, 2 \mathrm{H}), 1.21(\mathrm{t}, J=7.2 \mathrm{~Hz}, 3 \mathrm{H}), 0.91(\mathrm{~s}, 9 \mathrm{H}), 0.18(\mathrm{~s}, 3 \mathrm{H}), 0.10(\mathrm{~s}, 3 \mathrm{H})$; ${ }^{13} \mathrm{C}$ NMR $\left(101 \mathrm{MHz}, \mathrm{CDCl}_{3}\right) \delta: 170.2,153.3,145.8,145.7,134.5,118.8,115.3,112.6,102.9,85.6$, $60.7,56.2,45.9,44.7,41.5,38.8,25.5,18.2,14.2,-4.7,-4.8$; HRMS $(m / z)$ : calcd for $\mathrm{C}_{23} \mathrm{H}_{33} \mathrm{Br}_{2} \mathrm{O}_{5} \mathrm{Si}$ $\left([\mathrm{M}+\mathrm{H}]^{+}\right) 577.4045$, found 577.4038 .

15b: yellow oil $\left(R_{\mathrm{f}} 0.65\right.$, petroleum ether/ethyl acetate $\left.=20: 1\right) .{ }^{1} \mathrm{H}$ NMR $\left(400 \mathrm{MHz}, \mathrm{CDCl}_{3}\right) \delta$ : $6.86(\mathrm{~d}, J=1.6 \mathrm{~Hz}, 1 \mathrm{H}), 6.82(\mathrm{~d}, J=1.6 \mathrm{~Hz}, 1 \mathrm{H}), 5.49$ (d, $J=2.0 \mathrm{~Hz}, 1 \mathrm{H}), 4.93(\mathrm{~d}, J=7.6 \mathrm{~Hz}, 1 \mathrm{H})$, $4.58-4.53(\mathrm{~m}, 1 \mathrm{H}), 4.09-4.01(\mathrm{~m}, 2 \mathrm{H}), 3.82(\mathrm{~s}, 3 \mathrm{H}), 2.85(\mathrm{~s}, 2 \mathrm{H}), 2.64(\mathrm{dd}, J=15.6,2.8 \mathrm{~Hz}, 1 \mathrm{H}), 2.54$ $(\mathrm{dd}, J=15.6,7.6 \mathrm{~Hz}, 1 \mathrm{H}), 1.13(\mathrm{t}, J=7.2 \mathrm{~Hz}, 3 \mathrm{H}), 0.88(\mathrm{~s}, 9 \mathrm{H}), 0.06(\mathrm{~s}, 3 \mathrm{H}), 0.03(\mathrm{~s}, 3 \mathrm{H}) ;{ }^{13} \mathrm{C} \mathrm{NMR}$ $\left(101 \mathrm{MHz}, \mathrm{CDCl}_{3}\right) \delta: 169.8,150.5,146.8,144.2,135.1,118.1,115.0,112.4,106.0,91.5,60.7,56.1$, 48.2, 46.8, 46.0, 33.9, 25.6, 18.0, 14.0, -4.7, -4.8; HRMS $(m / z)$ : calcd for $\mathrm{C}_{23} \mathrm{H}_{33} \mathrm{Br}_{2} \mathrm{O}_{5} \mathrm{Si}\left([\mathrm{M}+\mathrm{H}]^{+}\right)$ 577.4045, found 577.4040 .

$(+)-11$ : a white solid $\left(R_{\mathrm{f}} 0.37\right.$, petroleum ether/ethyl acetate $\left.=3: 1\right) . \mathrm{mp} 76-79{ }^{\circ} \mathrm{C} ;[\alpha]_{\mathrm{D}}^{25}+250.0(c$ $0.1, \mathrm{CHCl}_{3}$ ); IR (KBr): $v_{\max }=2978,2939,1729,1688,1573,1472,1445,1268,1207,1037,860,822$ $\mathrm{cm}^{-1} ;{ }^{1} \mathrm{H}$ NMR $\left(400 \mathrm{MHz}, \mathrm{CDCl}_{3}\right) \delta: 7.01(\mathrm{dd}, J=10.0,2.0 \mathrm{~Hz}, 1 \mathrm{H}), 6.95(\mathrm{~s}, 2 \mathrm{H}), 6.14(\mathrm{dd}, J=10.0$, $0.8 \mathrm{~Hz}, 1 \mathrm{H}), 4.88-4.85(\mathrm{~m}, 1 \mathrm{H}), 4.17$ (q, $J=7.2 \mathrm{~Hz}, 2 \mathrm{H}), 3.84(\mathrm{~s}, 3 \mathrm{H}), 3.17$ (d, $J=15.2 \mathrm{~Hz}, 1 \mathrm{H}), 2.81$ $(\mathrm{d}, J=15.2 \mathrm{~Hz}, 1 \mathrm{H}), 2.59(\mathrm{dd}, J=13.6,1.6 \mathrm{~Hz}, 1 \mathrm{H}), 2.35(\mathrm{ddd}, J=13.6,4.0,2.4 \mathrm{~Hz}, 1 \mathrm{H}), 1.27(\mathrm{t}, J=$ $7.2 \mathrm{~Hz}, 3 \mathrm{H}) ;{ }^{13} \mathrm{C} \mathrm{NMR}\left(101 \mathrm{MHz}, \mathrm{CDCl}_{3}\right) \delta: 191.2,169.8,156.9,149.7,141.1,127.5,126.3,118.5$, 114.6, 112.5, 74.9, 61.1, 56.3, 41.2, 35.1, 32.6, 14.2; HRMS $(m / z)$ : calcd for $\mathrm{C}_{17} \mathrm{H}_{18} \mathrm{BrO}_{5}\left([\mathrm{M}+\mathrm{H}]^{+}\right)$ 381.0332 , found 381.0334 . 
The ratio of $\mathbf{1 4 a} / \mathbf{1 4 b}$ was determined by ${ }^{1} \mathrm{H}$ NMR and varied over time.

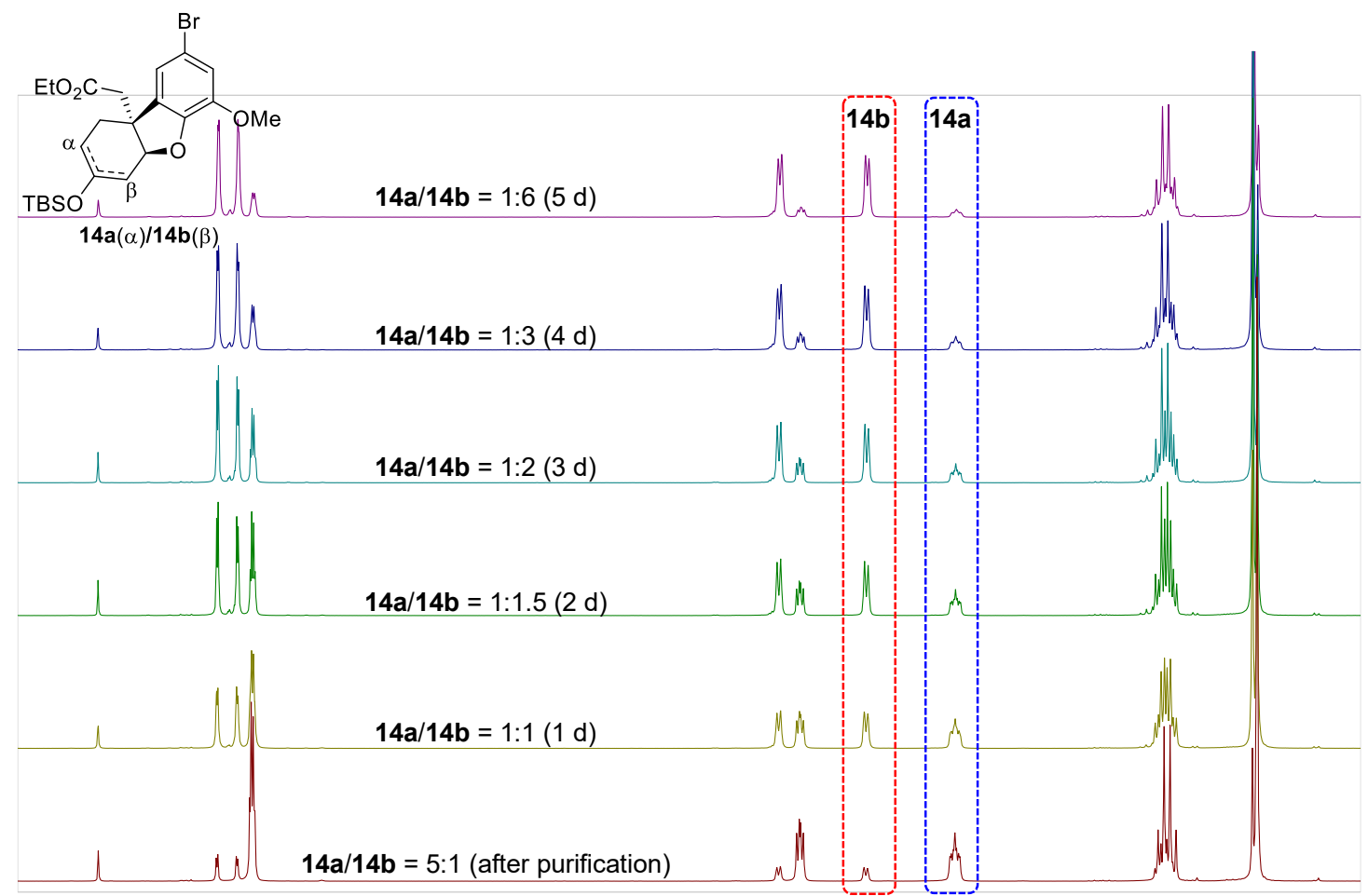

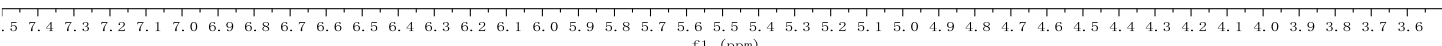

The ratio of $\mathbf{1 2} / \mathbf{1 5} \mathbf{a} / \mathbf{1 5} \mathbf{b}$ was determined by ${ }^{1} \mathrm{H}$ NMR.
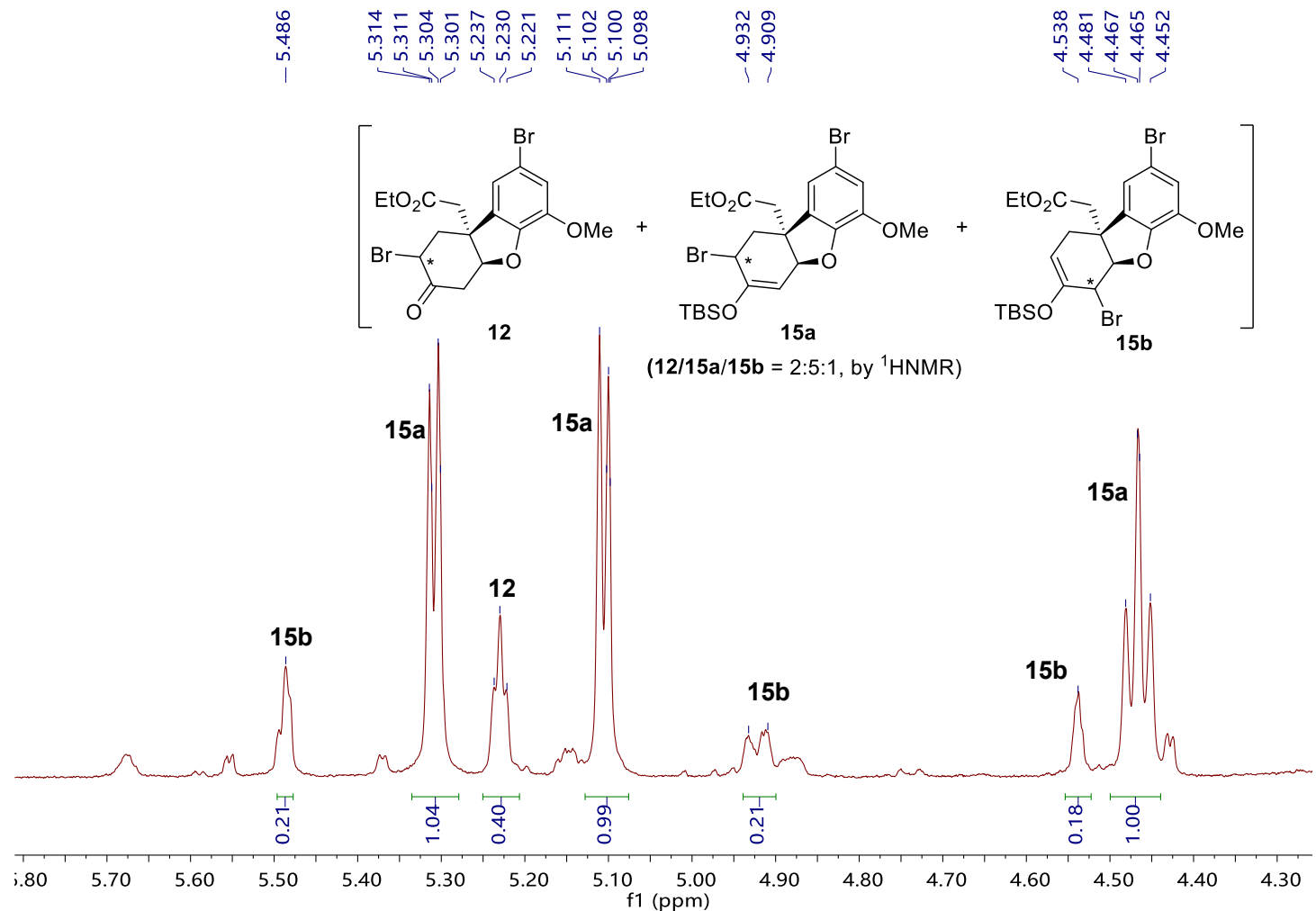
Synthesis of enone intermediate $(+)-11$ from purified intermediates<smiles>CCOC(=O)CC12CCC(=O)CC1Oc1c(OC)cc(Br)cc12</smiles>

$(-)-13$

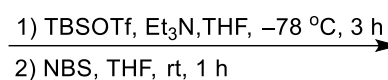

3) TBAF, THF, rt, $2 \mathrm{~h}$

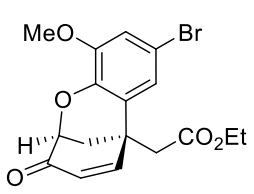

(+)-11

To a solution of compound (-)-13 $(5.90 \mathrm{~g}, 15.4 \mathrm{mmol})$ in THF $(77 \mathrm{~mL})$ at $-78{ }^{\circ} \mathrm{C}$ was added $\mathrm{Et}_{3} \mathrm{~N}$ $(7.03 \mathrm{~mL}, 50.8 \mathrm{mmol})$, and TBSOTf $(7.78 \mathrm{~mL}, 33.9 \mathrm{mmol})$ was added dropwise within $10 \mathrm{~min}$. The mixture was stirred at $-78{ }^{\circ} \mathrm{C}$ for $3 \mathrm{~h}$ to complete the reaction. Water $(50 \mathrm{~mL})$ was added to quench the reaction and the mixture was extracted with $\mathrm{Et}_{2} \mathrm{O}(3 \times 40 \mathrm{~mL})$. The combined organic phase was dried over $\mathrm{Na}_{2} \mathrm{SO}_{4}$ and concentrated in vacuo. The residue was flash chromatographed on silica-gel with petroleum ether/ethyl acetate (3:1) to give a colorless oil. The colorless oil was re-dissolved in THF (60 $\mathrm{mL})$ and cooled to $0{ }^{\circ} \mathrm{C}$ with an ice-bath. A solution of NBS $(2.97 \mathrm{~g}, 16.2 \mathrm{mmol})$ in THF $(80 \mathrm{~mL})$ was then added dropwise at $0{ }^{\circ} \mathrm{C}$, and the resulting reaction mixture was stirred at room temperature for another $1 \mathrm{~h}$. The reaction mixture was then quenched with saturated aqueous $\mathrm{Na}_{2} \mathrm{~S}_{2} \mathrm{O}_{3}$ solution $(50 \mathrm{~mL})$ and extracted with $\mathrm{Et}_{2} \mathrm{O}(3 \times 50 \mathrm{~mL})$. The combined organic phase was dried over $\mathrm{Na}_{2} \mathrm{SO}_{4}$ and concentrated in vacuo. The residue was flash chromatographed on a silica-gel column with petroleum ether/ethyl acetate (3:1) to give a yellow oil. The yellow oil was re-dissolved in THF (60 $\mathrm{mL})$, and TBAF (23.1 mL, $23.1 \mathrm{mmol}, 1.0 \mathrm{M}$ in THF) was added dropwise. The resulting mixture was stirred at room temperature for $2 \mathrm{~h}$ and quenched with saturated aqueous $\mathrm{NaHCO}_{3}$ solution $(30 \mathrm{~mL})$ and extracted with $\mathrm{Et}_{2} \mathrm{O}(3 \times 60 \mathrm{~mL})$. The combined organic phase was dried over $\mathrm{Na}_{2} \mathrm{SO}_{4}$ and concentrated in vacuo. The residue was purified by column chromatography on silica gel (petroleum ether/ethyl acetate, $3: 1)$ to give the product $(+)-11(5.05 \mathrm{~g}, 86 \%$ yield $)$ as a white solid. 


\section{C) Enantioselective Synthesis of Ketone Intermediate $(+)-10$}

The ketone intermediate $(+)-\mathbf{1 0}$ was synthesized by the following procedure.

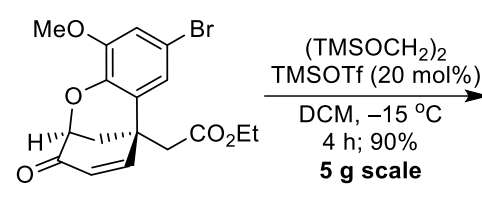

(+)-11

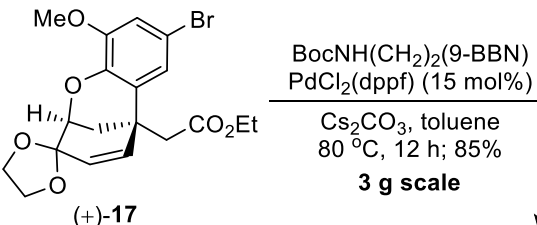

$(+)-17$

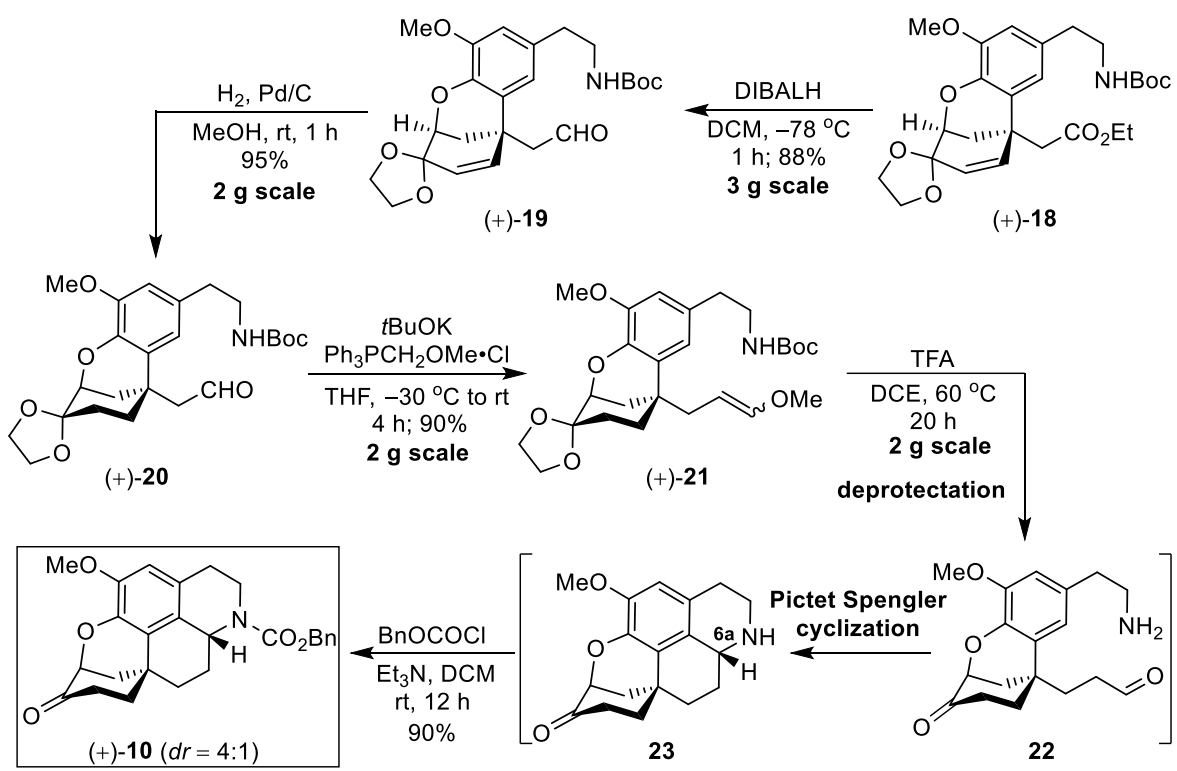

\section{Synthesis of compound $(+)-17$}<smiles>CCOC(=O)C1CC2CCCC1c1cc(Br)cc(Br)c1OC2=O</smiles>

(+)-11

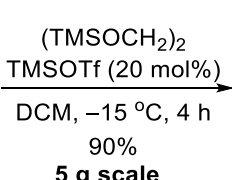

5 g scale

To a solution of compound (+)-11 (5.20 g, $13.6 \mathrm{mmol})$ and 2,2,7,7-tetramethyl-3,6-dioxa-2,7-disilaoctane $(10.0 \mathrm{~mL}, 40.9 \mathrm{mmol})$ in $\mathrm{CH}_{2} \mathrm{Cl}_{2}(60 \mathrm{~mL})$ was added TMSOTf $(0.49 \mathrm{~mL}, 2.73 \mathrm{mmol})$ dropwise at $-15{ }^{\circ} \mathrm{C}$. The mixture was stirred at the same temperature for $4 \mathrm{~h}$, quenched with saturated aqueous $\mathrm{NaHCO}_{3}$ solution $(40 \mathrm{~mL})$, and extracted with $\mathrm{CH}_{2} \mathrm{Cl}_{2}(3 \times 40 \mathrm{~mL})$. The combined organic phase was dried over anhydrous $\mathrm{Na}_{2} \mathrm{SO}_{4}$ and concentrated in vacuo. The residue was chromatographed on silica-gel column with petroleum ether/ethyl acetate (2:1) to give the product $(+)-17(5.20 \mathrm{~g}, 90 \%$ yield $)$ as a white solid: $\mathrm{mp} 45-47^{\circ} \mathrm{C} ; R_{\mathrm{f}} 0.5$ (petroleum ether/ethyl acetate $\left.=1: 1\right) ;[\alpha]_{\mathrm{D}}^{25}+175.8\left(c 1.0, \mathrm{CHCl}_{3}\right)$ IR (KBr): $v_{\max }=2979,2940,2891,1731,1574,1475,1445,1416,1265,1212,1149,1122,1016,993$, $842 \mathrm{~cm}^{-1} ;{ }^{1} \mathrm{H}$ NMR $\left(400 \mathrm{MHz}, \mathrm{CDCl}_{3}\right) \delta: 6.86(\mathrm{~d}, J=2.0 \mathrm{~Hz}, 1 \mathrm{H}), 6.85(\mathrm{~d}, J=2.0 \mathrm{~Hz}, 1 \mathrm{H}), 5.85(\mathrm{dd}, J$ $=9.6,1.6 \mathrm{~Hz}, 1 \mathrm{H}), 5.58(\mathrm{dd}, J=9.6,1.6 \mathrm{~Hz}, 1 \mathrm{H}), 4.54-4.50(\mathrm{~m}, 1 \mathrm{H}), 4.27-4.20(\mathrm{~m}, 1 \mathrm{H}), 4.18-4.12(\mathrm{~m}$, 2H), 4.10-4.03 (m, 2H), 3.96-3.88 (m, 1H), $3.83(\mathrm{~s}, 3 \mathrm{H}), 2.95(\mathrm{~d}, J=14.8 \mathrm{~Hz}, 1 \mathrm{H}), 2.79(\mathrm{~d}, J=14.8$ $\mathrm{Hz}, 1 \mathrm{H}), 2.56(\mathrm{~d}, J=13.2 \mathrm{~Hz}, 1 \mathrm{H}), 2.21$ (ddd, $J=13.2,4.8,2.0 \mathrm{~Hz}, 1 \mathrm{H}), 1.25(\mathrm{t}, J=7.2 \mathrm{~Hz}, 3 \mathrm{H}) ;{ }^{13} \mathrm{C}$ NMR (101 MHz, $\left.\mathrm{CDCl}_{3}\right) \delta: 170.1,149.1,141.2,138.7,129.0,125.6,117.8,113.5,111.5,104.3,74.0$, 65.8, 65.4, 60.7, 56.1, 41.1, 34.5, 30.8, 14.2; $\operatorname{HRMS}(m / z)$ : calcd for $\mathrm{C}_{19} \mathrm{H}_{21} \mathrm{BrNaO}_{6}\left([\mathrm{M}+\mathrm{Na}]^{+}\right)$ 447.0414, found 447.0419 . 


\section{Synthesis of compound $(+)-18$}

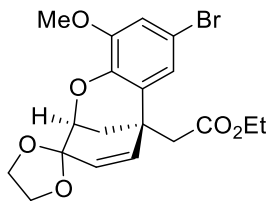

$(+)-17$

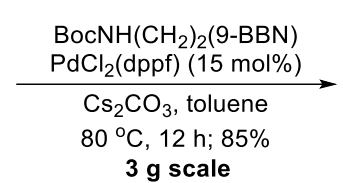

3 g scale

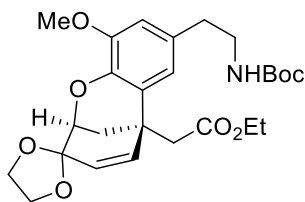

$(+)-18$

The mixture of compound (+)-17 (3.50 g, $8.23 \mathrm{mmol}), \mathrm{PdCl}_{2}(\mathrm{dppf}) \cdot \mathrm{CH}_{2} \mathrm{Cl}_{2}(1.00 \mathrm{~g}, 1.23 \mathrm{mmol})$, and was dissolved in toluene (35 mL). A solution of $\mathrm{Cs}_{2} \mathrm{CO}_{3}(8.04 \mathrm{~g}, 24.7 \mathrm{mmol})$ in $\mathrm{H}_{2} \mathrm{O}(8 \mathrm{~mL})$ and a solution of BocNH$\left(\mathrm{CH}_{2}\right)_{2}(9-\mathrm{BBN})$, which was generated by in situ by the reaction of tert-butyl vinylcarbamate $(1.77 \mathrm{~g}, 12.4 \mathrm{mmol})$ and $(9-B B N)_{2}(1.51 \mathrm{~g}, 6.18 \mathrm{mmol})$ in THF $(12 \mathrm{~mL})$ at room temperature for $4 \mathrm{~h}$, was added. The resulting mixture was stirred at $80{ }^{\circ} \mathrm{C}$ for $12 \mathrm{~h}$, and then filtered through a pad of Florisil. The filtrate was washed with water $(30 \mathrm{~mL})$, and the aqueous phase was extracted with ethyl acetate $(3 \times 50 \mathrm{~mL})$. The combined organic phase was dried over anhydrous $\mathrm{Na}_{2} \mathrm{SO}_{4}$ and concentrated in vacuo. The residue was chromatographed on silica-gel column with petroleum ether/ethyl acetate $(1: 1)$ to give the product $(+)-\mathbf{1 8}(3.43 \mathrm{~g}, 85 \%$ yield $)$ as a light yellow-green solid: $\mathrm{mp} 44-46{ }^{\circ} \mathrm{C} ; R_{\mathrm{f}} 0.45$ (petroleum ether/ethyl acetate $\left.=2: 3\right) ;[\alpha]_{\mathrm{D}}^{25}+142.6(c 1.0$, $\mathrm{CHCl}_{3}$ ); IR (KBr): $v_{\max }=3374,2977,2937,2897,1710,1589,1484,1366,1158,1051,992,950,733$ $\mathrm{cm}^{-1}$; ${ }^{1} \mathrm{H}$ NMR (400 MHz, $\left.\mathrm{CDCl}_{3}\right)$ 8: $6.58(\mathrm{~s}, 1 \mathrm{H}), 6.54(\mathrm{~s}, 1 \mathrm{H}), 5.88(\mathrm{dd}, J=10.0,2.0 \mathrm{~Hz}, 1 \mathrm{H}), 5.56$ (dd, $J=10.0,2.0 \mathrm{~Hz}, 1 \mathrm{H}), 4.55$ (brs, $1 \mathrm{H}), 4.53-4.50(\mathrm{~m}, 1 \mathrm{H}), 4.28-4.20(\mathrm{~m}, 1 \mathrm{H}), 4.19-4.10(\mathrm{~m}, 2 \mathrm{H})$, 4.10-4.01 (m, 2H), 3.97-3.89 (m, 1H), 3.84 (s, 3H), 3.38-3.25 (m, 2H), 3.00 (d, $J=14.8 \mathrm{~Hz}, 1 \mathrm{H}), 2.80$ (d, $J=14.8 \mathrm{~Hz}, 1 \mathrm{H}), 2.69$ (t, $J=6.8 \mathrm{~Hz}, 2 \mathrm{H}), 2.55$ (d, $J=13.2 \mathrm{~Hz}, 1 \mathrm{H}), 2.19$ (ddd, $J=13.2,5.2,2.4 \mathrm{~Hz}$ 1H), 1.44 (s, 9H), 1.24 (t, $J=7.2 \mathrm{~Hz}, 3 \mathrm{H}) ;{ }^{13} \mathrm{C}$ NMR $\left(101 \mathrm{MHz}, \mathrm{CDCl}_{3}\right) \delta: 170.5,155.9,148.2,140.4$, 139.3, 129.8, 127.5, 125.1, 114.9, 110.7, 104.5, 79.2, 73.7, 65.7, 65.4, 60.6, 55.8, 41.9, 41.3, 36.0, 34.5, 31.0, 28.4, 14.2; $\mathrm{HRMS}(\mathrm{m} / \mathrm{z})$ : calcd for $\left.\mathrm{C}_{26} \mathrm{H}_{35} \mathrm{NNaO}_{8}[\mathrm{M}+\mathrm{Na}]^{+}\right)$512.2255, found 512.2258.

\section{Synthesis of compound $(+)-19$}

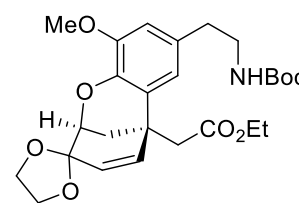

$(+)-18$

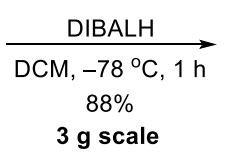

3 g scale

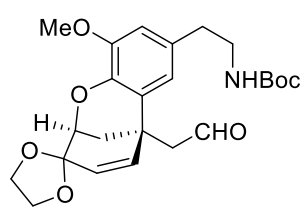

$(+)-19$

To a solution of compound (+)-18 $(3.40 \mathrm{~g}, 6.94 \mathrm{mmol})$ in $\mathrm{CH}_{2} \mathrm{Cl}_{2}(40 \mathrm{~mL})$ at $-78{ }^{\circ} \mathrm{C}$ was added DIBALH (13.9 mL, 1.0 $\mathrm{M}$ in hexane, $13.9 \mathrm{mmol})$ dropwise. The resulting mixture was stirred at $78{ }^{\circ} \mathrm{C}$ for $1 \mathrm{~h}$ and then quenched with saturated aqueous sodium-potassium tartrate solution $(30 \mathrm{~mL})$. The reaction mixture was extracted with $\mathrm{CH}_{2} \mathrm{Cl}_{2}(3 \times 30 \mathrm{~mL})$, dried over anhydrous $\mathrm{Na}_{2} \mathrm{SO}_{4}$ and concentrated in vacuo. The residue was chromatographed on silica-gel column with petroleum ether/ethyl acetate (1:1) to give the product (+)-19 $\left(2.72 \mathrm{~g}, 88 \%\right.$ yield) as a white solid: $\mathrm{mp} 75-77{ }^{\circ} \mathrm{C}$; $R_{\mathrm{f}} 0.34$ (petroleum ether/ethyl acetate $\left.=2: 3\right) ;[\alpha]_{\mathrm{D}}^{25}+155.7\left(c 1.0, \mathrm{CHCl}_{3}\right) ; \mathrm{IR}(\mathrm{KBr}): v_{\max }=3599,3354$, 2966, 2932, 2897, 1708, 1589, 1516, 1483, 1366, 1273, 1166, 1144, 950, 844, $733 \mathrm{~cm}^{-1} ;{ }^{1} \mathrm{H}$ NMR (400 $\left.\mathrm{MHz}, \mathrm{CDCl}_{3}\right) \delta: 9.82(\mathrm{~s}, 1 \mathrm{H}), 6.61(\mathrm{~s}, 1 \mathrm{H}), 6.50(\mathrm{~s}, 1 \mathrm{H}), 5.90(\mathrm{dd}, J=10.0,1.6 \mathrm{~Hz}, 1 \mathrm{H}), 5.62(\mathrm{dd}, J=$ 10.0, $1.6 \mathrm{~Hz}, 1 \mathrm{H}), 4.59-4.48(\mathrm{~m}, 2 \mathrm{H}), 4.31-4.23(\mathrm{~m}, 1 \mathrm{H}), 4.14-4.03(\mathrm{~m}, 2 \mathrm{H}), 3.97-3.91(\mathrm{~m}, 1 \mathrm{H}), 3.85$ (s, 3H), 3.33-3.25 (m, 2H), 2.97-2.91 (m, 2H), $2.70(\mathrm{t}, J=6.8 \mathrm{~Hz}, 2 \mathrm{H}), 2.47$ (d, $J=13.2 \mathrm{~Hz}, 1 \mathrm{H}), 2.18$ (ddd, $J=13.2,4.8,2.0 \mathrm{~Hz}, 1 \mathrm{H}), 1.44$ (s, 9H); ${ }^{13} \mathrm{C}$ NMR $\left(101 \mathrm{MHz}, \mathrm{CDCl}_{3}\right) \delta: 200.6,155.9,148.5$, 140.4, 138.7, 130.3, 126.8, 126.0, 115.0, 110.9, 104.3, 79.3, 73.5, 65.8, 65.4, 55.9, 49.3, 41.9, 36.0, 


\section{Synthesis of compound $(+)-20$}

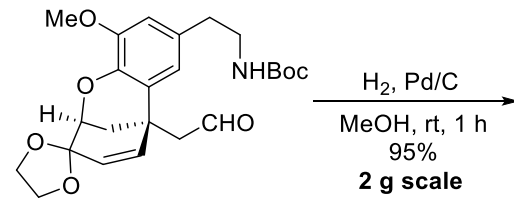

$(+)-19$

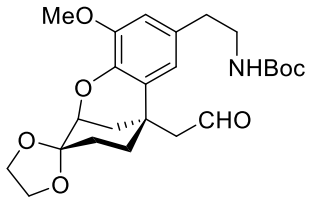

$(+)-20$

The mixture of compound $(+)-19(2.67 \mathrm{~g}, 6.00 \mathrm{mmol})$ and $10 \% \mathrm{Pd} / \mathrm{C}(0.27 \mathrm{~g})$ in $\mathrm{MeOH}(30 \mathrm{~mL})$ was charged with $\mathrm{H}_{2}$ balloon, and the mixture was stirred at room temperature for $1 \mathrm{~h}$. The mixture was then filtered through a pad of Florisil and concentrated in vacuo. The residue was chromatographed on silica-gel column with petroleum ether/ethyl acetate (1:1) to give the product $(+)-20(2.57 \mathrm{~g}, 95 \%$ yield $)$ as a white solid: $\mathrm{mp} 123-125{ }^{\circ} \mathrm{C} ; R_{\mathrm{f}} 0.55$ (petroleum ether/ethyl acetate, $\left.1: 2\right) ;[\alpha]_{\mathrm{D}}^{25}+25.2(c 1.0$, $\left.\mathrm{CHCl}_{3}\right)$; IR (KBr): $v_{\max }=3727,3703,3626,3602,3369,2934,2890,1714,1588,1517,1482,1365$, 1272, 1220, 1169, 1146, 1110, 1037, $733 \mathrm{~cm}^{-1} ;{ }^{1} \mathrm{H}$ NMR $\left(400 \mathrm{MHz}, \mathrm{CDCl}_{3}\right) \delta: 9.66(\mathrm{t}, J=2.8 \mathrm{~Hz}, 1 \mathrm{H})$, $6.61(\mathrm{~s}, 1 \mathrm{H}), 6.55(\mathrm{~s}, 1 \mathrm{H}), 4.64(\mathrm{brs}, 1 \mathrm{H}), 4.33(\mathrm{t}, J=2.8 \mathrm{~Hz}, 1 \mathrm{H}), 4.09-3.99(\mathrm{~m}, 3 \mathrm{H}), 3.95-3.90(\mathrm{~m}$, $1 \mathrm{H}), 3.86(\mathrm{~s}, 3 \mathrm{H}), 3.39-3.21(\mathrm{~m}, 2 \mathrm{H}), 3.02(\mathrm{dd}, J=16.0,2.0 \mathrm{~Hz}, 1 \mathrm{H}), 2.75-2.66(\mathrm{~m}, 2 \mathrm{H}), 2.60(\mathrm{dd}, J=$ 16.0, $2.4 \mathrm{~Hz}, 1 \mathrm{H}), 2.16-2.13(\mathrm{~m}, 2 \mathrm{H}), 1.95-1.85(\mathrm{~m}, 1 \mathrm{H}), 1.66-1.61(\mathrm{~m}, 1 \mathrm{H}), 1.61-1.53(\mathrm{~m}, 2 \mathrm{H}), 1.44$ (s, 9H); ${ }^{13} \mathrm{C}$ NMR (101 MHz, $\left.\mathrm{CDCl}_{3}\right) \delta: 201.5,155.9,148.1,141.9,130.5,126.2,116.6,110.5,107.8$, 79.2, 73.3, 65.0 (2), 55.8, 51.4, 41.9, 38.2, 36.0, 33.1, 31.7, 28.6, 28.4; HRMS $(\mathrm{m} / \mathrm{z})$ : calcd for $\mathrm{C}_{24} \mathrm{H}_{34} \mathrm{NO}_{7}^{+}\left([\mathrm{M}+\mathrm{H}]^{+}\right) 448.2330$, found 448.2327 .

\section{Synthesis of compound (+)-21}

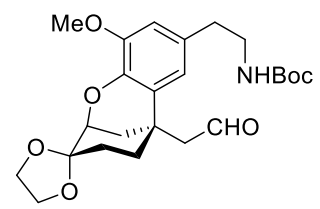

$(+)-20$

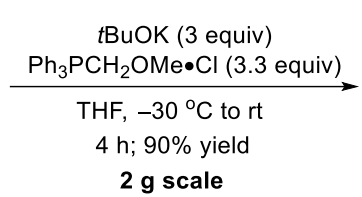

2 g scale

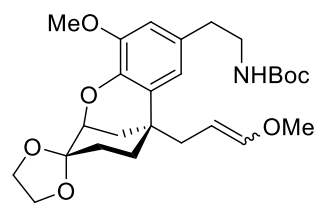

$(+)-21$

To a solution of (methoxymethyl)triphenylphosphonium chloride ( $6.86 \mathrm{~g}, 20.0 \mathrm{mmol})$ in THF (20 $\mathrm{mL})$ was added $t \mathrm{BuOK}(2.08 \mathrm{~g}, 18.0 \mathrm{mmol})$ in portions at $-30^{\circ} \mathrm{C}$, and the resulting suspension solution was stirred at the same temperature for $0.5 \mathrm{~h}$. The solution of compound (+)-20 $(2.69 \mathrm{~g}, 6.00 \mathrm{mmol})$ in $15 \mathrm{~mL}$ THF was then added, and the resulting mixture was stirred at room temperature for another $4 \mathrm{~h}$. The reaction mixture was quenched with $\mathrm{H}_{2} \mathrm{O}(30 \mathrm{~mL})$ and extracted with ethyl acetate $(4 \times 30 \mathrm{~mL})$. The combined organic phase was washed with brine, dried over anhydrous $\mathrm{Na}_{2} \mathrm{SO}_{4}$, and concentrated in vacuo. The residue was chromatographed on silica-gel column with petroleum ether/ethyl acetate (2:1 to 1:1) to give the product (+)-21 as a mixture of $Z$ and $E$ isomers (44:56, $2.57 \mathrm{~g}, 90 \%$ yield): $\mathrm{mp}$ $46-51{ }^{\circ} \mathrm{C} ; R_{\mathrm{f}} 0.53$ (petroleum ether/ethyl acetate $\left.=1: 1\right) ;[\alpha]_{\mathrm{D}}^{25}+33.8\left(c 1.0, \mathrm{CHCl}_{3}\right) ; \mathrm{IR}(\mathrm{KBr}): v_{\max }=$ $3632,3359,2932,1708,1587,1516,1480,1365,1250,1145,1022,949,733 \mathrm{~cm}^{-1} ;{ }^{1} \mathrm{H}$ NMR (400 $\left.\mathrm{MHz}, \mathrm{CDCl}_{3}\right) \delta: 6.62(\mathrm{~s}, 0.44 \mathrm{H}), 6.60(\mathrm{~s}, 0.56 \mathrm{H}), 6.56(\mathrm{~s}, 1 \mathrm{H}), 6.30(\mathrm{~d}, J=12.8 \mathrm{~Hz}, 0.56 \mathrm{H}), 5.93(\mathrm{~d}, J$ $=6.4 \mathrm{~Hz}, 0.44 \mathrm{H}), 4.65-4.54(\mathrm{~m}, 1.56 \mathrm{H}), 4.30(\mathrm{~m}, 1.2 \mathrm{H}), 4.24(\mathrm{q}, J=7.2 \mathrm{~Hz}, 0.56 \mathrm{H}), 4.09-3.95(\mathrm{~m}$, $3.7 \mathrm{H}), 3.95-3.86(\mathrm{~m}, 1.0 \mathrm{H}), 3.85(\mathrm{~s}, 1.68 \mathrm{H}), 3.84(\mathrm{~s}, 1.32 \mathrm{H}), 3.59(\mathrm{~s}, 1.32 \mathrm{H}), 3.45(\mathrm{~s}, 1.56 \mathrm{H})$, $3.37-3.25(\mathrm{~m}, 2.0 \mathrm{H}), 2.75-2.66(\mathrm{~m}, 2.0 \mathrm{H}), 2.60-2.43(\mathrm{~m}, 1.44 \mathrm{H}), 2.22(\mathrm{dd}, J=14.0,8.8 \mathrm{~Hz}, 0.56 \mathrm{H})$, 1.96-1.75 (m, 4H), 1.57-1.50 (m, 2H), $1.44(\mathrm{~s}, 9 \mathrm{H}) ;{ }^{13} \mathrm{C}$ NMR (101 MHz, $\left.\mathrm{CDCl}_{3}\right)$ 8: 155.9, 149.0, 147.8, 147.7, 142.1, 129.8, 129.6, 128.1 (2), 117.2, 116.8, 109.8 (2), 108.4 (2), 101.7, 97.8, 79.1, 73.7 
(2), 65.0, 64.9, 59.4 (2), 56.0, 55.8, 42.1, 42.0, 37.9, 37.7, 36.9, 36.0, 35.9, 34.3, 34.1, 32.7, 30.8, 29.0, 28.4; HRMS $(m / z)$ : calcd for $\mathrm{C}_{26} \mathrm{H}_{41} \mathrm{~N}_{2} \mathrm{O}_{7}\left(\left[\mathrm{M}+\mathrm{NH}_{4}\right]^{+}\right)$493.2908, found 493.2905.

\section{Synthesis of compound $(+)-10$}

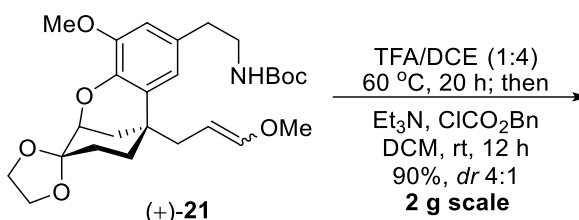

$(+)-21$

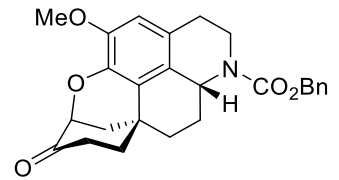

$(+)-10$

To a solution of (+)-21 $(2.80 \mathrm{~g}, 5.89 \mathrm{mmol})$ in DCE $(55 \mathrm{~mL})$ was added $14 \mathrm{~mL}$ TFA dropwise. The resulting reaction mixture was stirred at $60{ }^{\circ} \mathrm{C}$ for $20 \mathrm{~h}$ and then concentrated in vacuo. The residue was re-dissolved with $\mathrm{CH}_{2} \mathrm{Cl}_{2}(40 \mathrm{~mL})$, and $\mathrm{Et}_{3} \mathrm{~N}(6.09 \mathrm{~mL}, 44.0 \mathrm{mmol})$ and $\mathrm{ClCO}_{2} \mathrm{Bn}(3.43 \mathrm{~mL}, 23.6$ $\mathrm{mmol}$ ) were added dropwise successively at room temperature. The reaction mixture was stirred for 12 h, quenched with $\mathrm{H}_{2} \mathrm{O}(40 \mathrm{~mL})$, and extracted with $\mathrm{CH}_{2} \mathrm{Cl}_{2}(3 \times 30 \mathrm{~mL})$. The combined organic phase was dried over anhydrous $\mathrm{Na}_{2} \mathrm{SO}_{4}$ and concentrated in vacuo. The residue was chromatographed on silica-gel column with petroleum ether/ethyl acetate (1:1) to give the product $(+)-\mathbf{1 0}$ as a mixture of two diastereoisomers $\left(d r=4: 1,2.30 \mathrm{~g}, 90 \%\right.$ yield): white solid, mp $62-67{ }^{\circ} \mathrm{C} ; R_{\mathrm{f}} 0.46$ (petroleum ether/ethyl acetate $=1: 1) ;[\alpha]_{\mathrm{D}}^{25}+149.0\left(c 1.0, \mathrm{CHCl}_{3}\right) ; \mathrm{IR}(\mathrm{KBr}): v_{\max }=2938,2866,1725,1695,1600$, 1486, 1423, 1280, 1195, 1101, 990, 735, $700 \mathrm{~cm}^{-1} ;{ }^{1} \mathrm{H}$ NMR (400 MHz, $\left.\mathrm{CDCl}_{3}\right) \delta: 7.53-7.28(\mathrm{~m}, 5 \mathrm{H})$, $6.61(\mathrm{~s}, 1 \mathrm{H}), 5.27-5.18(\mathrm{~m}, 2 \mathrm{H}), 4.94-4.82(\mathrm{~m}, 0.85 \mathrm{H}), 4.75(\mathrm{~d}, J=4.8 \mathrm{~Hz}, 0.15 \mathrm{H}), 4.71-4.69(\mathrm{~m}$, $0.85 \mathrm{H}), 4.58-4.56(\mathrm{~m}, 1.5 \mathrm{H}), 4.48-4.39(\mathrm{~m}, 1 \mathrm{H}), 3.87(\mathrm{~s}, 3 \mathrm{H}), 2.99-2.76(\mathrm{~m}, 2 \mathrm{H}), 2.65-2.56(\mathrm{~m}, 1 \mathrm{H})$, 2.47-2.18 (m, 4H), 2.15-2.03 (m, 2H), 1.97-1.83 (m, 2H), $1.73(\mathrm{~m}, 1 \mathrm{H}), 1.64-1.49(\mathrm{~m}, 1 \mathrm{H}) ;{ }^{13} \mathrm{C}$ NMR $\left(101 \mathrm{MHz}, \mathrm{CDCl}_{3}\right) \delta: 206.0,155.2,146.4,140.2,136.8,128.5,128.0,127.8,127.8,125.9,123.7,110.2$, 78.5, 67.1, 56.0, 49.4, 40.2, 39.2, 39.1, 36.6, 32.2, 31.2, 30.7, 29.9; HRMS (m/z): calcd for $\mathrm{C}_{26} \mathrm{H}_{28} \mathrm{NO}_{5}$ $\left([\mathrm{M}+\mathrm{H}]^{+}\right)$434.1962, found 434.1957. 


\section{D) Enantioselective Synthesis of Pentacyclic Homoproaporphine Alkaloids}

The advanced chiral intermediate (-)-9 and the members of pentacyclic homoproaphine alkaloid family were synthesized by the following procedure.
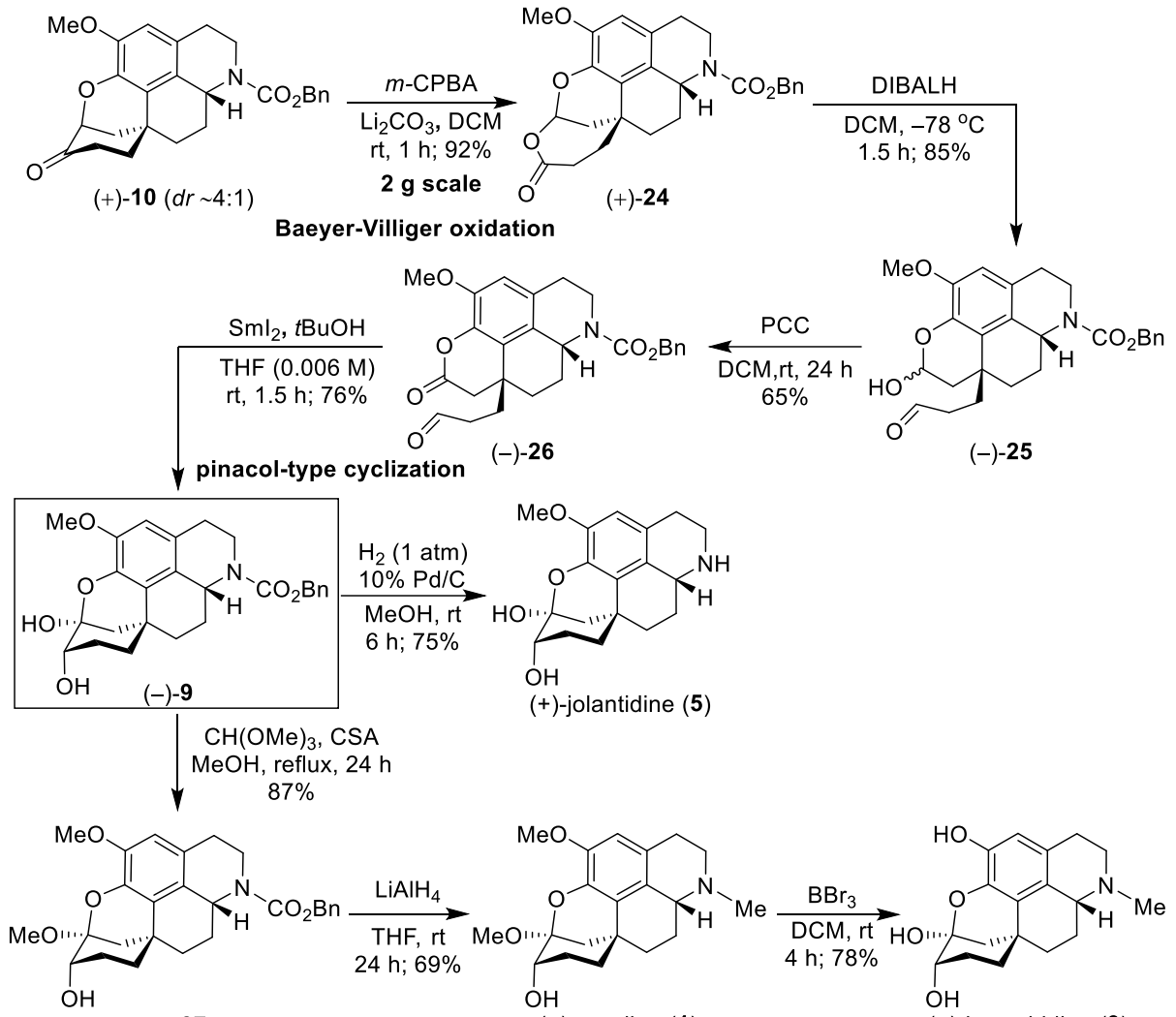

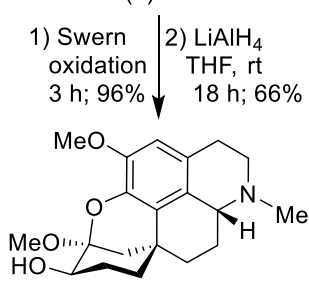

$(+)$-regelinine $(6)$ $(+)$-regeline (1)

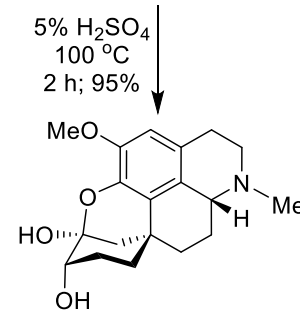

$(+)$-regalamine (2) $(+)$-kesselridine (3)

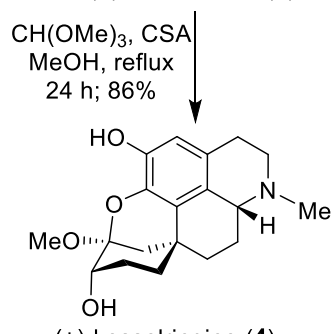

\section{Synthesis of compound $(+)-24$}

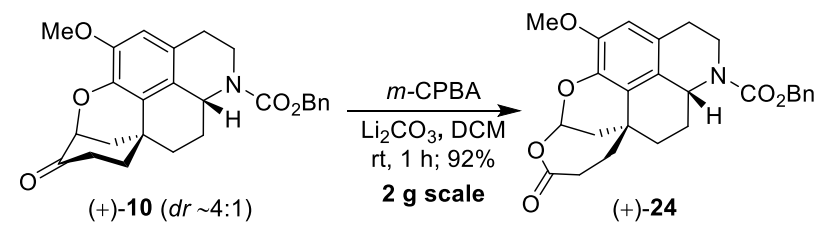

To a solution of compound (+)-10 (2.30 g, $5.31 \mathrm{mmol})$ in $\mathrm{CH}_{2} \mathrm{Cl}_{2}(35 \mathrm{~mL})$ was added $\mathrm{Li}_{2} \mathrm{CO}_{3}$ $(0.12 \mathrm{~g}, 1.60 \mathrm{mmol})$ and $m$-CPBA $(1.47 \mathrm{~g}, 6.80 \mathrm{mmol})$. The resulting mixture was stirred at room temperature for $1 \mathrm{~h}$, and quenched with saturated $\mathrm{Na}_{2} \mathrm{CO}_{3}$ solution $(30 \mathrm{~mL})$, and extracted with $\mathrm{CH}_{2} \mathrm{Cl}_{2}$ $(3 \times 40 \mathrm{~mL})$. The combined organic phase was dried over anhydrous $\mathrm{Na}_{2} \mathrm{SO}_{4}$ and concentrated in vacuo. The residue was chromatographed on silica-gel column with petroleum ether/ethyl acetate (2:1) to give the product $(+)-24\left(2.20 \mathrm{~g}, 92 \%\right.$ yield) as a white solid, $\mathrm{mp} 83-85{ }^{\circ} \mathrm{C} ; R_{\mathrm{f}} 0.47$ (petroleum ether/ethyl acetate =1:1); $[\alpha]_{\mathrm{D}}^{25}+55.8\left(c 1.0, \mathrm{CHCl}_{3}\right) ; \mathrm{IR}(\mathrm{KBr}): v_{\max }=2930,2871,1740,1695,1423,1266,1099$, 1036, 737, $701 \mathrm{~cm}^{-1}$; ${ }^{1} \mathrm{H}$ NMR (400 MHz, $\mathrm{CDCl}_{3}$ ) $\delta: 7.45-7.29(\mathrm{~m}, 5 \mathrm{H}), 6.64(\mathrm{~s}, 1 \mathrm{H}), 5.97(\mathrm{dd}, J=5.2$, 
$0.8 \mathrm{~Hz}, 1 \mathrm{H}), 5.30-5.14(\mathrm{~m}, 2 \mathrm{H}), 4.76(\mathrm{dd}, J=12.0,4.4 \mathrm{~Hz}, 1 \mathrm{H}), 4.47-4.37(\mathrm{~m}, 1 \mathrm{H}), 3.88(\mathrm{~s}, 3 \mathrm{H}), 2.93-$ $2.80(\mathrm{~m}, 2 \mathrm{H}), 2.66-2.58(\mathrm{~m}, 1 \mathrm{H}), 2.57-2.49(\mathrm{~m}, 1 \mathrm{H}), 2.48-2.37(\mathrm{~m}, 1 \mathrm{H}), 2.34-2.20(\mathrm{~m}, 2 \mathrm{H}), 2.16-2.08$ $(\mathrm{m}, 1 \mathrm{H}), 2.03-1.95(\mathrm{~m}, 1 \mathrm{H}), 1.93-1.83(\mathrm{~m}, 1 \mathrm{H}), 1.79-1.70(\mathrm{~m}, 1 \mathrm{H}), 1.61-1.48(\mathrm{~m}, 1 \mathrm{H}) ;{ }^{13} \mathrm{C} \mathrm{NMR}(101$ $\left.\mathrm{MHz}, \mathrm{CDCl}_{3}\right) \delta: 172.8,155.1,145.9,140.3,136.8,128.6,128.1,127.8,127.4,125.1,123.3,110.6$, $94.7,67.2,56.1(2), 49.8,40.9,39.1,36.1,35.5,33.2,32.4,31.9,30.1$; HRMS $(\mathrm{m} / \mathrm{z})$ : calcd for $\mathrm{C}_{26} \mathrm{H}_{28} \mathrm{NO}_{6}\left([\mathrm{M}+\mathrm{H}]^{+}\right) 450.1911$, found 450.1904 .

Synthesis of compound (-)-25
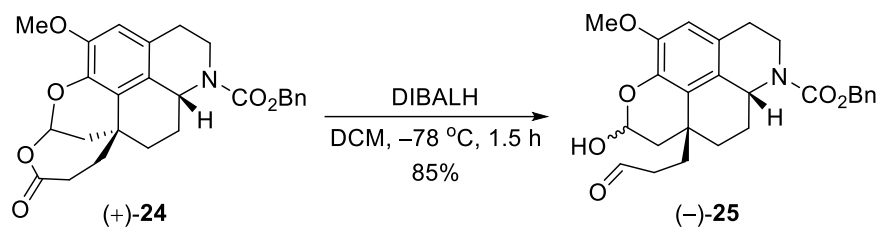

To a solution of compound (+)-24 $(0.66 \mathrm{~g}, 1.47 \mathrm{mmol})$ in $\mathrm{CH}_{2} \mathrm{Cl}_{2}(15 \mathrm{~mL})$ at $-78{ }^{\circ} \mathrm{C}$ was added DIBALH (2.20 mL, 1.0 M in hexane, $2.20 \mathrm{mmol})$ dropwise. The mixture was stirred at $-78{ }^{\circ} \mathrm{C}$ for 1.5 $\mathrm{h}$, quenched with saturated aqueous solution of sodium-potassium tartrate $(20 \mathrm{~mL})$, and extracted with $\mathrm{CH}_{2} \mathrm{Cl}_{2}(3 \times 20 \mathrm{~mL})$. The combined organic phase was dried over anhydrous $\mathrm{Na}_{2} \mathrm{SO}_{4}$ and concentrated in vacuo. The residue was chromatographed on silica-gel column with petroleum ether/ethyl acetate (1:1 to $100 \%$ ethyl acetate) to give the product (-)-25 (0.56 g, 85\% yield) as a mixture of diastereoisomers (not stable). $R_{\mathrm{f}} 0.58\left(100 \%\right.$ ethyl acetate); $[\alpha]_{\mathrm{D}}^{25}-123.0\left(c 1.0, \mathrm{CHCl}_{3}\right)$; IR $(\mathrm{KBr}): v_{\max }$ $=3744,3479,2930,1695,1424,1358,1269,1195,1101,735,699 \mathrm{~cm}^{-1} ;{ }^{1} \mathrm{H} \mathrm{NMR}\left(400 \mathrm{MHz}, \mathrm{CDCl}_{3}\right)$

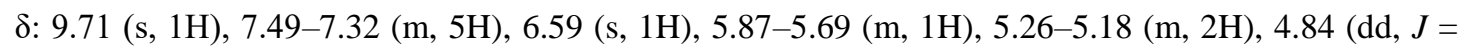
10.4, 5.6 Hz, 1H), 4.46-4.35 (m, 1H), 3.86 (s, 3H), 2.90-2.83 (m, 2H), 2.64-2.56 (m, 1H), 2.53-2.45 (m, 1H), 2.44-2.26 (m, 2H), $2.22(\mathrm{dd}, J=13.9,4.0 \mathrm{~Hz}, 1 \mathrm{H}), 2.16-2.05(\mathrm{~m}, 1 \mathrm{H}), 1.97-1.84(\mathrm{~m}, 2 \mathrm{H})$, $1.68(\mathrm{dd}, J=13.9,8.8 \mathrm{~Hz}, 2 \mathrm{H}), 1.55-1.48(\mathrm{~m}, 1 \mathrm{H}) ;{ }^{13} \mathrm{C} \mathrm{NMR}\left(101 \mathrm{MHz}, \mathrm{CDCl}_{3}\right) \delta: 201.2,155.3,147.1$, 139.9, 136.8, 128.6, 128.5, 128.1, 128.0, 127.8, 126.8, 110.3, 92.7, 67.2, 55.9, 42.1, 39.8, 39.5, 39.2, 34.6, 33.0, 32.4, 30.4, 29.3; HRMS $(\mathrm{m} / z)$ : calcd for $\mathrm{C}_{26} \mathrm{H}_{30} \mathrm{NO}_{6}\left([\mathrm{M}+\mathrm{H}]^{+}\right)$452.2068, found 452.2074.

\section{Synthesis of compound (-)-26}
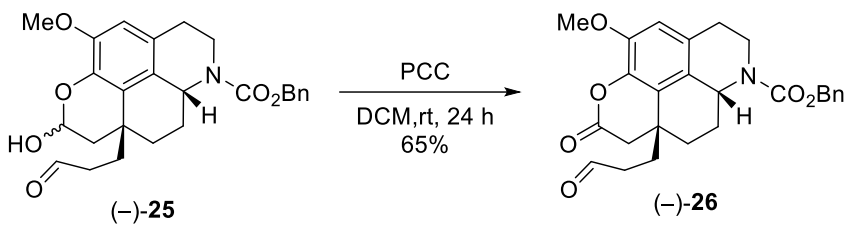

To a solution of compound (-)-25 $(0.35 \mathrm{~g}, 0.77 \mathrm{mmol})$ in $\mathrm{CH}_{2} \mathrm{Cl}_{2}(15 \mathrm{~mL})$ was added pyridinium chlorochromate (PCC, $0.26 \mathrm{~g}, 1.20 \mathrm{mmol}$ ), and the mixture was stirred at room temperature for $1.5 \mathrm{~h}$. The reaction mixture was filtrated through a short plug of silica gel and concentrated in vacuo. The residue was chromatographed on silica-gel column with petroleum ether/ethyl acetate (1:1) to give the product (-)-26 (0.23 g, 65\% yield) as a white solid (with a minor diastereoisomer): $\mathrm{mp} 59-62{ }^{\circ} \mathrm{C} ; R_{\mathrm{f}}$ 0.4 (petroleum ether/ethyl acetate $=1: 1) ;[\alpha]_{\mathrm{D}}^{25}-36.6\left(c 1.0, \mathrm{CHCl}_{3}\right) ; \mathrm{IR}(\mathrm{KBr}): v_{\max }=3733,3625,2939$, 1769, 1696, 1488,1447, 1268, 1229, 1195, 1126,1100, 904, 737, $700 \mathrm{~cm}^{-1} ;{ }^{1} \mathrm{H} \mathrm{NMR}\left(400 \mathrm{MHz}, \mathrm{CDCl}_{3}\right)$

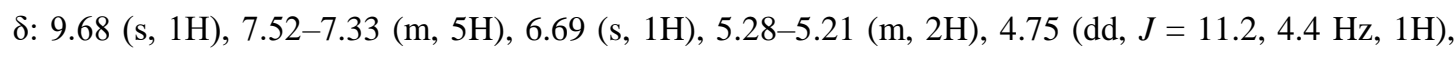
4.47-4.45 (m, 1H), $3.90(\mathrm{~s}, 3 \mathrm{H}), 2.94-2.82(\mathrm{~m}, 2 \mathrm{H}), 2.77(\mathrm{~d}, J=16.0 \mathrm{~Hz}, 1 \mathrm{H}), 2.71-2.59(\mathrm{~m}, 2 \mathrm{H})$, 2.55-2.46 (m, 1H), 2.45-2.28 (m, 2H), 2.05-1.99 (m, 1H), 1.99-1.89 (m, 2H), 1.75-1.68 (m, 1H), 
$1.57-1.48(\mathrm{~m}, 1 \mathrm{H}) ;{ }^{13} \mathrm{C}$ NMR $\left(101 \mathrm{MHz}, \mathrm{CDCl}_{3}\right) \delta: 200.1,167.0,155.2,146.3,138.8,136.6,130.8$, 128.6, 128.1, 128.1, 127.9, 125.7, 125.1, 111.4, 67.3, 56.1, 50.5, 42.3, 39.2, 38.9, 35.1, 32.5, 31.7, 30.6; HRMS $(m / z)$ : calcd for $\mathrm{C}_{26} \mathrm{H}_{28} \mathrm{NO}_{6}\left([\mathrm{M}+\mathrm{H}]^{+}\right) 450.1911$, found 450.1916 .

Synthesis of compound (-)-9

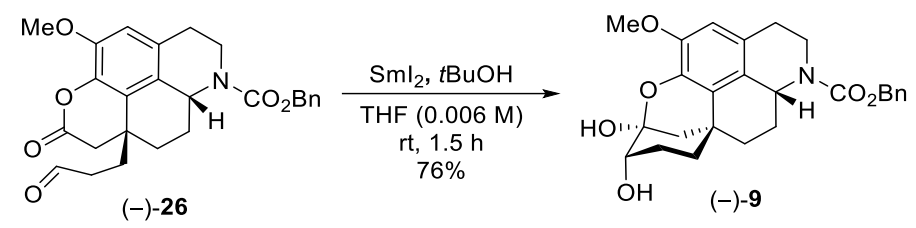

To a solution of compound (-)-26 $(0.23 \mathrm{~g}, 0.50 \mathrm{mmol})$ in THF $(83 \mathrm{~mL})$ were added $t \mathrm{BuOH}(0.23$ $\mathrm{mL}, 2.50 \mathrm{mmol})$ and $\mathrm{SmI}_{2}(25.0 \mathrm{~mL}, 2.50 \mathrm{mmol}, 0.1 \mathrm{M}$ in THF) at room temperature. The mixture was stirred at room temperature for $1.5 \mathrm{~h}$, quenched with saturated aqueous $\mathrm{NH}_{4} \mathrm{Cl}$ solution $(50 \mathrm{~mL})$, and extracted with $\mathrm{Et}_{2} \mathrm{O}(3 \times 50 \mathrm{~mL})$. The combined organic phase was dried over anhydrous $\mathrm{Na}_{2} \mathrm{SO}_{4}$ and concentrated in vacuo. The residue was chromatographed on silica-gel column with petroleum ether/ethyl acetate (1:1 to $100 \%$ ethyl acetate) to give the product (-)-9 $(0.17 \mathrm{~g}, 76 \%$ yield $)$ as a white solid: mp 98-100 ${ }^{\circ} \mathrm{C} ; R_{\mathrm{f}} 0.41$ (100\% ethyl acetate); $[\alpha]_{\mathrm{D}}^{25}-86.4\left(c 1.0, \mathrm{CHCl}_{3}\right)$; IR $(\mathrm{KBr}): v_{\max }=3463$, 2930, 2866, 1695, 1601, 1427, 1358, 1267, 1173, 1095, 1053, 736, $700 \mathrm{~cm}^{-1}$; ${ }^{1} \mathrm{H}$ NMR (400 MHz, $\left.\mathrm{CD}_{3} \mathrm{OD}\right) \delta: 7.48-7.21(\mathrm{~m}, 5 \mathrm{H}), 6.60(\mathrm{~s}, 1 \mathrm{H}), 5.22-5.12(\mathrm{~m}, 2 \mathrm{H}), 4.71(\mathrm{dd}, J=11.6,4.8 \mathrm{~Hz}, 1 \mathrm{H}), 4.37-$ $4.23(\mathrm{~m}, 1 \mathrm{H}), 3.76(\mathrm{~s}, 3 \mathrm{H}), 3.73-3.67(\mathrm{~m}, 1 \mathrm{H}), 2.84(\mathrm{t}, J=12.0 \mathrm{~Hz}, 1 \mathrm{H}), 2.70(\mathrm{td}, J=12.0,4.0 \mathrm{~Hz}, 1 \mathrm{H})$, $2.55(\mathrm{~d}, J=15.2 \mathrm{~Hz}, 1 \mathrm{H}), 2.21-2.14(\mathrm{~m}, 2 \mathrm{H}), 1.90-1.82(\mathrm{~m}, 1 \mathrm{H}), 1.72(\mathrm{td}, J=13.2,3.6 \mathrm{~Hz}, 1 \mathrm{H}), 1.66-$ $1.58(\mathrm{~m}, 2 \mathrm{H}), 1.57-1.43(\mathrm{~m}, 3 \mathrm{H}), 1.35(\mathrm{tt}, J=14.4,4.0 \mathrm{~Hz}, 1 \mathrm{H}) ;{ }^{13} \mathrm{C}$ NMR $\left(101 \mathrm{MHz}, \mathrm{CD}_{3} \mathrm{OD}\right) \delta$ : $155.5,145.7,142.5,136.8,127.8,127.5,127.5,124.8,124.5,123.9,110.3,99.3,70.6,66.9,55.1$, 49.7, 38.9, 38.7, 34.6, 33.4, 32.9, 29.2, 27.1; HRMS $(m / z)$ : calcd for $\mathrm{C}_{26} \mathrm{H}_{30} \mathrm{NO}_{6}\left([\mathrm{M}+\mathrm{H}]^{+}\right) 452.2068$, found 452.2065 .

\section{Synthesis of compound (-)-27}

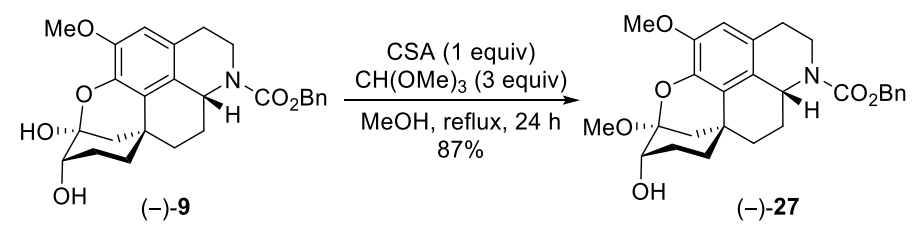

To a solution of compound (-)-9 $(0.19 \mathrm{~g}, 0.42 \mathrm{mmol})$ in $\mathrm{MeOH}(10 \mathrm{~mL})$ were added $\mathrm{CH}(\mathrm{OMe})_{3}$ $(0.14 \mathrm{~mL}, 1.25 \mathrm{mmol})$ and (+)-10-camphorsulfonic acid (CSA, $0.10 \mathrm{~g}, 0.42 \mathrm{mmol})$. The reaction mixture was heated to reflux for $24 \mathrm{~h}$, diluted with saturated aqueous solution of $\mathrm{NaHCO}_{3}(10 \mathrm{~mL})$, and extracted with EtOAc $(3 \times 10 \mathrm{~mL})$. The combined organic phase was dried over anhydrous $\mathrm{Na}_{2} \mathrm{SO}_{4}$ and concentrated in vacuo. The residue was chromatographed on silica-gel column with petroleum ether/ethyl acetate (1:1) to give the product (-)-27 $(0.17 \mathrm{~g}, 87 \%$ yield) as a white solid (with a minor diastereoisomer), mp $75-77{ }^{\circ} \mathrm{C} ; R_{\mathrm{f}} 0.36$ (petroleum ether/ethyl acetate $\left.=1: 1\right) ;[\alpha]_{\mathrm{D}}^{25}-35.5(c \quad 1.0$, $\mathrm{CHCl}_{3}$ ); IR (KBr): $v_{\max }=3483,2938,2838,1695,1425,1267,1198,1097,1061,736 \mathrm{~cm}^{-1} ;{ }^{1} \mathrm{H} \mathrm{NMR}$ $\left(400 \mathrm{MHz}, \mathrm{CDCl}_{3}\right) \delta: 7.41-7.29(\mathrm{~m}, 5 \mathrm{H}), 6.56(\mathrm{~s}, 1 \mathrm{H}), 5.23-5.18(\mathrm{~m}, 2 \mathrm{H}), 4.81(\mathrm{dd}, J=11.2,5.2 \mathrm{~Hz}$, $0.8 \mathrm{H}), 4.53(\mathrm{dd}, J=10.4,5.6 \mathrm{~Hz}, 0.2 \mathrm{H}), 4.48-4.32(\mathrm{~m}, 1 \mathrm{H}), 3.80-3.88(\mathrm{~m}, 4 \mathrm{H}), 3.43(\mathrm{~s}, 3 \mathrm{H}), 2.97-$ $2.77(\mathrm{~m}, 2 \mathrm{H}), 2.57(\mathrm{~d}, J=14.0 \mathrm{~Hz}, 1 \mathrm{H}), 2.43(\mathrm{~s}, 1 \mathrm{H}), 2.34-2.28(\mathrm{~m}, 1 \mathrm{H}), 2.07-2.01(\mathrm{~m}, 1 \mathrm{H}), 1.99-1.82$ $(\mathrm{m}, 2 \mathrm{H}), 1.78-1.63(\mathrm{~m}, 4 \mathrm{H}), 1.44-1.38(\mathrm{~m}, 1 \mathrm{H}) ;{ }^{13} \mathrm{C} \mathrm{NMR}\left(101 \mathrm{MHz}, \mathrm{CDCl}_{3}\right.$, rotamer mixture) $\delta$ : 
156.4, 146.0, 142.5, 136.9, 128.5, 128.0, 127.8, 124.3, 124.3, 122.4, 110.2, 102.2, 71.3, 70.6, 67.0, 56.0, 49.2, 39.1, 35.4, 34.9, 34.6, 33.7, 33.0, 29.9, 26.6; HRMS $(m / z)$ : calcd for $\mathrm{C}_{27} \mathrm{H}_{32} \mathrm{NO}_{6}\left([\mathrm{M}+\mathrm{H}]^{+}\right)$ 466.2224, found 466.2222 .

\section{Synthesis of (+)-regeline (1)}

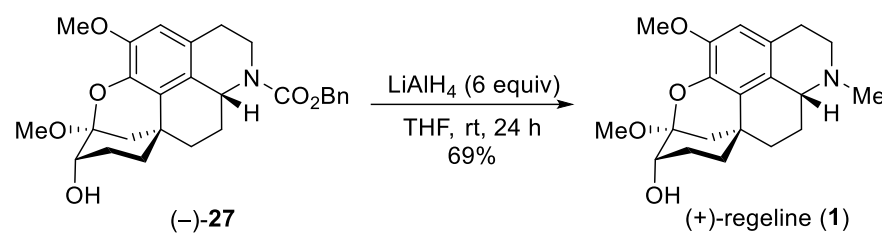

To a solution of $(-)-27(87.0 \mathrm{mg}, 0.19 \mathrm{mmol})$ in THF $(6 \mathrm{~mL})$ was added $\mathrm{LiAlH}_{4}(43.0 \mathrm{mg}, 1.12$ $\mathrm{mmol}$ ), and the resulting mixture was stirred at room temperature for $24 \mathrm{~h}$. The reaction was then quenched by slow addition of $\mathrm{H}_{2} \mathrm{O}(8 \mathrm{~mL})$, and the mixture was extracted with EtOAc $(3 \times 6 \mathrm{~mL})$. The combined organic phase was dried over anhydrous $\mathrm{Na}_{2} \mathrm{SO}_{4}$ and concentrated in vacuo. The residue was chromatographed on silica-gel column with ethyl acetate/triethylamine (30:1) to give the product (+)-regeline (1) $\left(45.0 \mathrm{mg}, 69 \%\right.$ yield) as a white solid: $\mathrm{mp} 201-203{ }^{\circ} \mathrm{C}$ (lit. $\left.{ }^{2} 198-200{ }^{\circ} \mathrm{C}\right) ; R_{\mathrm{f}} 0.26$ (ethyl acetate/triethylamine $=30: 1) ;[\alpha]_{\mathrm{D}}^{25}+92.0(c 1.5, \mathrm{MeOH})\left(\right.$ lit. $^{2}+93(c$ 1.5, MeOH$\left.)\right) ; \mathrm{IR}(\mathrm{KBr})$ : $v_{\max }=3175,2945,2877,2833,1598,1485,1463,1374,1259,1225,1159,1061,1004,872,700 \mathrm{~cm}^{-1}$; ${ }^{1} \mathrm{H}$ NMR $\left(400 \mathrm{MHz}, \mathrm{CDCl}_{3}\right) \delta: 6.52(\mathrm{~s}, 1 \mathrm{H}), 3.84-3.83(\mathrm{~m}, 1 \mathrm{H}), 3.83(\mathrm{~s}, 3 \mathrm{H}), 3.43(\mathrm{~s}, 3 \mathrm{H}), 3.12-3.03$ (m, 1H), 3.03-2.98 (m, 1H), $2.94(\mathrm{dd}, J=9.7,5.5 \mathrm{~Hz}, 1 \mathrm{H}), 2.62(\mathrm{dd}, J=15.6,3.8 \mathrm{~Hz}, 1 \mathrm{H}), 2.53-2.47$ (m, 1H), $2.45(\mathrm{~s}, 3 \mathrm{H}), 2.31-2.25(\mathrm{~m}, 1 \mathrm{H}), 2.01(\mathrm{~d}, J=12.3 \mathrm{~Hz}, 1 \mathrm{H}), 1.86-1.78(\mathrm{~m}, 2 \mathrm{H}), 1.77-1.69(\mathrm{~m}$, 1H), 1.73-1.66 (m, 2H), 1.59-1,53 (m, 1H), 1.48-1.33 (m, 2H); ${ }^{13} \mathrm{C}$ NMR (101 MHz, $\left.\mathrm{CDCl}_{3}\right) \delta: 146.0$, 142.1, 126.2, 124.6, 123.9, 109.8, 102.0, 70.8, 60.0, 55.9, 537, 49.2, 43.7, 34.7, 34.3, 34.2, 33.1, 28.9, 27.1, 26.4; HRMS $(\mathrm{m} / \mathrm{z})$ : calcd for $\mathrm{C}_{20} \mathrm{H}_{28} \mathrm{NO}_{4}\left([\mathrm{M}+\mathrm{H}]^{+}\right) 346.2013$, found 346.2011.

The product $(+)-1(20.0 \mathrm{mg})$ was re-dissolved in a mixed solvent of ethyl acetate $(1 \mathrm{~mL})$ and $n$-hexane $(1 \mathrm{~mL})$. After slowly evaporating the solvents at ambient temperautre, the fine crystals were obtained amd their structure was analyzed by X-ray diffraction.

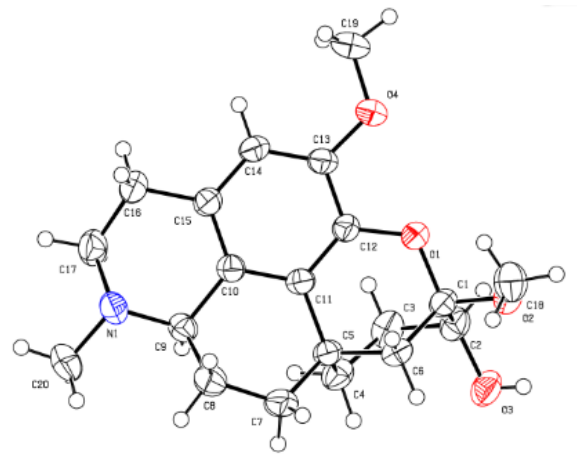


Table S2. Crystal data and structure refinement for (+)-regeline (1) (CCDC 2003662)

\begin{tabular}{|c|c|}
\hline Empirical formula & $\mathrm{C}_{20} \mathrm{H}_{27} \mathrm{NO}_{4}$ \\
\hline Formula weight & 345.42 \\
\hline Temperature/K & 294.15 \\
\hline Crystal system & trigonal \\
\hline Space group & $\mathrm{P} 3_{2}$ \\
\hline $\mathrm{a} / \AA$ & $11.34890(10)$ \\
\hline $\mathrm{b} / \AA$ & $11.34890(10)$ \\
\hline$c / \AA ̊$ & $12.20100(10)$ \\
\hline$\alpha /^{\circ}$ & 90 \\
\hline$\beta /{ }^{\circ}$ & 90 \\
\hline$\gamma /{ }^{\circ}$ & 120 \\
\hline Volume $/ \AA^{3}$ & $1360.92(3)$ \\
\hline $\mathrm{Z}$ & 3 \\
\hline$\rho_{\text {calc }} \mathrm{g} / \mathrm{cm}^{3}$ & 1.264 \\
\hline$\mu / \mathrm{mm}^{-1}$ & 0.706 \\
\hline $\mathrm{F}(000)$ & 558.0 \\
\hline Crystal size $/ \mathrm{mm}^{3}$ & $0.34 \times 0.26 \times 0.24$ \\
\hline Radiation & $\mathrm{CuK} \alpha(\lambda=1.54184)$ \\
\hline $2 \Theta$ range for data collection $/{ }^{\circ}$ & 8.998 to 158.73 \\
\hline Index ranges & $-14 \leq \mathrm{h} \leq 14,-13 \leq \mathrm{k} \leq 13,-15 \leq 1 \leq 15$ \\
\hline Reflections collected & 11417 \\
\hline Independent reflections & $3640\left[R_{\text {int }}=0.0195, R_{\text {sigma }}=0.0157\right]$ \\
\hline Data/restraints/parameters & $3640 / 1 / 234$ \\
\hline Goodness-of-fit on $\mathrm{F}^{2}$ & 1.044 \\
\hline Final $R$ indexes $[I>=2 \sigma(I)]$ & $\mathrm{R}_{1}=0.0302, \mathrm{wR}_{2}=0.0869$ \\
\hline Final $R$ indexes [all data] & $\mathrm{R}_{1}=0.0304, \mathrm{wR}_{2}=0.0871$ \\
\hline Largest diff. peak/hole / e $\AA^{-3}$ & $0.24 /-0.15$ \\
\hline Flack parameter & $0.04(6)$ \\
\hline
\end{tabular}


Table S3. Comparison of NMR data of natural and synthetic (+)-regeline

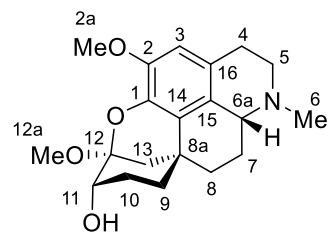

(+)-regeline

\begin{tabular}{|c|c|c|}
\hline \multirow{2}{*}{ No. } & \multicolumn{2}{|c|}{${ }^{1} \mathrm{H}$ NMR $\left(\mathrm{CDCl}_{3}\right) \delta:$} \\
\hline & Natural $^{[2]}$ & Synthetic (400 MHz) \\
\hline 3 & $6.42(\mathrm{~s})$ & $6.52(\mathrm{~s})$ \\
\hline $6 a$ & - & $2.94(\mathrm{dd}, J=9.7,5.5 \mathrm{~Hz})$ \\
\hline 11 & - & $3.84-3.83(\mathrm{~m})$ \\
\hline $\mathrm{O}-\mathrm{Me}$ & $3.74(\mathrm{~s})$ & $3.83(\mathrm{~s})$ \\
\hline $\mathrm{O}-\mathrm{Me}$ & $3.34(\mathrm{~s})$ & $3.43(\mathrm{~s})$ \\
\hline \multirow[t]{3}{*}{$\mathrm{N}-\mathrm{Me}$} & $2.36(\mathrm{~s})$ & $2.45(\mathrm{~s})$ \\
\hline & \multicolumn{2}{|c|}{${ }^{13} \mathrm{C}$ NMR $\left(\mathrm{CDCl}_{3}\right) \delta:$} \\
\hline & Natural $^{[2]}$ & Synthetic (101 MHz) \\
\hline 1 & 145.0 & 146.0 \\
\hline 2 & 142.1 & 142.1 \\
\hline 14 & 126.4 & 126.2 \\
\hline 15 & 124.6 & 124.6 \\
\hline 16 & 123.8 & 123.9 \\
\hline 3 & 110.2 & 109.8 \\
\hline 12 & 101.9 & 102.0 \\
\hline 11 & 70.5 & 70.8 \\
\hline $6 a$ & 60.0 & 60.0 \\
\hline $2 \mathrm{a}$ & 56.0 & 55.9 \\
\hline 5 & 53.6 & 53.7 \\
\hline $12 \mathrm{a}$ & 48.9 & 49.2 \\
\hline 6 & 43.6 & 43.7 \\
\hline $8 \mathrm{a}$ & 34.6 & 34.7 \\
\hline $4,13,10,9,8,7$ & $34.2,34.2,33.0,28.8,27.0,26.4$ & $34.3,34.2,33.1,28.9,27.1,26.4$ \\
\hline
\end{tabular}

\section{Synthesis of (+)-regalamine (2)}

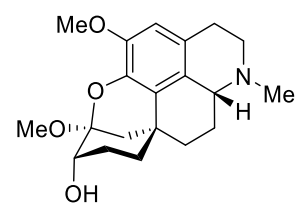

$(+)$-regeline (1)

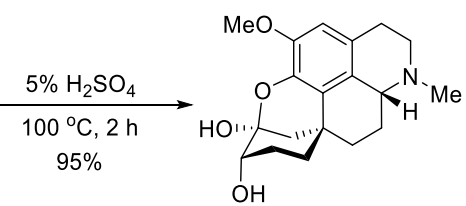

$(+)$-regalamine (2)

The mixture of (+)-1 (44.0 mg, $0.13 \mathrm{mmol})$ and $2 \mathrm{~mL}$ of $5 \% \mathrm{H}_{2} \mathrm{SO}_{4}$ was heated to $100{ }^{\circ} \mathrm{C}$ and stirred at the same temperature for $2 \mathrm{~h}$. The reaction mixture was then cooled to room temperature, and a saturated aqueous solution of $\mathrm{Na}_{2} \mathrm{CO}_{3}(4 \mathrm{~mL})$ was added to quench the reaction. The mixture was extracted with $\mathrm{CH}_{2} \mathrm{Cl}_{2}(3 \times 5 \mathrm{~mL})$. The combined organic phase was dried over anhydrous $\mathrm{Na}_{2} \mathrm{SO}_{4}$ and concentrated in vacuo. The residue was chromatographed on silica-gel column with ethyl acetate/ 
triethylamine (20:1) to give the product (+)-regalamine (2) $(41.0 \mathrm{mg}, 95 \%$ yield) as a white solid: $\mathrm{mp}$ $220-222{ }^{\circ} \mathrm{C}$ (lit. $\left.{ }^{3} 225-226^{\circ} \mathrm{C}\right) ; R_{\mathrm{f}} 0.15$ (ethyl acetate/triethylamine $\left.=30: 1\right) ;[\alpha]_{\mathrm{D}}^{25}+43.8(c 1.92, \mathrm{MeOH})$ (lit. $^{3}+33$ (c 1.93, MeOH)); IR (KBr): $v_{\max }=3464,3370,2938,2858,2794,1599,1485,1446,1373$, $1265,1224,1174,1138,1055,1013,875,734,701 \mathrm{~cm}^{-1} ;{ }^{1} \mathrm{H}$ NMR $\left(400 \mathrm{MHz}, \mathrm{CDCl}_{3}\right) \delta: 6.45(\mathrm{~s}, 1 \mathrm{H})$, 3.83-3.82 (m, 1H), $3.81(\mathrm{~s}, 3 \mathrm{H}), 3.13-3.09(\mathrm{~m}, 1 \mathrm{H}), 3.00-2.98(\mathrm{~m}, 1 \mathrm{H}), 2.94(\mathrm{dd}, J=10.0,5.6 \mathrm{~Hz}, 1$ H), 2.59 (dd, $J=16.4,3.2 \mathrm{~Hz}, 1 \mathrm{H}), 2.49(\mathrm{td}, J=11.6,3.6 \mathrm{~Hz}, 1 \mathrm{H}), 2.44(\mathrm{~s}, 3 \mathrm{H}), 2.29-2.26(\mathrm{~m}, 1 \mathrm{H})$, $2.20(\mathrm{~d}, J=12.4 \mathrm{~Hz}, 1 \mathrm{H}), 1.72-1.69(\mathrm{~m}, 4 \mathrm{H}), 1.52-1.48$ (m, $1 \mathrm{H}), 1.48-1.45(\mathrm{~m}, 1 \mathrm{H}), 1.38-1.35(\mathrm{~m}$, $1 \mathrm{H}) ;{ }^{13} \mathrm{C}$ NMR (101 MHz, $\left.\mathrm{CDCl}_{3}\right) \delta: 145.6,141.4,126.2,124.7,123.6,109.5,99.4,71.4,60.1,55.8$, 53.6, 43.6, 39.0, 34.9, 33.9, 32.8, 28.7, 26.9, 26.5; HRMS $(m / z)$ : calcd for $\mathrm{C}_{19} \mathrm{H}_{26} \mathrm{NO}_{4}\left([\mathrm{M}+\mathrm{H}]^{+}\right)$ 332.1856 , found 332.1863 .

\section{Synthesis of (+)-kesselridine (3)}

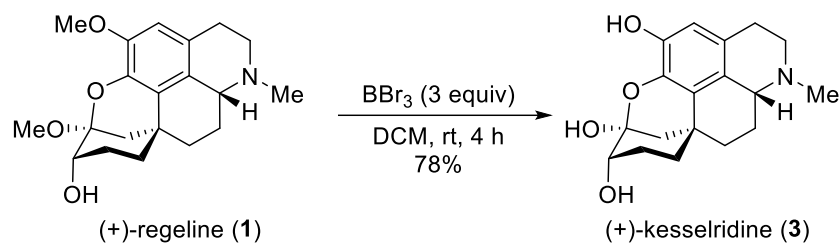

To a solution of (+)-regeline (20.0 mg, $57.9 \mu \mathrm{mol})$ in $\mathrm{CH}_{2} \mathrm{Cl}_{2}(3 \mathrm{~mL})$ was added $\mathrm{BBr}_{3}(0.17 \mathrm{~mL}$, $1.0 \mathrm{M}, 0.17 \mathrm{mmol}$ ) in $\mathrm{CH}_{2} \mathrm{Cl}_{2}$, and the resulting mixture was stirred at room temperature for $4 \mathrm{~h}$. The reaction mixture was diluted with saturated aqueous solution of $\mathrm{NaHCO}_{3}(5 \mathrm{~mL})$ and extracted with $\mathrm{CH}_{2} \mathrm{Cl}_{2}(3 \times 8 \mathrm{~mL})$. The combined organic phase was dried over anhydrous $\mathrm{Na}_{2} \mathrm{SO}_{4}$ and concentrated in vacuo. The residue was chromatographed on silica-gel column with ethyl acetate/triethylamine (20:1) to give the product (+)-kesselridine (3) $\left(14.0 \mathrm{mg}, 78 \%\right.$ yield) as a white solid: $\mathrm{mp} 205-206{ }^{\circ} \mathrm{C}$ (lit. ${ }^{4}$ $232-234{ }^{\circ} \mathrm{C}$ ); $R_{\mathrm{f}} 0.22$ (ethyl acetate/triethylamine $=30: 1$ ); $[\alpha]_{\mathrm{D}}^{25}+200.0\left(c 0.1\right.$, pyridine) (lit. ${ }^{4}-50$ (pyridine)); IR (KBr): $v_{\max }=3737,3651,3626,2955,2918,2850,1461,1377,1237,1131,992,966$, 895, $853 \mathrm{~cm}^{-1}$; ${ }^{1} \mathrm{H}$ NMR $\left(400 \mathrm{MHz}, \mathrm{CD}_{3} \mathrm{OD}\right) \delta: 6.45(\mathrm{~s}, 1 \mathrm{H}), 3.71-3.70(\mathrm{~m}, 1 \mathrm{H}), 3.07-3.01(\mathrm{~m}, 2 \mathrm{H})$, 3.01-2.92 (m, 1H), 2.63-2.54 (m, 2H), $2.46(\mathrm{~s}, 3 \mathrm{H}), 2.34-2.26(\mathrm{~m}, 1 \mathrm{H}), 2.16(\mathrm{~d}, J=12.8 \mathrm{~Hz}, 1 \mathrm{H})$, $1.80-1.68(\mathrm{~m}, 3 \mathrm{H}), 1.65-1.58(\mathrm{~m}, 1 \mathrm{H}), 1.56(\mathrm{~d}, J=12.4 \mathrm{~Hz}, 1 \mathrm{H}), 1.46-1.42(\mathrm{~m}, 1 \mathrm{H}), 1.40-1.33(\mathrm{~m}$, 1H), 1.32-1.27 (m, 1H); ${ }^{13} \mathrm{C}$ NMR (101 MHz, $\left.\mathrm{CD}_{3} \mathrm{OD}\right) \delta: 144.5,142.4,125.0,124.8,123.9,114.2,100$ 8, 72.1, 61.7, 54.5, 42.6, 39.6, 35.8, 34.8, 34.3, 28.2(2), 27.1; HRMS ( $/ \mathrm{z} / \mathrm{z})$ : calcd for $\mathrm{C}_{19} \mathrm{H}_{26} \mathrm{NO}_{4}([\mathrm{M}+$ $\left.\mathrm{H}]^{+}\right) 318.1700$, found 318.1699 .

\section{Synthesis of $(+)$-kesselringine (4)}

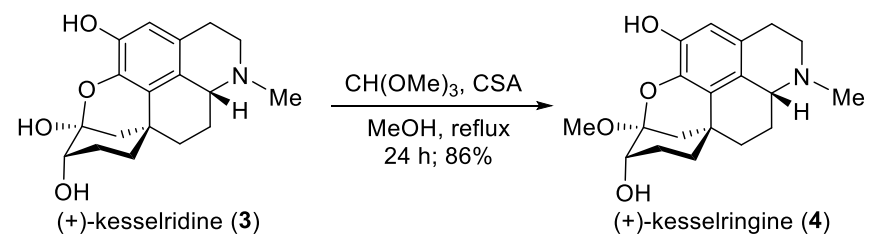

To a solution of $(+)-3(28.0 \mathrm{mg}, 88.2 \mu \mathrm{mol})$ in $\mathrm{MeOH}(5 \mathrm{~mL})$ were added $\mathrm{CH}(\mathrm{OMe})_{3}(30.0 \mu \mathrm{L}$, $0.27 \mathrm{mmol}$ ) and (+)-10-camphorsulfonic acid (CSA, $30.0 \mathrm{mg}, 0.13 \mathrm{mmol})$. The reaction mixture was heated to reflux for $24 \mathrm{~h}$, diluted with saturated aqueous $\mathrm{NaHCO}_{3}$ solution $(10 \mathrm{~mL})$, and extracted with EtOAc $(3 \times 6 \mathrm{~mL})$. The combined organic phase was dried over anhydrous $\mathrm{Na}_{2} \mathrm{SO}_{4}$ and concentrated in vacuo. The residue was chromatographed on silica-gel column with ethyl acetate/triethylamine (20:1) to give the product (+)-kesselringine (4) $\left(25.0 \mathrm{mg}, 86 \%\right.$ yield) as a white solid: $\mathrm{mp} 197-198{ }^{\circ} \mathrm{C}$ (lit. ${ }^{5}$ 
$\left.194-196{ }^{\circ} \mathrm{C}\right) ; R_{\mathrm{f}} 0.25$ (ethyl acetate/triethylamine $\left.=30: 1\right) ;[\alpha]_{\mathrm{D}}^{25}+79.0\left(c 1.0, \mathrm{CHCl}_{3}\right)\left(\mathrm{lit}^{6}{ }^{6}+93(c 1.0\right.$, $\left.\mathrm{CHCl}_{3}\right)$ ); IR (KBr): $v_{\max }=3473,2953,2928,2868,1595,1503,1462,1377,1272,1189,1059,813$, 723, $698 \mathrm{~cm}^{-1} ;{ }^{1} \mathrm{H}$ NMR (400 MHz, $\left.\mathrm{CDCl}_{3}\right) \delta: 6.56(\mathrm{~s}, 1 \mathrm{H}), 5.51$ (brs, $\left.1 \mathrm{H}\right), 3.75(\mathrm{~m}, 1 \mathrm{H}), 3.42(\mathrm{~s}, 3 \mathrm{H})$, 3.13-2.89 (m, 3H), 2.65-2.50 (m, 2H), $2.46(\mathrm{~s}, 3 \mathrm{H}), 2.36-2.23(\mathrm{~m}, 2 \mathrm{H}), 2.02(\mathrm{~d}, J=12.4 \mathrm{~Hz}, 1 \mathrm{H})$, $1.88-1.68(\mathrm{~m}, 5 \mathrm{H}), 1.57-1.49(\mathrm{~m}, 1 \mathrm{H}), 1.46-1.31(\mathrm{~m}, 2 \mathrm{H}) ;{ }^{13} \mathrm{C} \mathrm{NMR}\left(101 \mathrm{MHz}, \mathrm{CDCl}_{3}\right) \delta: 142.2$, 139.9, 125.4, 123.2, 112.5, 102.8, 77.2, 70.9, 60.2, 53.6, 49.4, 43.5, 34.6, 34.1, 34.0, 33.3, 28.4, 27.1, 26.3; HRMS $(m / z)$ : calcd for $\mathrm{C}_{19} \mathrm{H}_{26} \mathrm{NO}_{4}\left([\mathrm{M}+\mathrm{H}]^{+}\right) 332.1856$, found 332.1859 .

\section{Synthesis of compound (+)-jolantidine (5)}

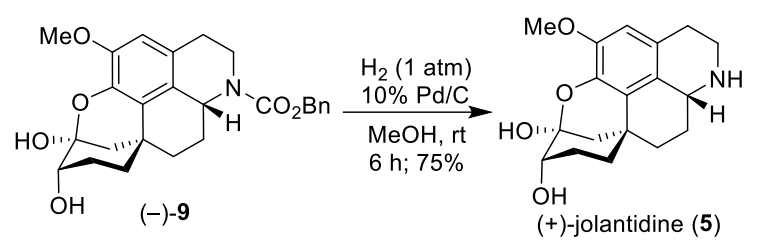

To a solution of compound (-)-9 (41.0 mg, $91.8 \mu \mathrm{mol})$ in $\mathrm{MeOH}(5 \mathrm{~mL})$ was added $\mathrm{Pd} / \mathrm{C}(9.0 \mathrm{mg})$, and the suspension mixture was stirred at room temperature under ambient $\mathrm{H}_{2}$ pressure for $6 \mathrm{~h}$. The mixture was then filtered through a pad of Florisil and concentrated in vacuo. The residue was chromatographed on silica-gel column with dichloromethane/methanol $(3: 1)$ to give the product (+)-jolantidine (5) (21.0 mg, 75\% yield) as a white solid: $\mathrm{mp} 269-270{ }^{\circ} \mathrm{C}\left(\right.$ lit. $\left.{ }^{7} 275-277{ }^{\circ} \mathrm{C}\right) ; R_{\mathrm{f}} 0.53$ (dichloromethane/methanol = 3:1); $[\alpha]_{\mathrm{D}}^{25}+90.5\left(c\right.$ 0.4, MeOH) $\left(\right.$ lit. ${ }^{7}+102$ (c 0.4, MeOH)); IR $(\mathrm{KBr})$ : $v_{\max }=3454,3298,2925,2853,1598,1484,1445,1368,1265,1173,1051,1013,737,702 \mathrm{~cm}^{-1} ;{ }^{1} \mathrm{H}$ NMR $\left(400 \mathrm{MHz}, \mathrm{CD}_{3} \mathrm{OD}\right) \delta: 6.61(\mathrm{~s}, 1 \mathrm{H}), 3.77(\mathrm{~s}, 3 \mathrm{H}), 3.75-3.72(\mathrm{~m}, 1 \mathrm{H}), 3.72-3.70(\mathrm{~m}, 1 \mathrm{H})$, 3.26-3.19 (m, 1H), $3.02(\mathrm{td}, J=11.2,4.4 \mathrm{~Hz}, 1 \mathrm{H}), 2.98-2.88(\mathrm{~m}, 1 \mathrm{H}), 2.71-2.65(\mathrm{~m}, 1 \mathrm{H}), 2.18(\mathrm{~d}, J=$ $12.4 \mathrm{~Hz}, 1 \mathrm{H}), 2.15-2.09(\mathrm{~m}, 1 \mathrm{H}), 1.83-1.67(\mathrm{~m}, 3 \mathrm{H}), 1.65-1.58(\mathrm{~m}, 1 \mathrm{H}), 1.54(\mathrm{~d}, J=12.4 \mathrm{~Hz}, 1 \mathrm{H})$, $1.47-1.35$ (m, 3H); ${ }^{13} \mathrm{C}$ NMR (101 MHz, $\left.\mathrm{CD}_{3} \mathrm{OD}\right) \delta: 147.4,143.6,127.6,125.8,124.8,111.9,100.7$, 72.1, 56.5, 52.5, 44.0, 39.5, 36.0, 34.8, 34.5, 29.2, 28.8, 28.2; HRMS $(\mathrm{m} / \mathrm{z})$ : calcd for $\mathrm{C}_{18} \mathrm{H}_{24} \mathrm{NO}_{4}([\mathrm{M}+$ $\left.\mathrm{H}]^{+}\right) 318.1700$, found 318.1697 .

\section{Synthesis of $(+)$-regelinine $(6)$}

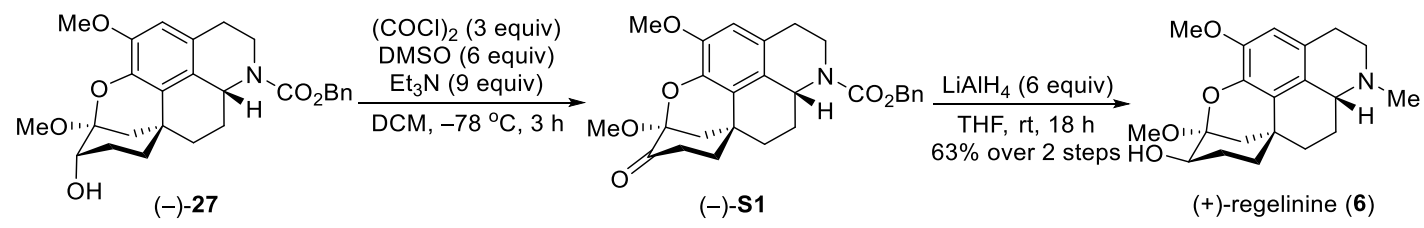

To a solution of oxalyl chloride $(0.10 \mathrm{~mL}, 1.10 \mathrm{mmol})$ in $\mathrm{CH}_{2} \mathrm{Cl}_{2}(1 \mathrm{~mL})$ was added slowly the solution of DMSO $(0.16 \mathrm{~mL}, 2.20 \mathrm{mmol})$ in $\mathrm{CH}_{2} \mathrm{Cl}_{2}(1 \mathrm{~mL})$ at $-78{ }^{\circ} \mathrm{C}$. A solution of $(-)-27(0.17 \mathrm{~g}$, $0.37 \mathrm{mmol})$ in $\mathrm{CH}_{2} \mathrm{Cl}_{2}(3 \mathrm{~mL})$ was added dropwise at the same temperature and the reaction mixture was stirred for $2 \mathrm{~h}$. Subsequently, triethylamine $(0.46 \mathrm{~mL}, 3.30 \mathrm{mmol})$ was added dropwise at $-78{ }^{\circ} \mathrm{C}$. The reaction mixture was stirred for $1 \mathrm{~h}$, warmed to room temperature naturally, and quenched with water $(5 \mathrm{~mL})$, extracted with $\mathrm{CH}_{2} \mathrm{Cl}_{2}(3 \times 10 \mathrm{~mL})$. The combined organic phase was dried over $\mathrm{Na}_{2} \mathrm{SO}_{4}$, and concentrated in vacuo. The residue was chromatographed on silica-gel column with petroleum ether/ethyl acetate (2:1) to give the product (-)-S1 $\left(0.16 \mathrm{~g}, 96 \%\right.$ yield) as a white solid, mp $75-77{ }^{\circ} \mathrm{C}$; $R_{\mathrm{f}} 0.43$ (petroleum ether/ethyl acetate $\left.=1: 1\right) ;[\alpha]_{\mathrm{D}}^{25}+150\left(c 1.0, \mathrm{CHCl}_{3}\right) ; \mathrm{IR}(\mathrm{KBr}): v_{\max }=2945,2840$, $1734,1697,1488,1424,1278,1206,1085,983,735,699$ cm-1; 'H NMR (400 MHz, $\left.\mathrm{CDCl}_{3}\right) \delta: 7.44$ 
$7.30(\mathrm{~m}, 5 \mathrm{H}), 6.67(\mathrm{dd}, J=10.0,3.2 \mathrm{~Hz}, 0.2), 6.66 \square 6.57(\mathrm{~m}, 1 \mathrm{H}), 5.88(\mathrm{~m}, 0.2 \mathrm{H}), 5.30-5.09(\mathrm{~m}, 2 \mathrm{H})$, $4.95(\mathrm{~m}, 0.2 \mathrm{H}), 4.82(\mathrm{~m}, 0.6 \mathrm{H}), 4.57(\mathrm{~m}, 0.13 \mathrm{H}), 4.43(\mathrm{~d}, J=12.4 \mathrm{~Hz}, 0.69 \mathrm{H}), 3.85(\mathrm{~m}, 3 \mathrm{H}), 3.62(\mathrm{~m}$, $3 \mathrm{H}), 3.49 \square 3.28(\mathrm{~m}, 0.65 \mathrm{H}), 2.97-2.74(\mathrm{~m}, 2 \mathrm{H}), 2.66-2.56(\mathrm{~m}, 0.8 \mathrm{H}), 2.46-2.12(\mathrm{~m}, 5.1 \mathrm{H}), 2.00-1.72$ (m, 3.5H), 1.63-1.46 (m, 0.9H); ${ }^{13} \mathrm{C}$ NMR (101 MHz, $\left.\mathrm{CDCl}_{3}\right) \delta: 202.1,156.4,146.6,141.1,136.8$, 128.6, 128.1(2), 123.8, 121.6, 112.6, 111.1, 99.3, 67.2, 56.2, 51.2, 42.7, 40.0, 39.1, 37.1, 36.3, 34.9, 34.5, 32.6, 29.9; $\operatorname{HRMS}(\mathrm{m} / \mathrm{z})$ : calcd for $\mathrm{C}_{2} 7 \mathrm{H}_{30} \mathrm{NO}_{6}([\mathrm{M}+\mathrm{H}]+)$ 464.2068, found 464.2066.

To the solution of (-)-S1 in THF $(8 \mathrm{~mL})$ was added $\mathrm{LiAlH}_{4}(83.5 \mathrm{mg}, 2.20 \mathrm{mmol})$, and the resulting mixture was stirred at room temperature for $24 \mathrm{~h}$. The reaction was then quenched by slow addition of $\mathrm{H}_{2} \mathrm{O}(8 \mathrm{~mL})$, and the mixture was extracted with EtOAc $(3 \times 8 \mathrm{~mL})$. The combined organic phase was dried over anhydrous $\mathrm{Na}_{2} \mathrm{SO}_{4}$ and concentrated in vacuo. The residue was chromatographed on silica-gel column with ethyl acetate/triethylamine (30:1) to give (+)-regelinine (6) $(80.0 \mathrm{mg}, 63 \%$ yield over 2 steps) as a white solid: $\mathrm{mp} \quad 252-253{ }^{\circ} \mathrm{C}$ (lit. ${ }^{8} 253-254{ }^{\circ} \mathrm{C}$ ); $R_{\mathrm{f}} 0.32$ (ethyl acetate/triethylamine $=20: 1) ;[\alpha]_{\mathrm{D}}^{25}+110.4\left(c 1.0, \mathrm{CHCl}_{3}\right) ; \mathrm{IR}(\mathrm{KBr}): v_{\max }=3348,2942,2844,1599$, 1486, 1454, 1244, 1076, 976, 889, $730 \mathrm{~cm}^{-1} ;{ }^{1} \mathrm{H}$ NMR (400 MHz, $\left.\mathrm{CDCl}_{3}\right) \delta: 6.51(\mathrm{~s}, 1 \mathrm{H}), 3.83(\mathrm{~s}, 3 \mathrm{H})$, $3.72(\mathrm{dd}, J=11.2,5.2 \mathrm{~Hz}, 1 \mathrm{H}), 3.41(\mathrm{~s}, 3 \mathrm{H}), 3.12-2.96(\mathrm{~m}, 2 \mathrm{H}), 2.90(\mathrm{dd}, J=10.0,5.2 \mathrm{~Hz}, 1 \mathrm{H}), 2.73-$ $2.56(\mathrm{~m}, 2 \mathrm{H}), 2.50(\mathrm{td}, J=11.2,4.0 \mathrm{~Hz}, 1 \mathrm{H}), 2.44(\mathrm{~s}, 3 \mathrm{H}), 2.30-2.23(\mathrm{~m}, 1 \mathrm{H}), 2.15(\mathrm{dd}, J=12.8,2.8$ $\mathrm{Hz}, 1 \mathrm{H}), 1.92-1.83(\mathrm{~m}, 1 \mathrm{H}), 1.82-1.68(\mathrm{~m}, 3 \mathrm{H}), 1.59-1.47$ (m, 2H), 1.44-1.27 (m, 2H); ${ }^{13} \mathrm{C}$ NMR $(101$ $\left.\mathrm{MHz}, \mathrm{CDCl}_{3}\right) \delta: 146.0,142.4,126.0,124.6,123.5,110.0,100.8,75.5,60.2,55.9,53.7,49.4,43.7,37.6$, 37.4, 34.9, 34.0, 28.9, 28.1, 27.2; HRMS $(\mathrm{m} / \mathrm{z})$ : calcd for $\mathrm{C}_{20} \mathrm{H}_{28} \mathrm{NO}_{4}\left([\mathrm{M}+\mathrm{H}]^{+}\right) 346.2013$, found 346.2013.

\section{References}

1. Chen, J.-Q.; Xie, J.-H.; Bao, D.-H.; Liu, S.; Zhou, Q.-L. Total synthesis of (-)-galanthamine and $(-)$-lycoramine via catalytic asymmetric hydrogenation and intramolecular reductive Heck cyclization. Org. Lett. 2012, 14, 2714-2717.

2. Abdullaeva, D. A.; Yusupov M. K.; Aslanov, K. A. The structure of regeline. Chem. Nat. Comp. 1976, 12, 702-705.

3. Yusupov, M. K.; Abdullaeva, D. A.; Aslanov, K. A.; Sadykov, A. S. The structure of regalamine. Chem. Nat. Comp. 1975, 11, 395-397.

4. Kasimov, K.; Yusupov, M. K.; Timbekov É. K.; Aslanov, K. A. Structure of kesselridine. Chem. Nat. Comp. 1975, 11, 202-205.

5. Yusupov, M. K.; Sadykov, A. S. The structure of kesselringine. Chem. Nat. Comp. 1976, 12, 305-308.

6. Yusupov, M. K.; Mukhamedyarova, N. L.; Sadykov, A. S.; Dolejš, L.; Sedmera P.; Šantavý, F. Constitution of the alkaloid kesselringine. Collect. Czech. Chem. Commun. 1977, 42, 1581-1587.

7. Chommadov, B.; Usmanov, A. M.; Yusupov, M. K. Jolantidine - A new base from Merendera jolantae. Chem. Nat. Comp. 1983, 19, 755.

8. Yusupov, M. K.; Chommadov B.; Aslanov, K. A. The structure of regelinine. Chem. Nat. Comp. 1985, 21, 396-397. 
E) NMR Spectra of New Compounds and Pentacyclic Homoproaporphine Alkaloids

\section{Compound (-)-13}
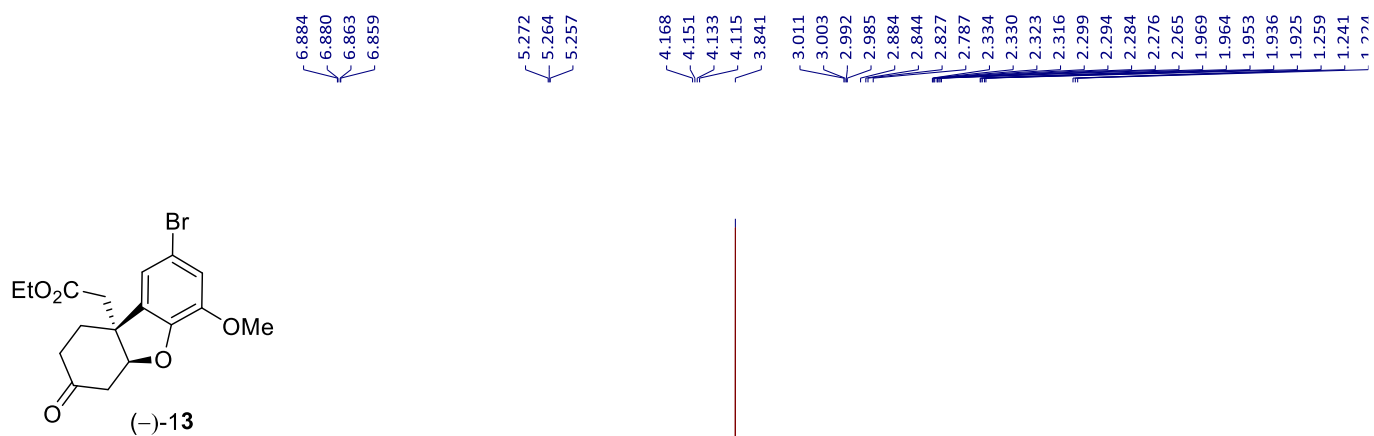

${ }^{1} \mathrm{H} \mathrm{NMR}\left(400 \mathrm{MHz}, \mathrm{CDCl}_{3}\right)$

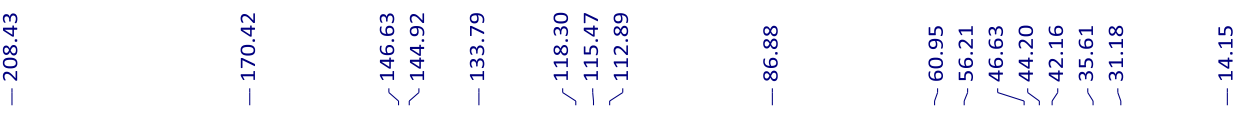

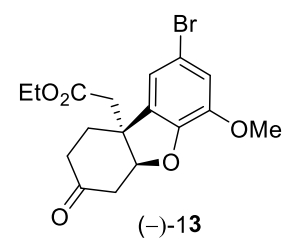

${ }^{13} \mathrm{C}$ NMR $\left(101 \mathrm{MHz}, \mathrm{CDCl}_{3}\right)$

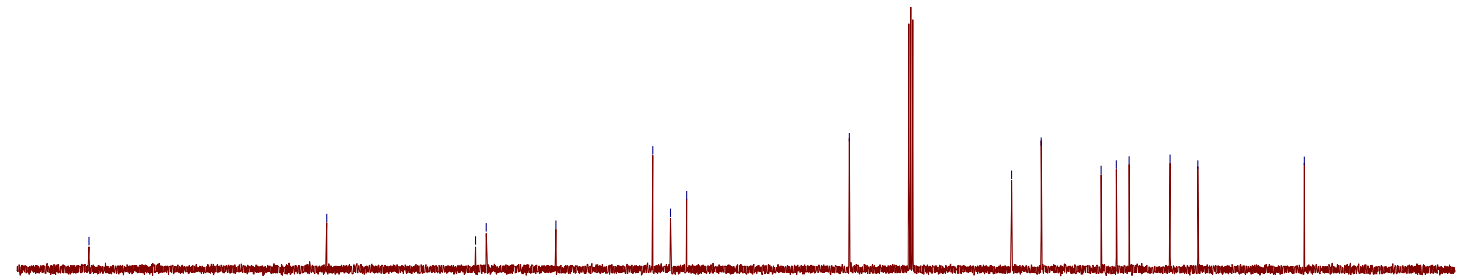

$\begin{array}{lllllllllllllllllllllll}20 & 210 & 200 & 190 & 180 & 170 & 160 & 150 & 140 & 130 & 120 & 110 & 100 & 90 & 80 & 70 & 60 & 50 & 40 & 30 & 20 & 10 & 0\end{array}$ 


\section{Compound 14}

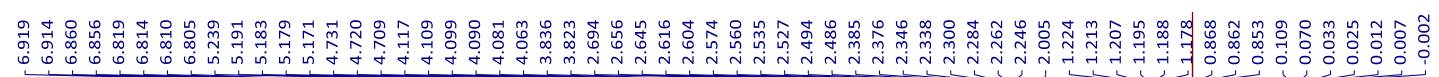

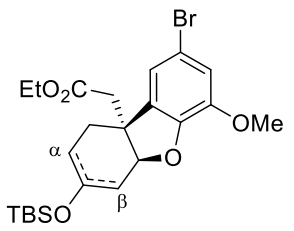

$14 \mathrm{a}(\alpha) / 14 \mathrm{~b}(\beta)$

regioselectivity $5: 1$

${ }^{1} \mathrm{H} \mathrm{NMR}\left(400 \mathrm{MHz}, \mathrm{CDCl}_{3}\right)$
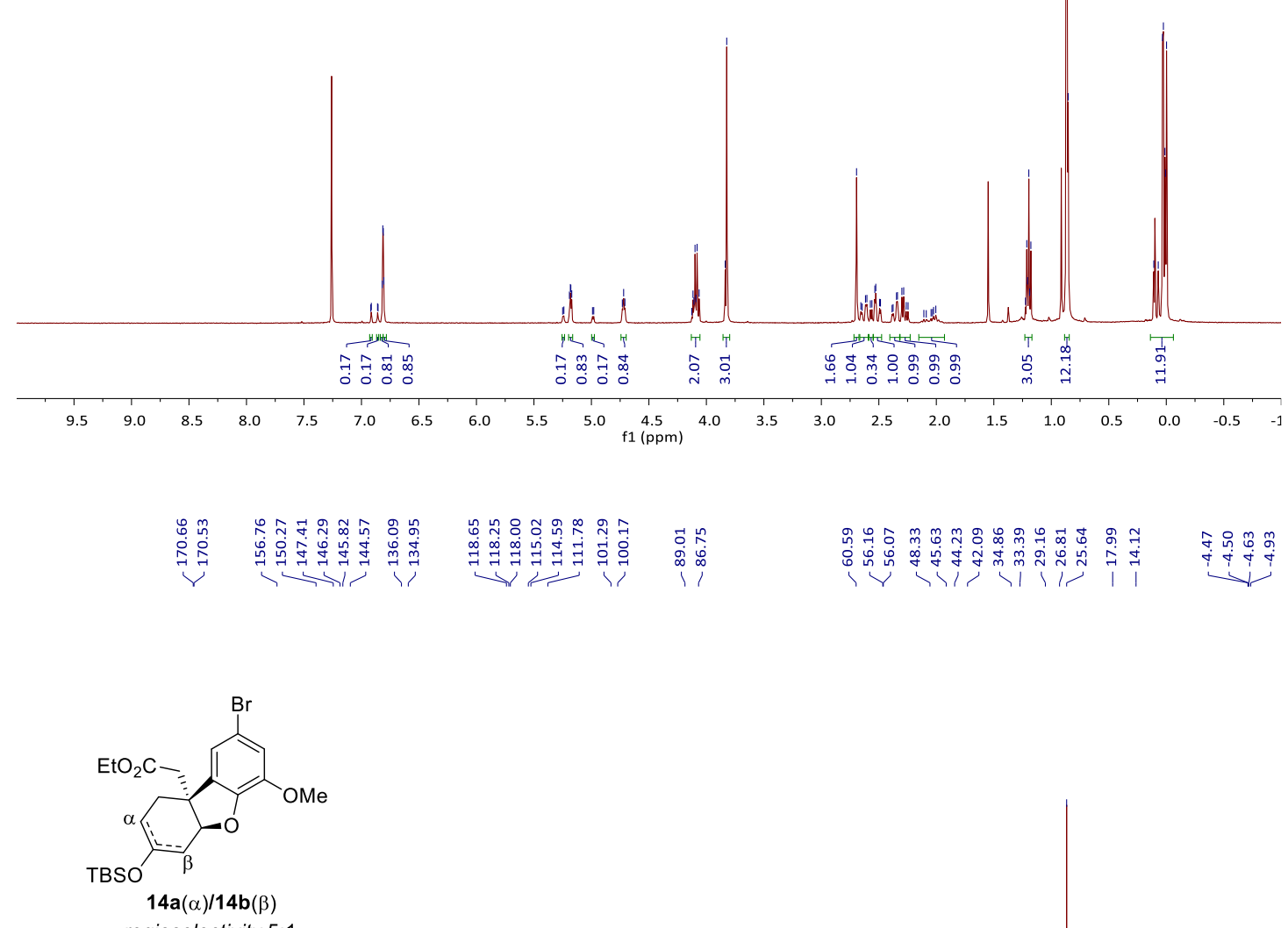

regioselectivity $5: 1$

${ }^{13} \mathrm{C} \mathrm{NMR}\left(101 \mathrm{MHz}, \mathrm{CDCl}_{3}\right)$

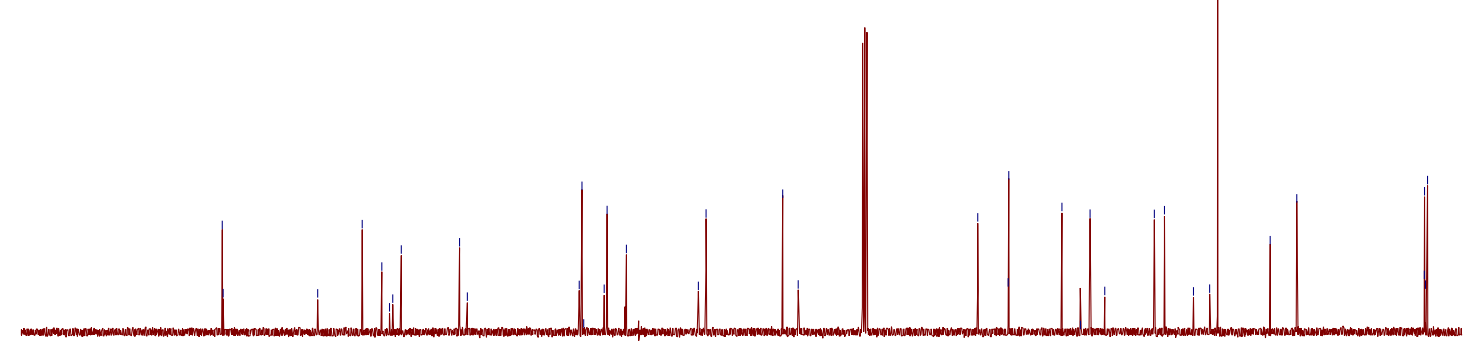

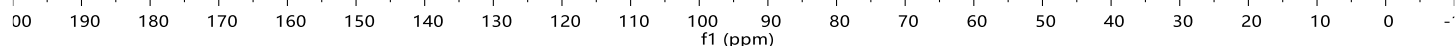


Compound 12 (not stable)

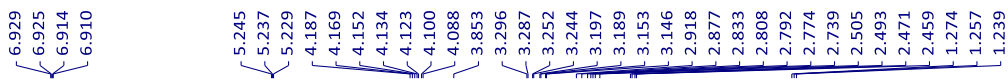

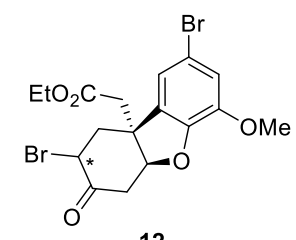

${ }^{1} \mathrm{H} \mathrm{NMR}\left(400 \mathrm{MHz}, \mathrm{CDCl}_{3}\right)$

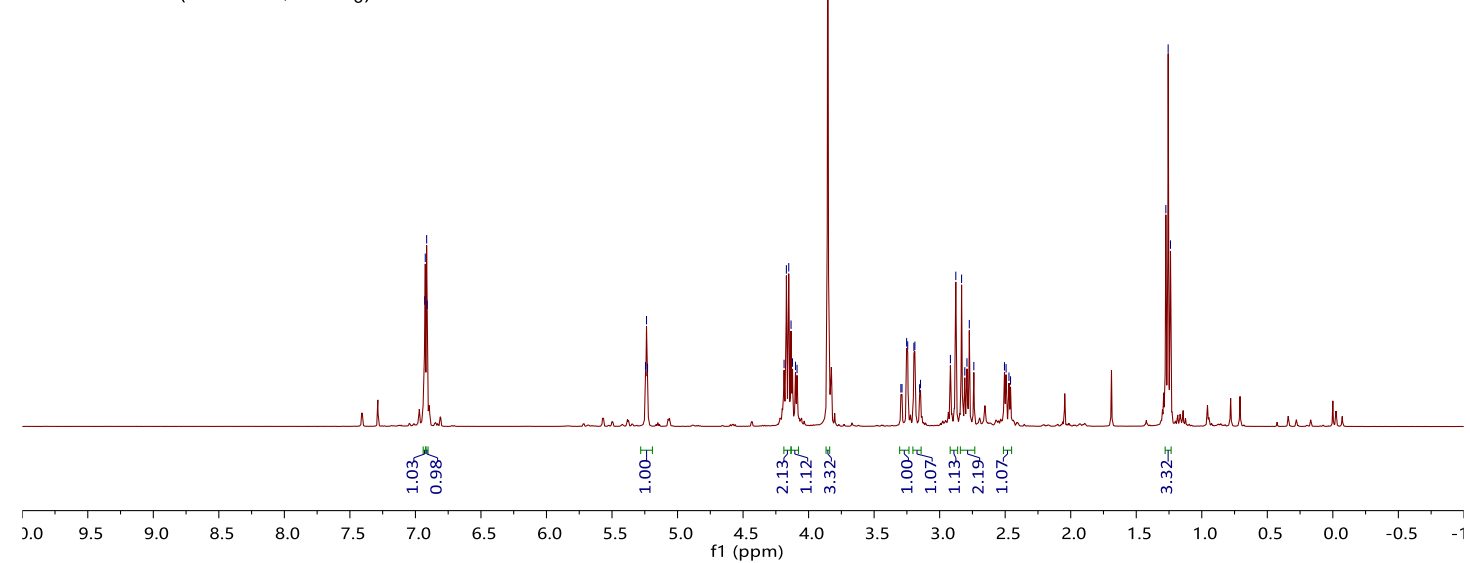

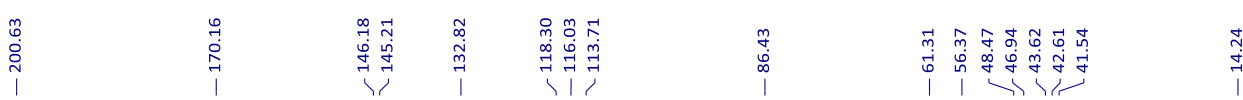

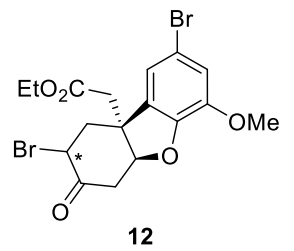

${ }^{13} \mathrm{C}$ NMR $\left(101 \mathrm{MHz}, \mathrm{CDCl}_{3}\right)$

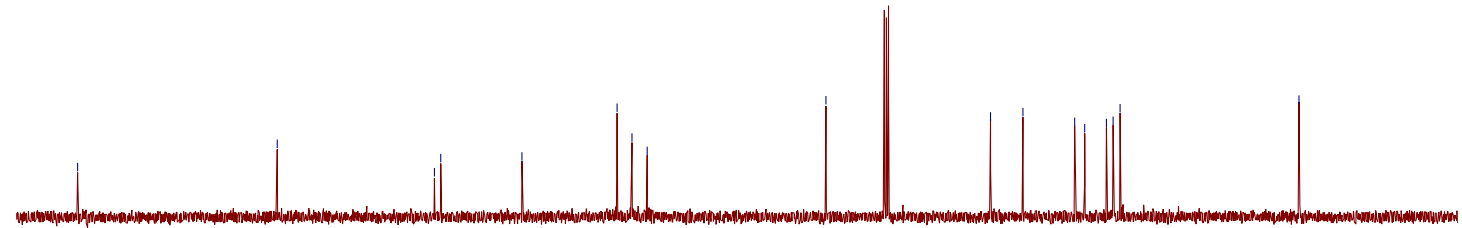

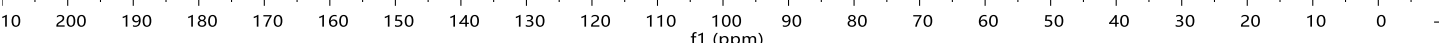




\section{Compound 15a}

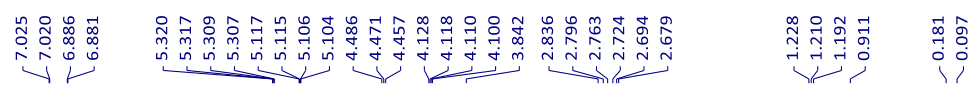

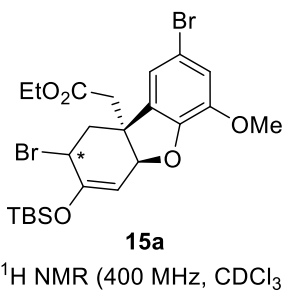

${ }^{1} \mathrm{H}$ NMR (400 MHz, $\mathrm{CDCl}_{3}$ )

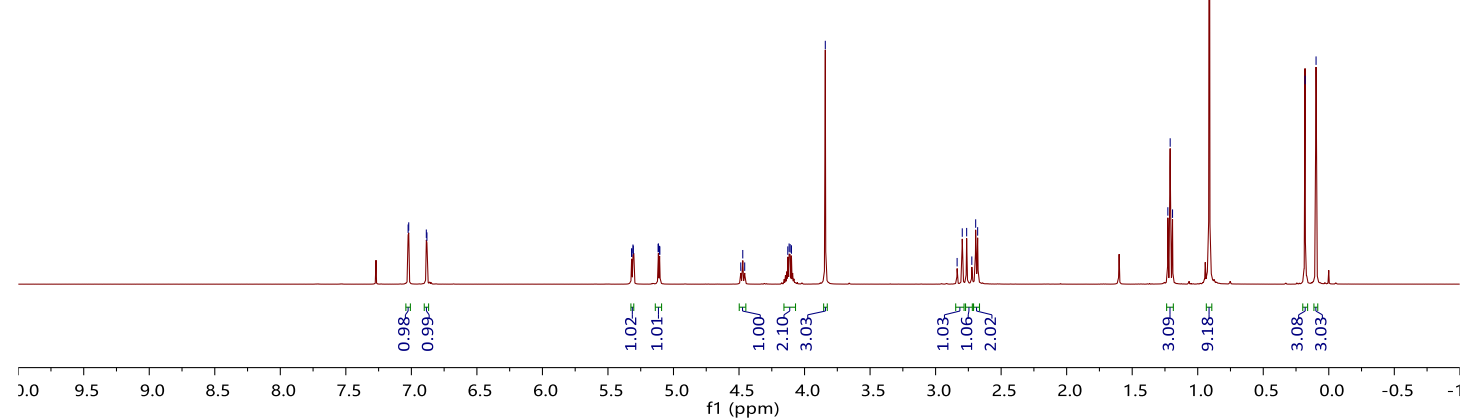

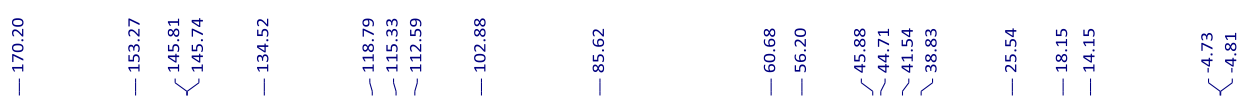

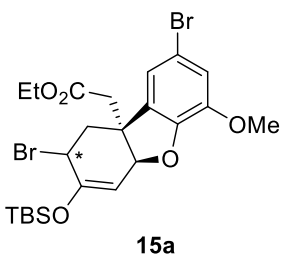

${ }^{13} \mathrm{C}$ NMR $\left(101 \mathrm{MHz}, \mathrm{CDCl}_{3}\right)$

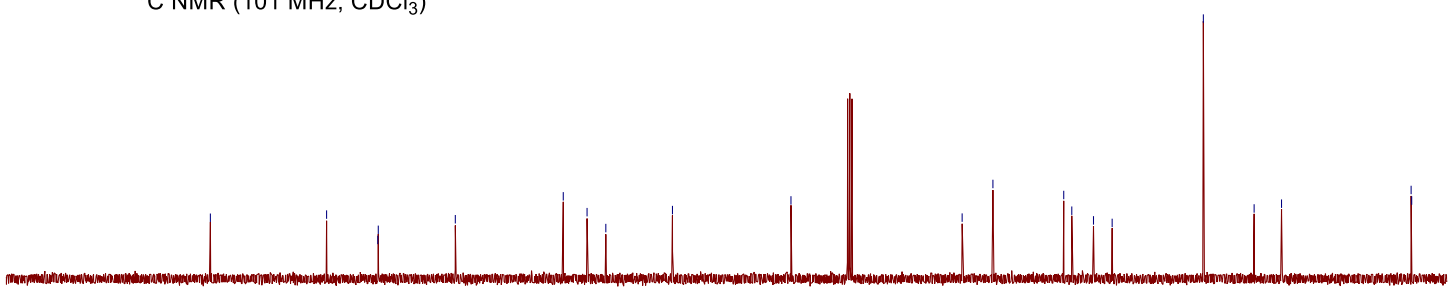

$\begin{array}{lllllllllll}00 & 190 & 180 & 170 & 160 & 150 & 140 & 130 & 120 & 110 & \begin{array}{c}100 \\ \mathrm{f} 1(\mathrm{ppm})\end{array}\end{array}$ 


\section{Compound 15b}

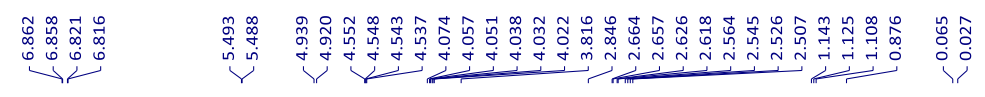

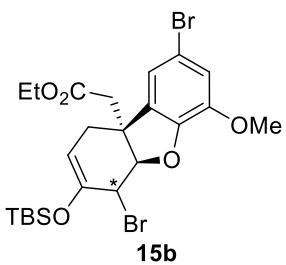

${ }^{1} \mathrm{H}$ NMR $\left(400 \mathrm{MHz}, \mathrm{CDCl}_{3}\right)$

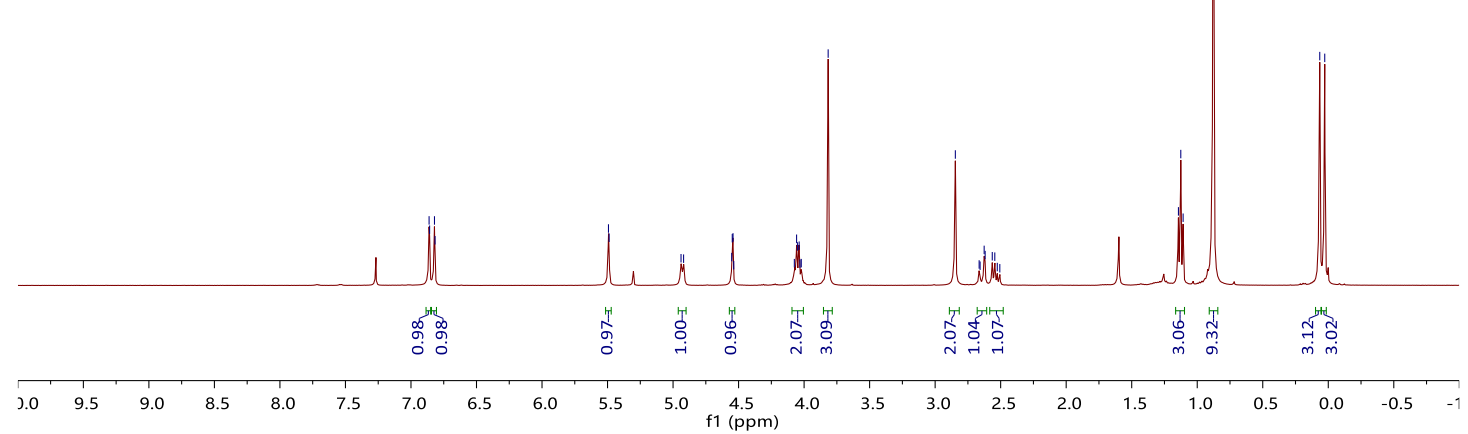

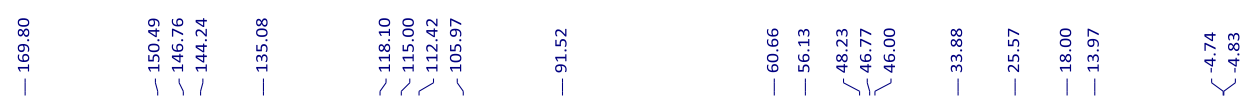

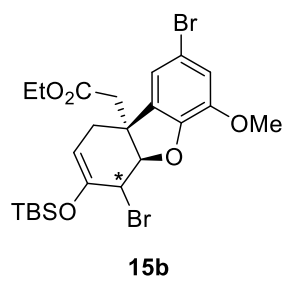

${ }^{13} \mathrm{C}$ NMR $\left(101 \mathrm{MHz}, \mathrm{CDCl}_{3}\right)$ 


\section{Compound (+)-11}

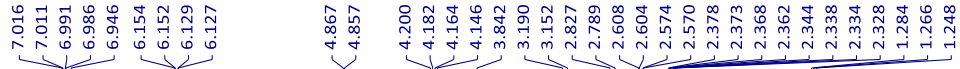

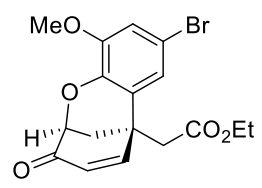

$(+)-11$

${ }^{1} \mathrm{H}$ NMR $\left(400 \mathrm{MHz}, \mathrm{CDCl}_{3}\right)$

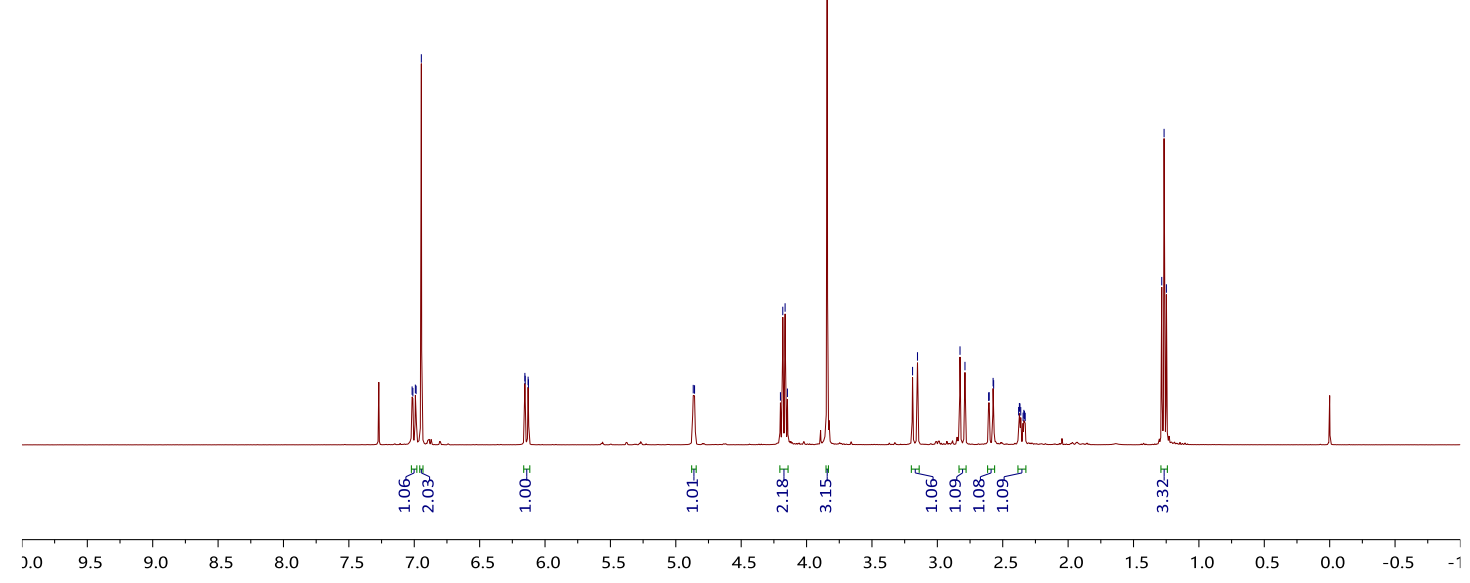

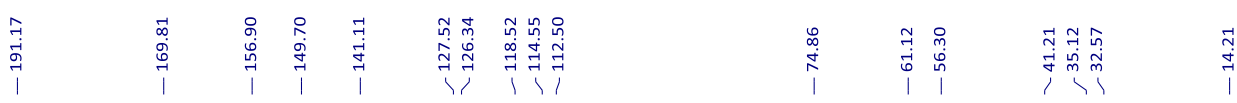

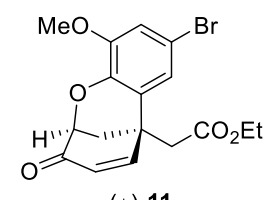

${ }^{13} \mathrm{C}$ NMR $\left(101 \mathrm{MHz}, \mathrm{CDCl}_{3}\right)$

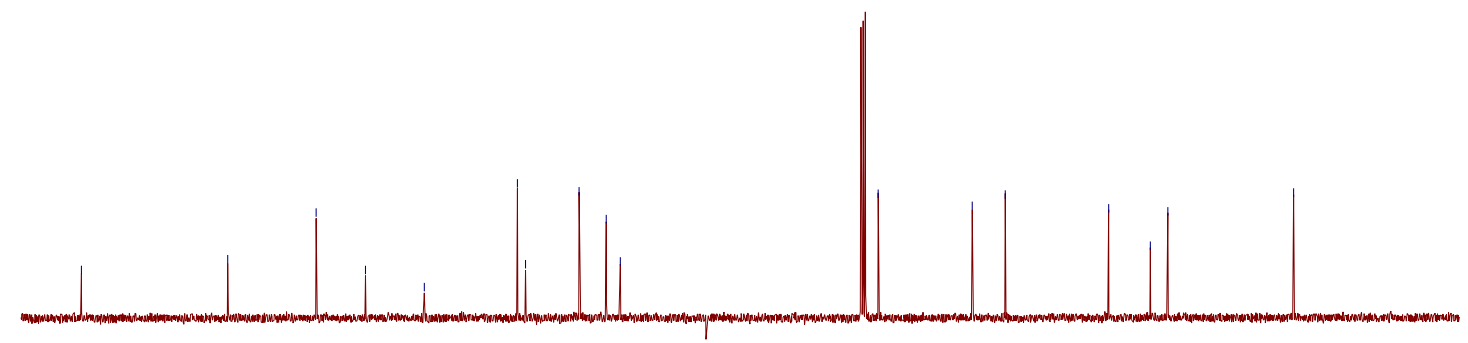

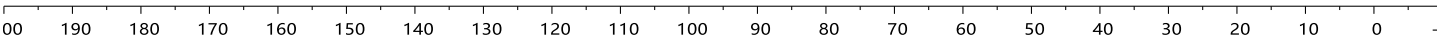




\section{Compound (+)-17}

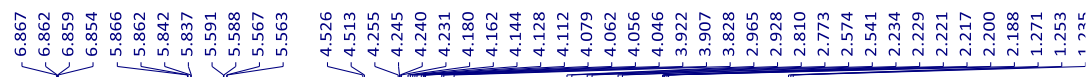

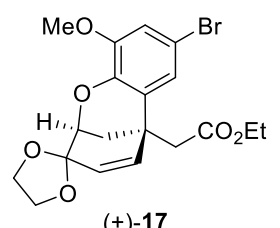

${ }^{1} \mathrm{H}$ NMR $\left(400 \mathrm{MHz}, \mathrm{CDCl}_{3}\right)$

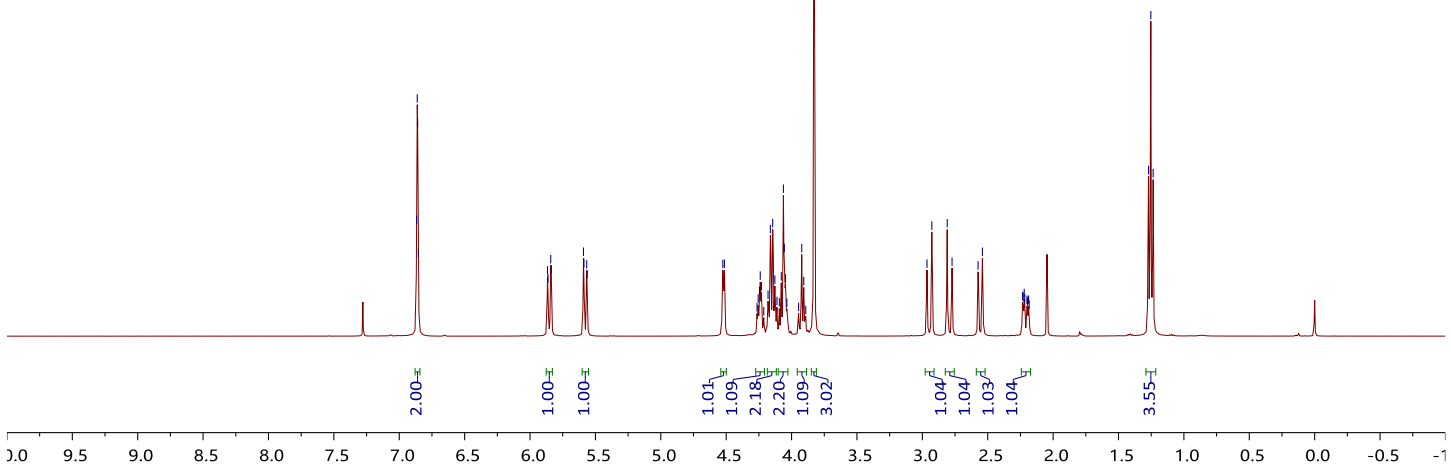

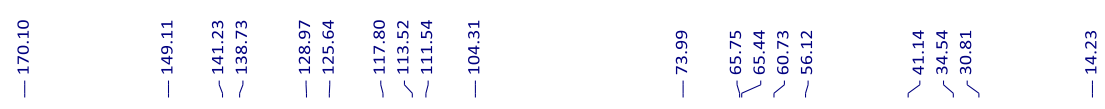

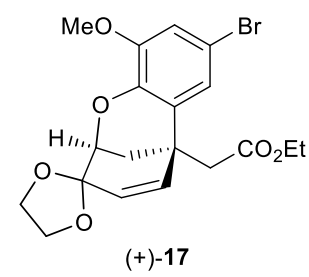

${ }^{13} \mathrm{C}$ NMR $\left(101 \mathrm{MHz}, \mathrm{CDCl}_{3}\right)$

oo 190
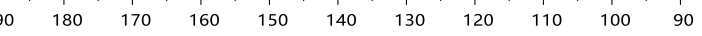

80

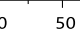




\section{Compound (+)-18}

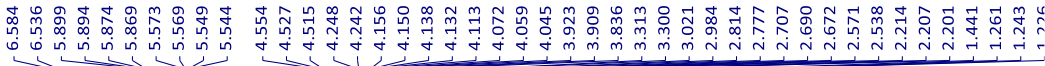

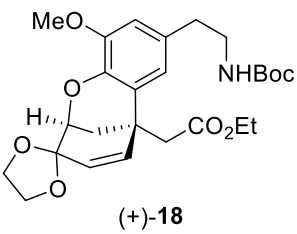

${ }^{1} \mathrm{H}$ NMR $\left(400 \mathrm{MHz}, \mathrm{CDCl}_{3}\right)$

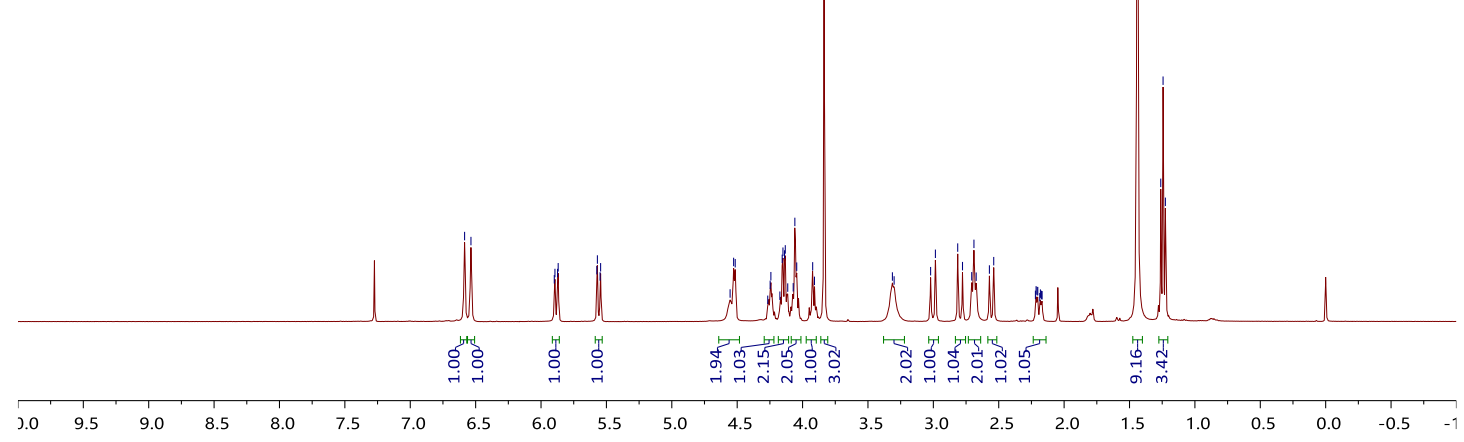

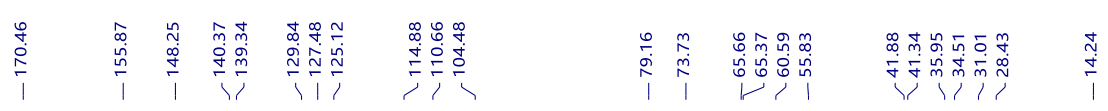

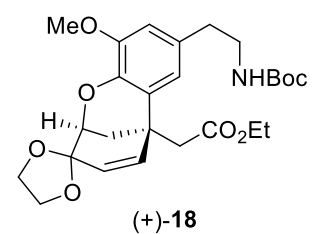

${ }^{13} \mathrm{C}$ NMR $\left(101 \mathrm{MHz}, \mathrm{CDCl}_{3}\right)$

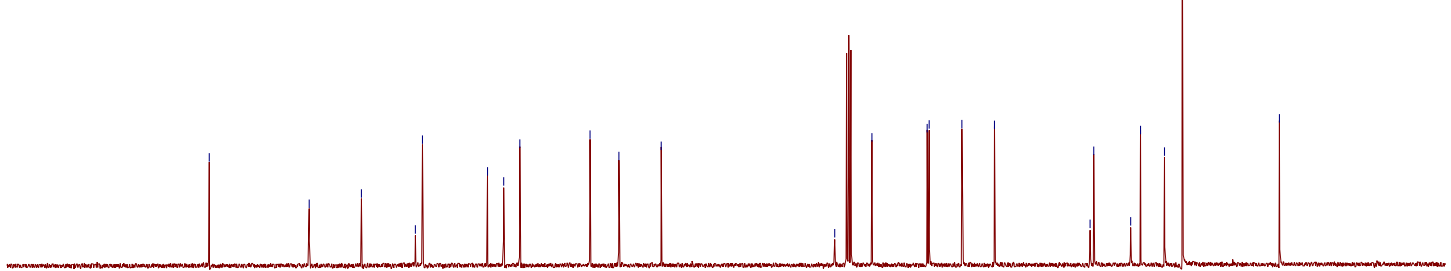

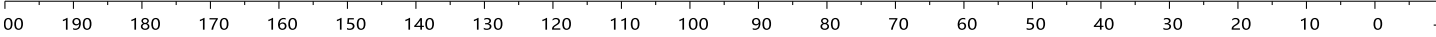




\section{Compound (+)-19}
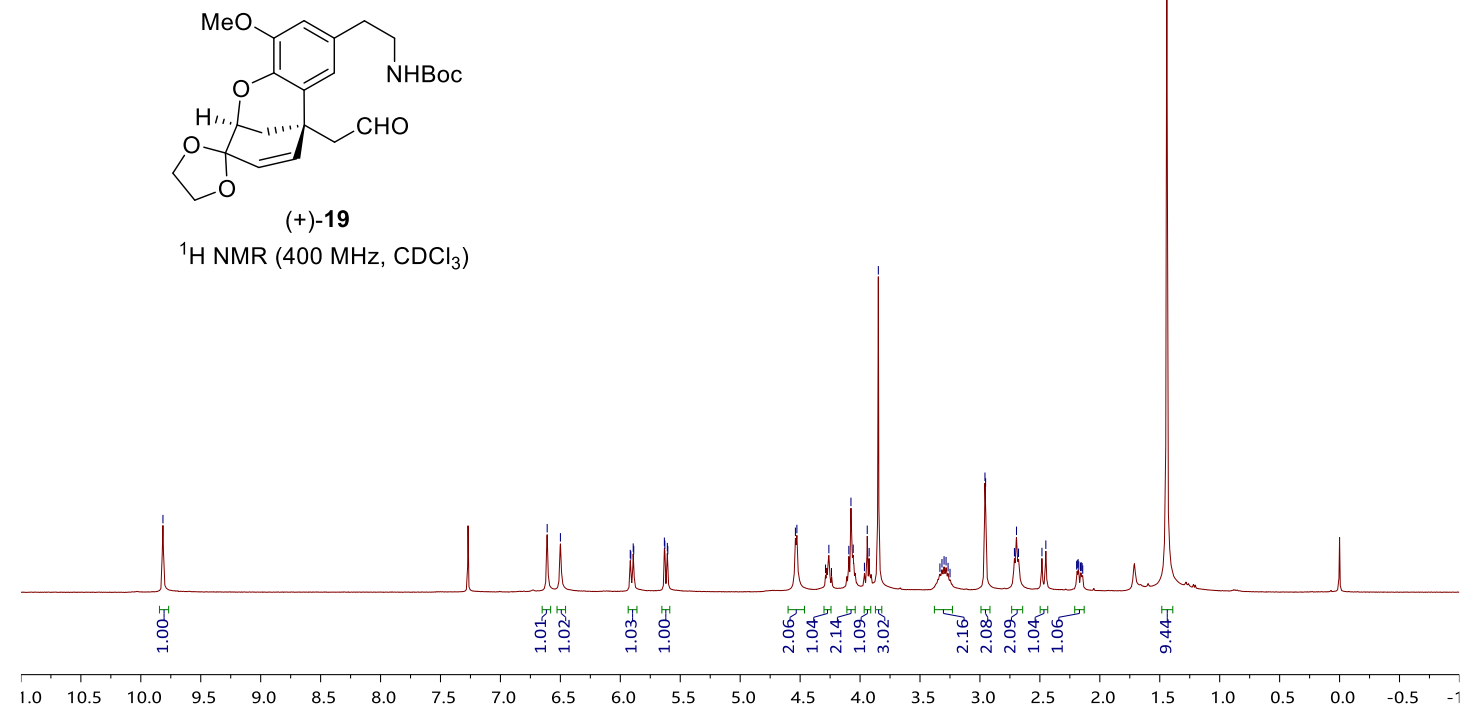

\begin{tabular}{|c|c|c|c|c|}
\hline 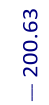 & 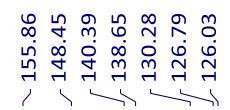 & 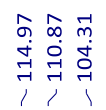 & 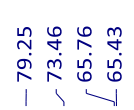 & 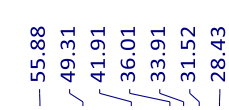 \\
\hline
\end{tabular}

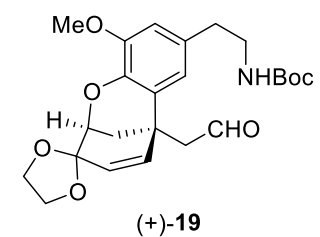

${ }^{13} \mathrm{C}$ NMR $\left(101 \mathrm{MHz}, \mathrm{CDCl}_{3}\right)$

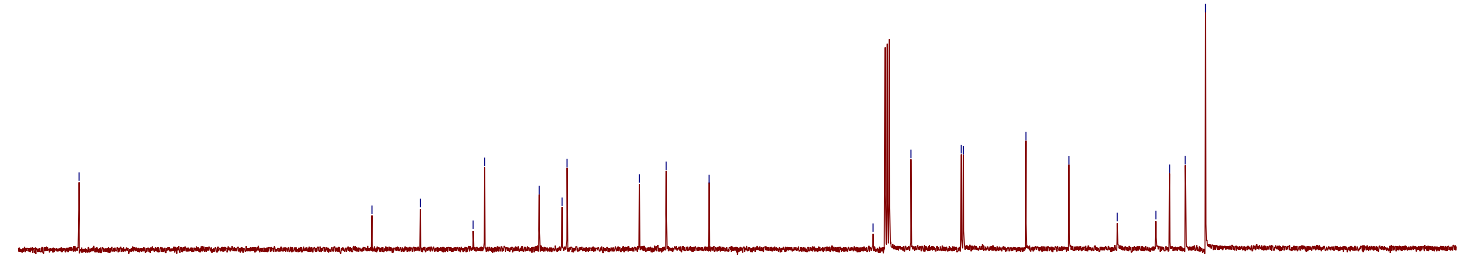

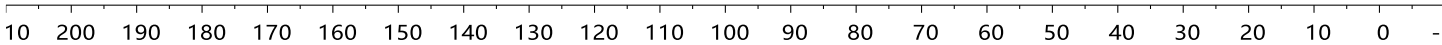




\section{Compound (+)-20}

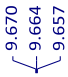

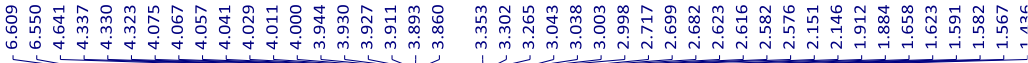

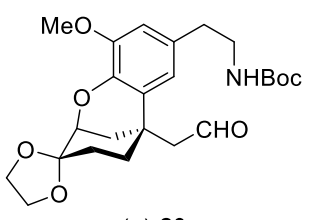

$(+)-20$

${ }^{1} \mathrm{H}$ NMR $\left(400 \mathrm{MHz}, \mathrm{CDCl}_{3}\right)$

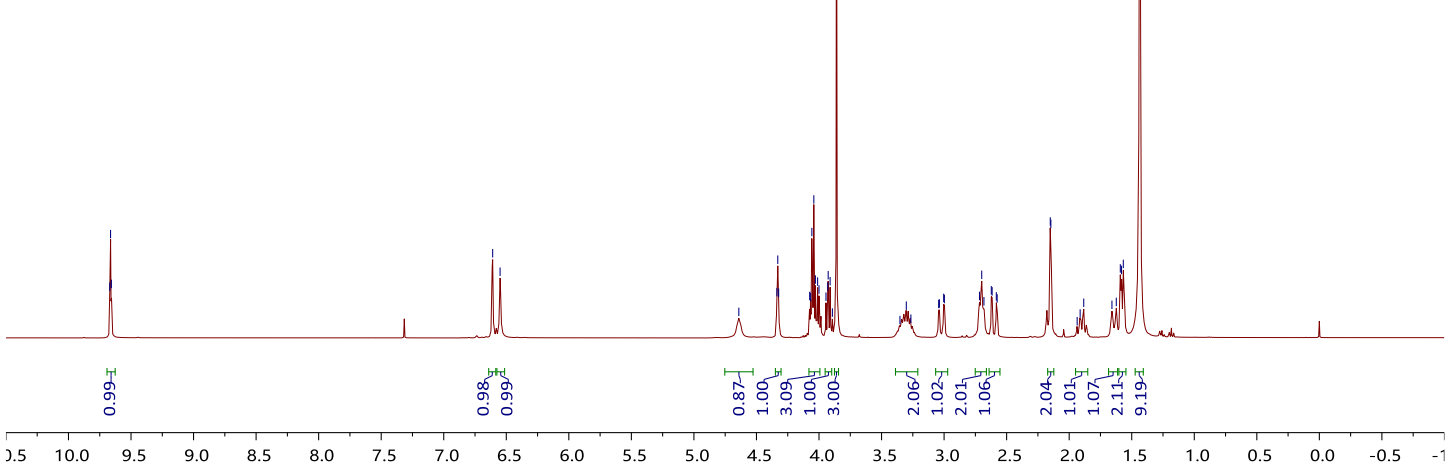

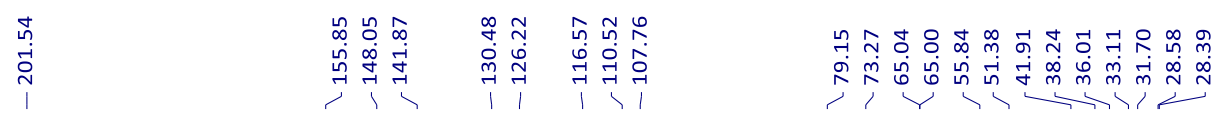

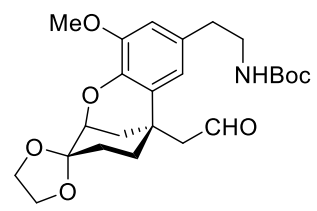

$(+)-20$

${ }^{13} \mathrm{C}$ NMR $\left(101 \mathrm{MHz}, \mathrm{CDCl}_{3}\right)$

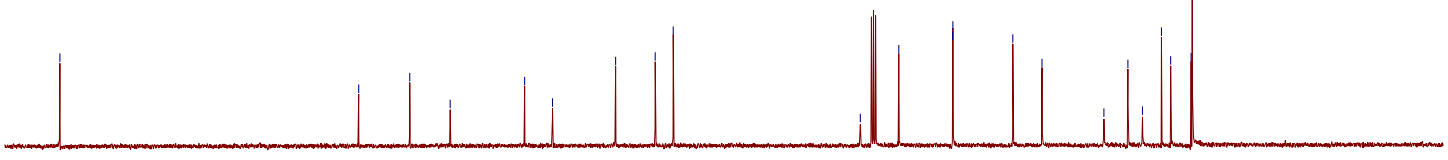

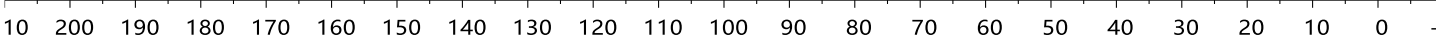


Compound (+)-21 (a mixture of $Z$ and $E$ isomers in a ratio of 44:56)

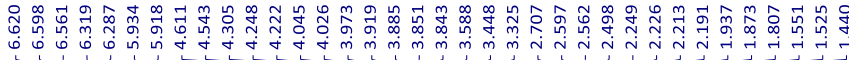

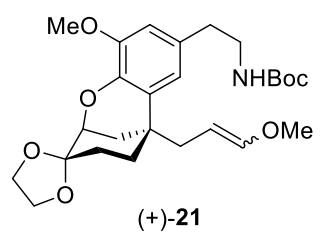

${ }^{1} \mathrm{H}$ NMR $\left(400 \mathrm{MHz}, \mathrm{CDCl}_{3}\right)$

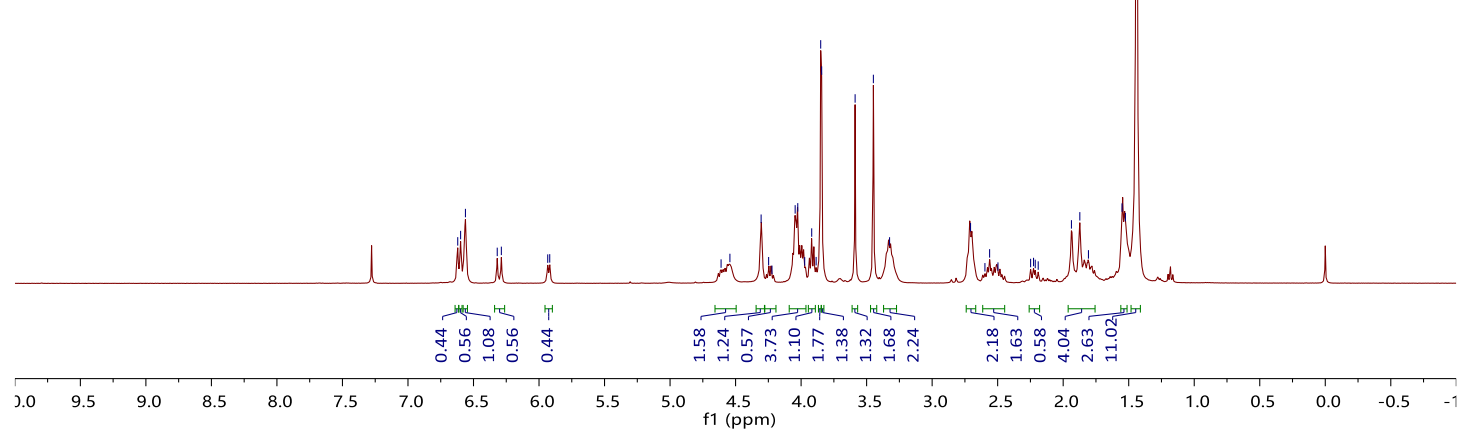

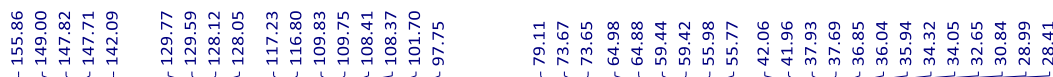

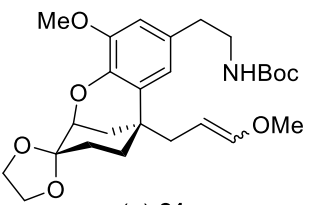

$(+)-21$

${ }^{13} \mathrm{C} \mathrm{NMR}\left(101 \mathrm{MHz}, \mathrm{CDCl}_{3}\right)$

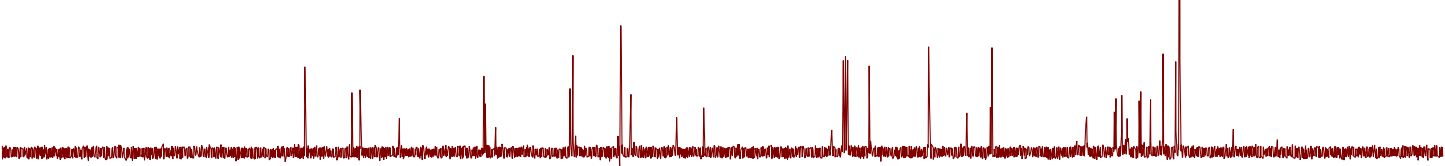

oo

$\begin{array}{llllllllll}180 & 170 & 160 & 150 & 140 & 130 & 120 & 110 & \begin{array}{c}100 \\ \mathrm{f} 1(\mathrm{ppm})\end{array}\end{array}$ 
Compound (+)-10 (with a minor diastereoisomer)

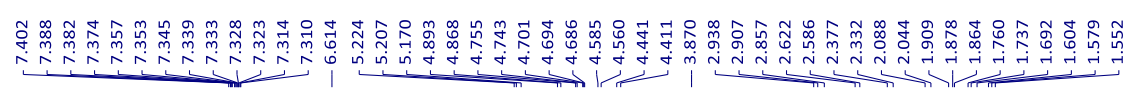

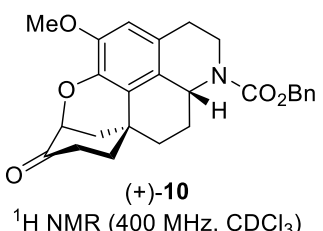

${ }^{1} \mathrm{H}$ NMR $\left(400 \mathrm{MHz}, \mathrm{CDCl}_{3}\right)$
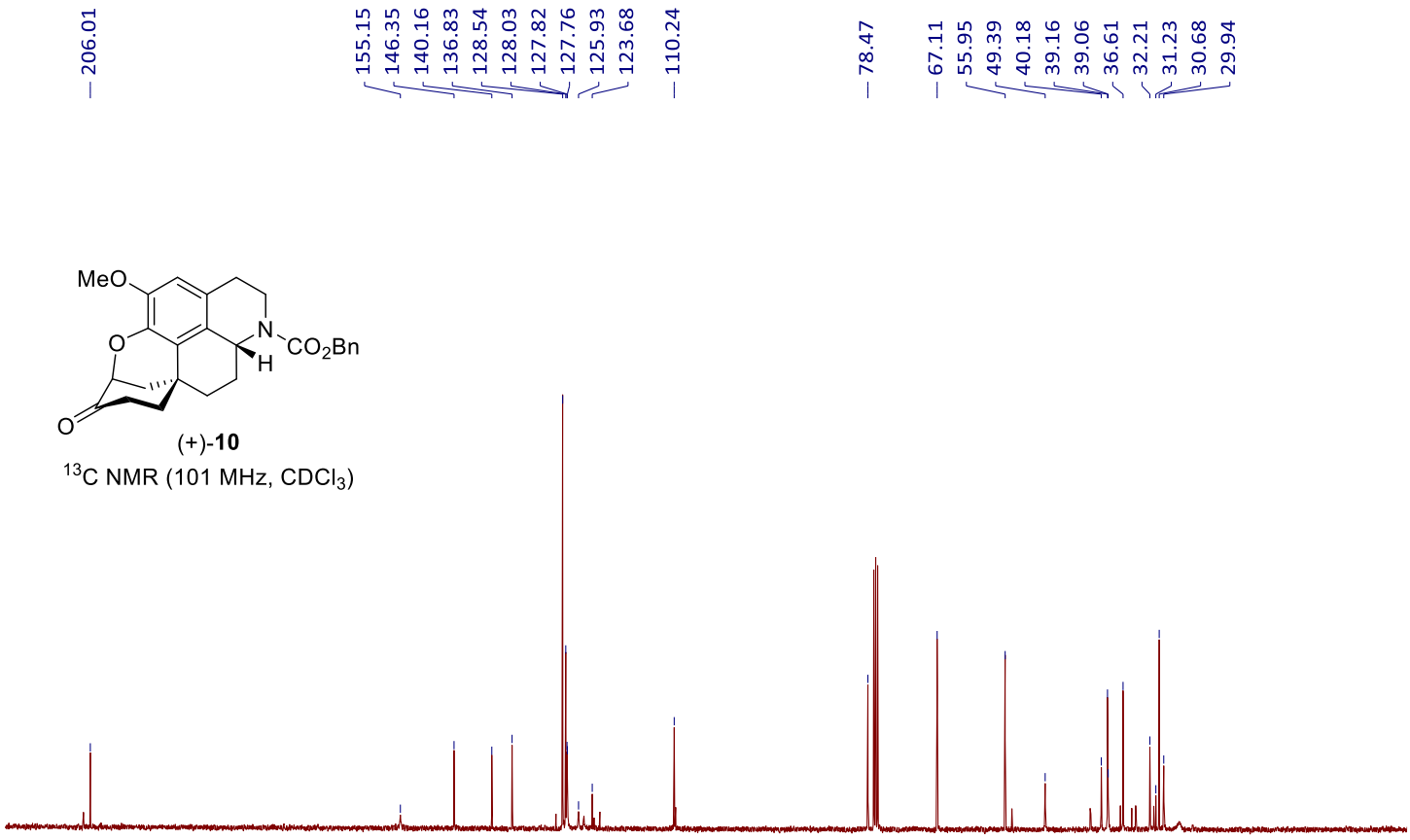

$\begin{array}{lllllllllllllllllllllll}210 & 200 & 190 & 180 & 170 & 160 & 150 & 140 & 130 & 120 & 110 & 100 & 90 & 80 & 70 & 60 & 50 & 40 & 30 & 20 & 10 & 0 & -\end{array}$ 
Compound (+)-24 (with a minor diastereoisomer)
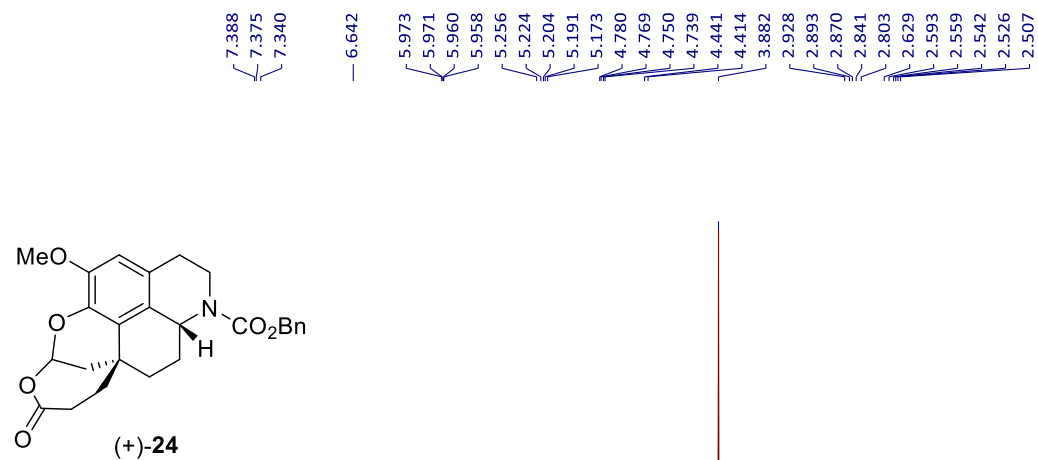

${ }^{1} \mathrm{H}$ NMR $\left(400 \mathrm{MHz}, \mathrm{CDCl}_{3}\right)$
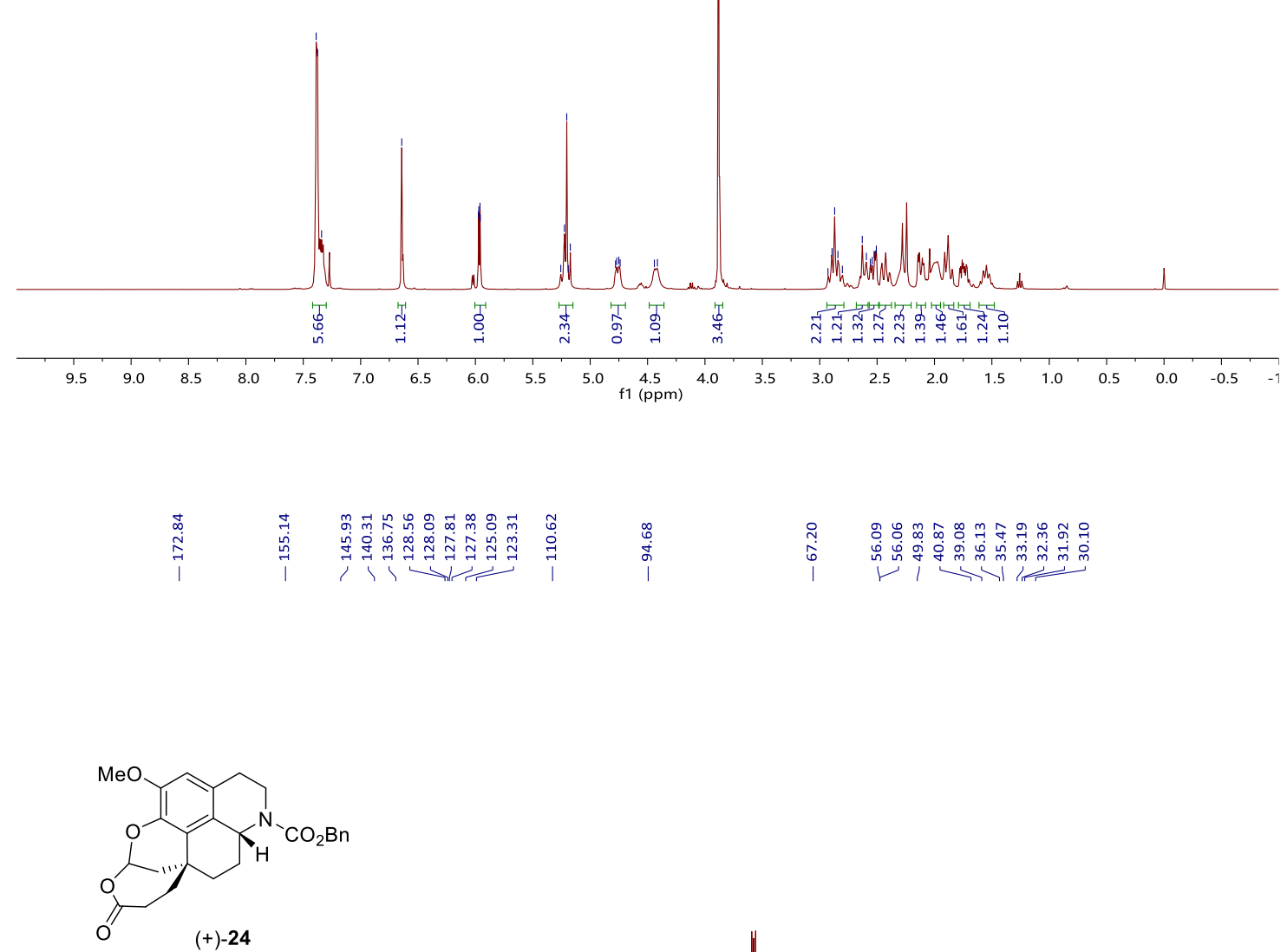

${ }^{13} \mathrm{C}$ NMR $\left(101 \mathrm{MHz}, \mathrm{CDCl}_{3}\right)$

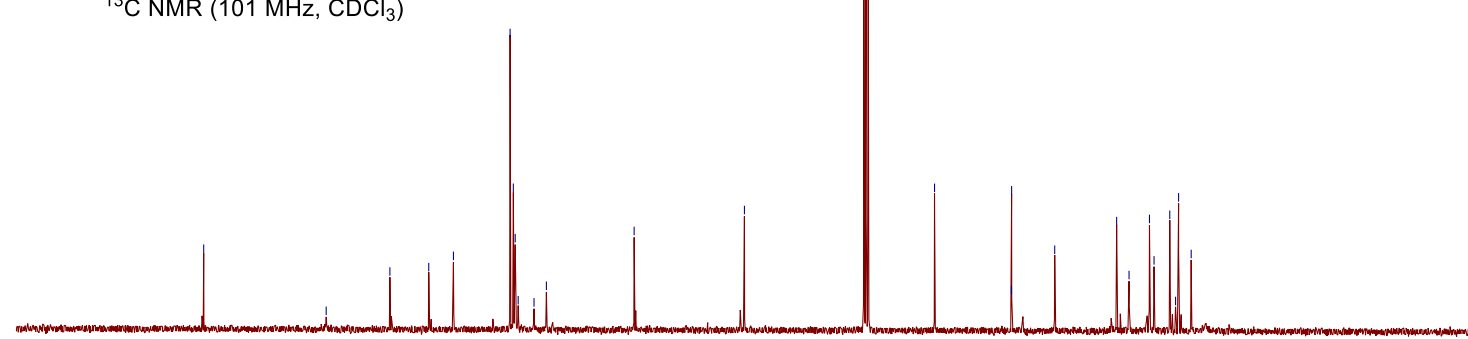

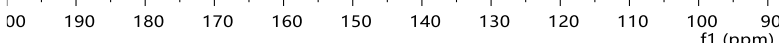


Compound (-)-25 (not stable, with minor diastereoisomers)
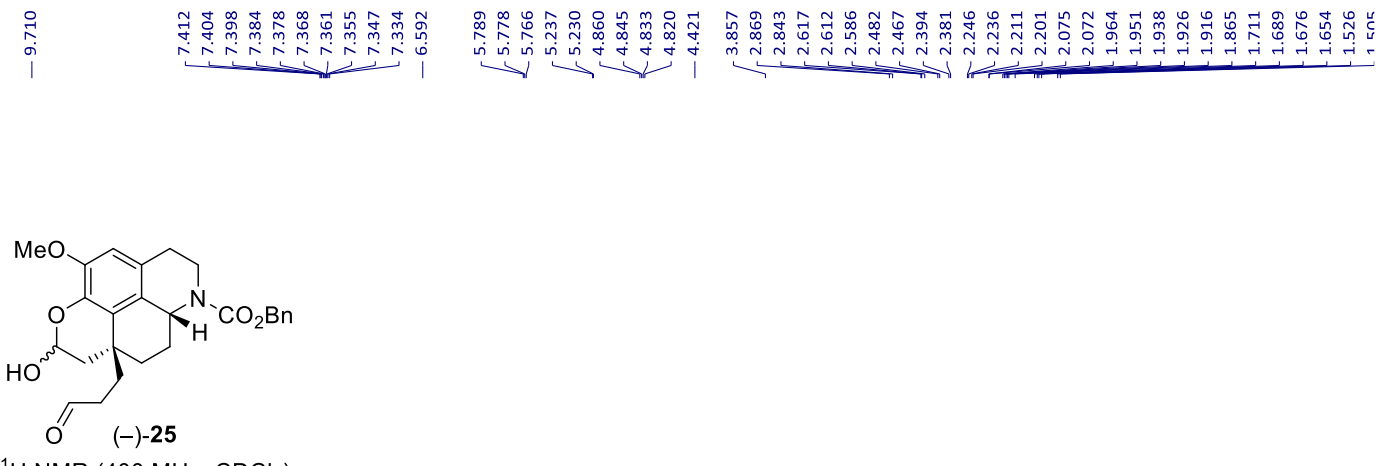

${ }^{1} \mathrm{H}$ NMR $\left(400 \mathrm{MHz}, \mathrm{CDCl}_{3}\right)$

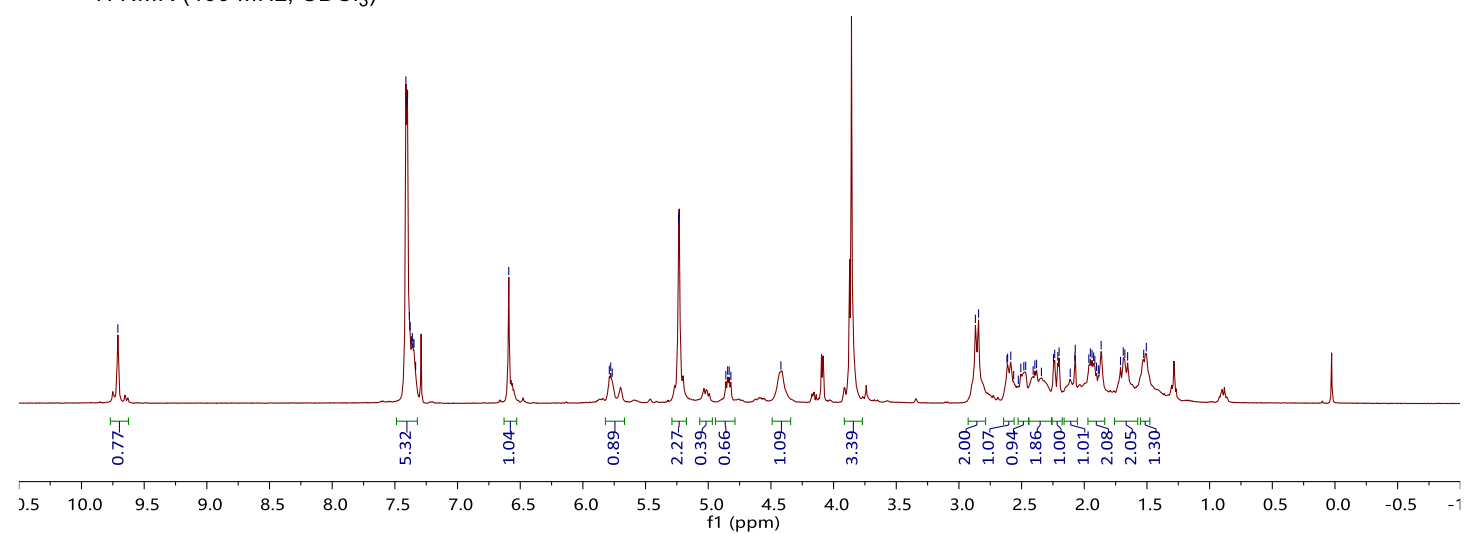

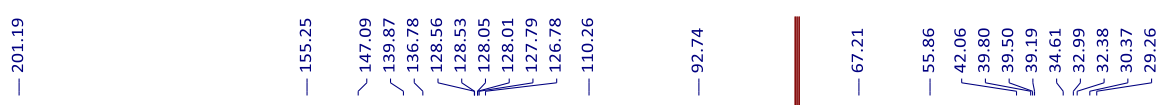

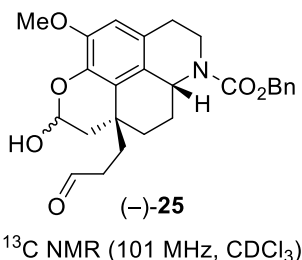

${ }^{13} \mathrm{C}$ NMR $\left(101 \mathrm{MHz}, \mathrm{CDCl}_{3}\right)$

$\begin{array}{llllllllllll}20 & 210 & 200 & 190 & 180 & 170 & 160 & 150 & 140 & 130 & 120 & \begin{array}{l}110 \\ \mathrm{f} 1(\mathrm{ppm})\end{array}\end{array}$ 
Compound (-)-26 (with a minor diastereoisomer)

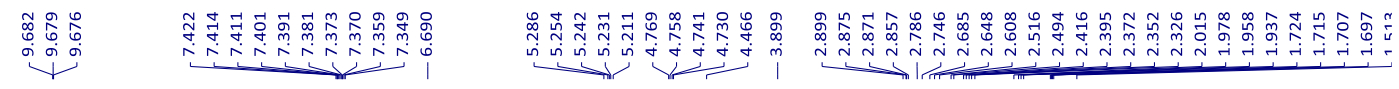

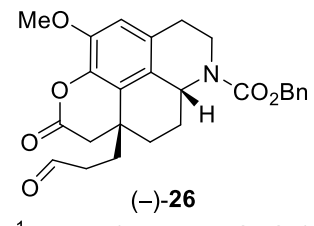

${ }^{1} \mathrm{H}$ NMR $\left(400 \mathrm{MHz}, \mathrm{CDCl}_{3}\right)$

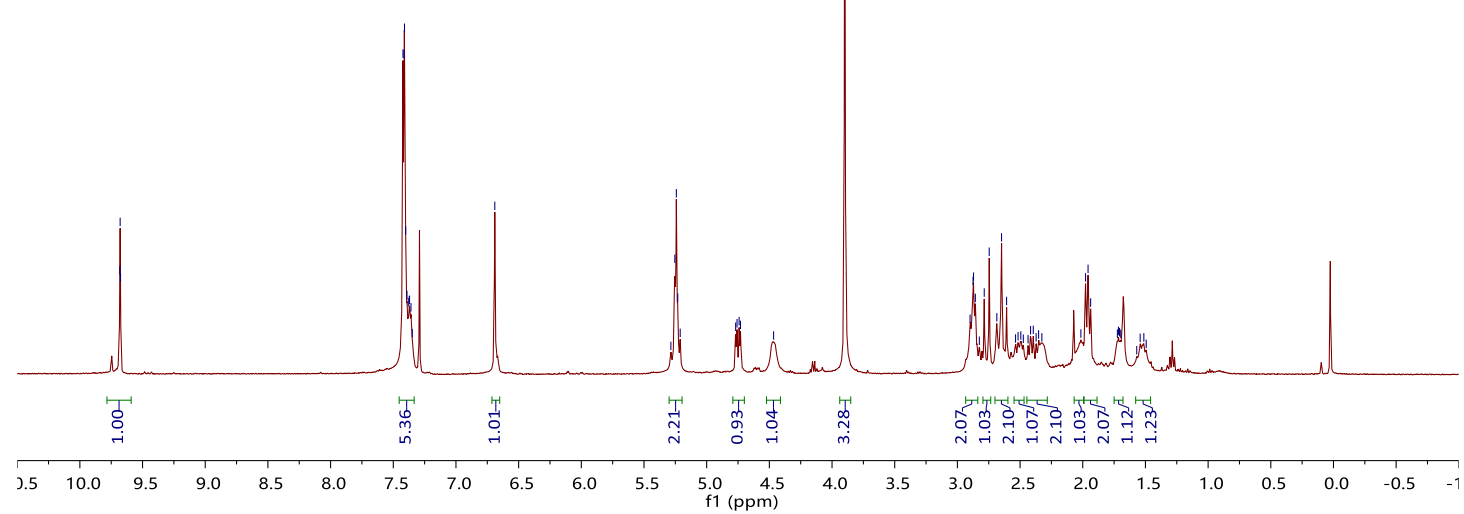

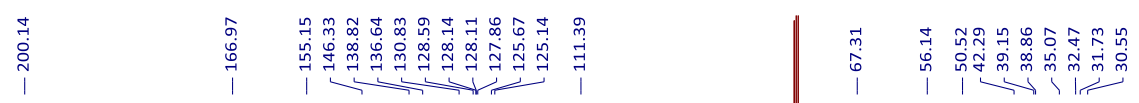

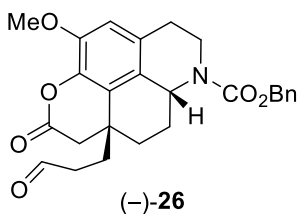

${ }^{13} \mathrm{C} \mathrm{NMR}\left(101 \mathrm{MHz}, \mathrm{CDCl}_{3}\right)$

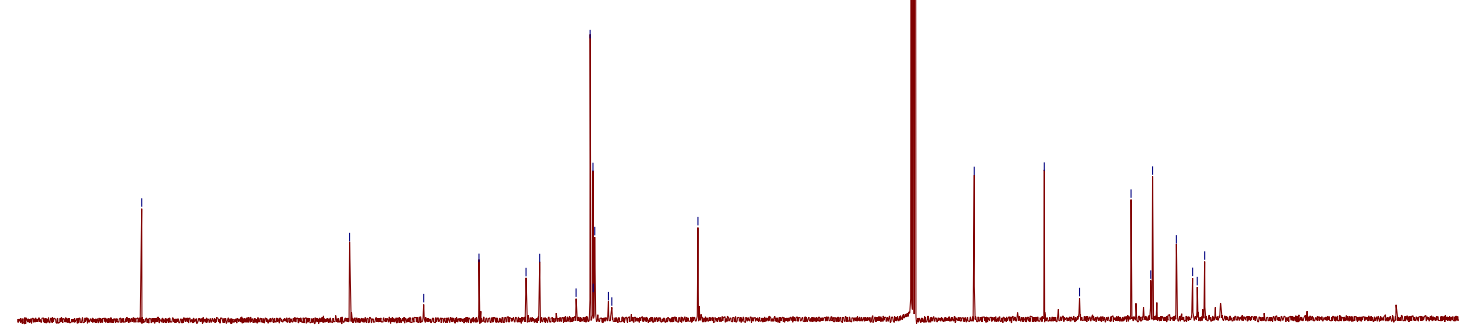

$\begin{array}{llllllllllll}20 & 210 & 200 & 190 & 180 & 170 & 160 & 150 & 140 & 130 & 120 & \begin{array}{l}110 \\ \mathrm{f} 1(\mathrm{ppm})\end{array}\end{array}$ 


\section{Compound (-)-9}

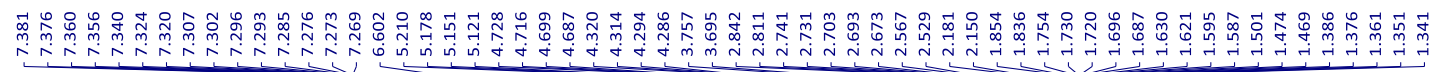
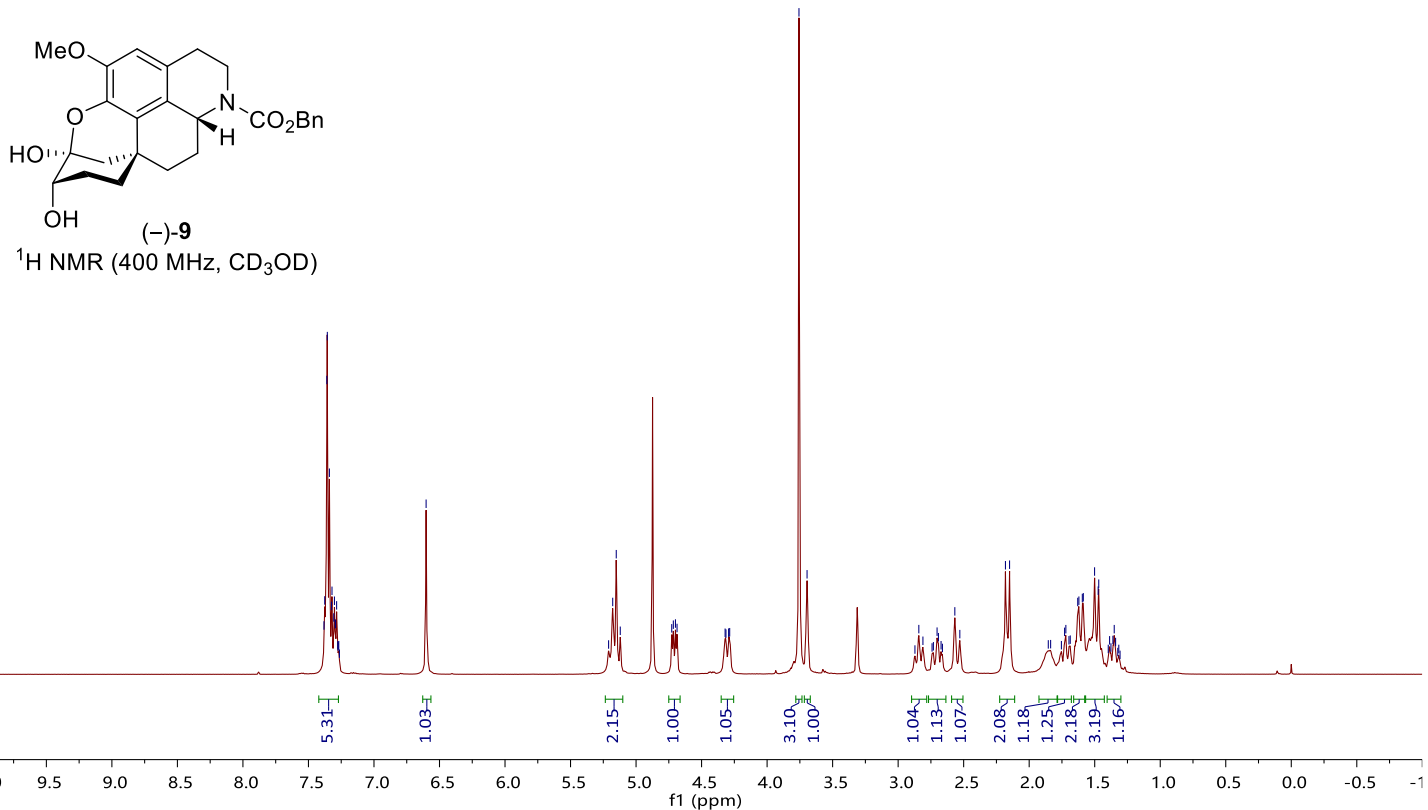

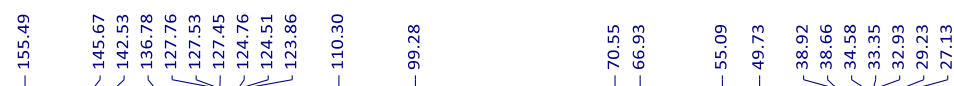

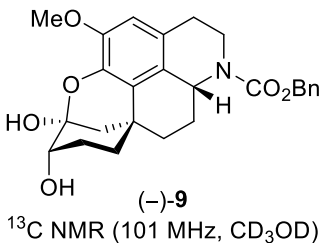

${ }^{13} \mathrm{C}$ NMR $\left(101 \mathrm{MHz}, \mathrm{CD}_{3} \mathrm{OD}\right)$

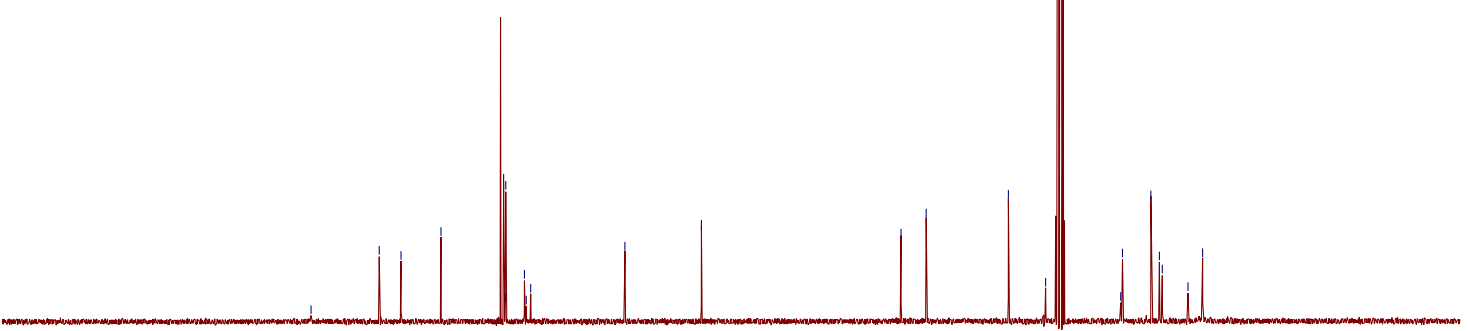

$\begin{array}{llllllllll}190 & 180 & 170 & 160 & 150 & 140 & 130 & 120 & 110 & \begin{array}{c}100 \\ f 1(\mathrm{ppm})\end{array}\end{array}$ 
Compound (-)-27 (with a minor diastereoisomer)
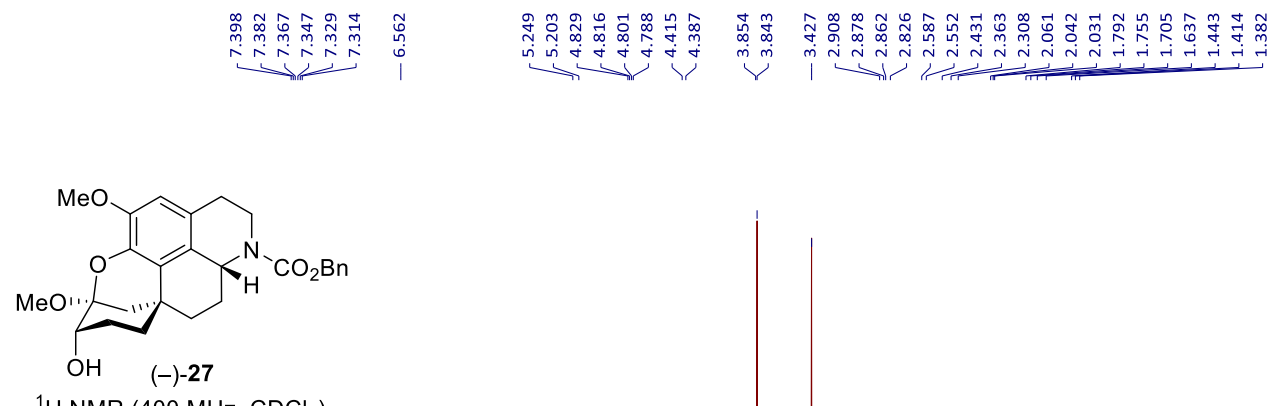

${ }^{1} \mathrm{H}$ NMR $\left(400 \mathrm{MHz}, \mathrm{CDCl}_{3}\right)$
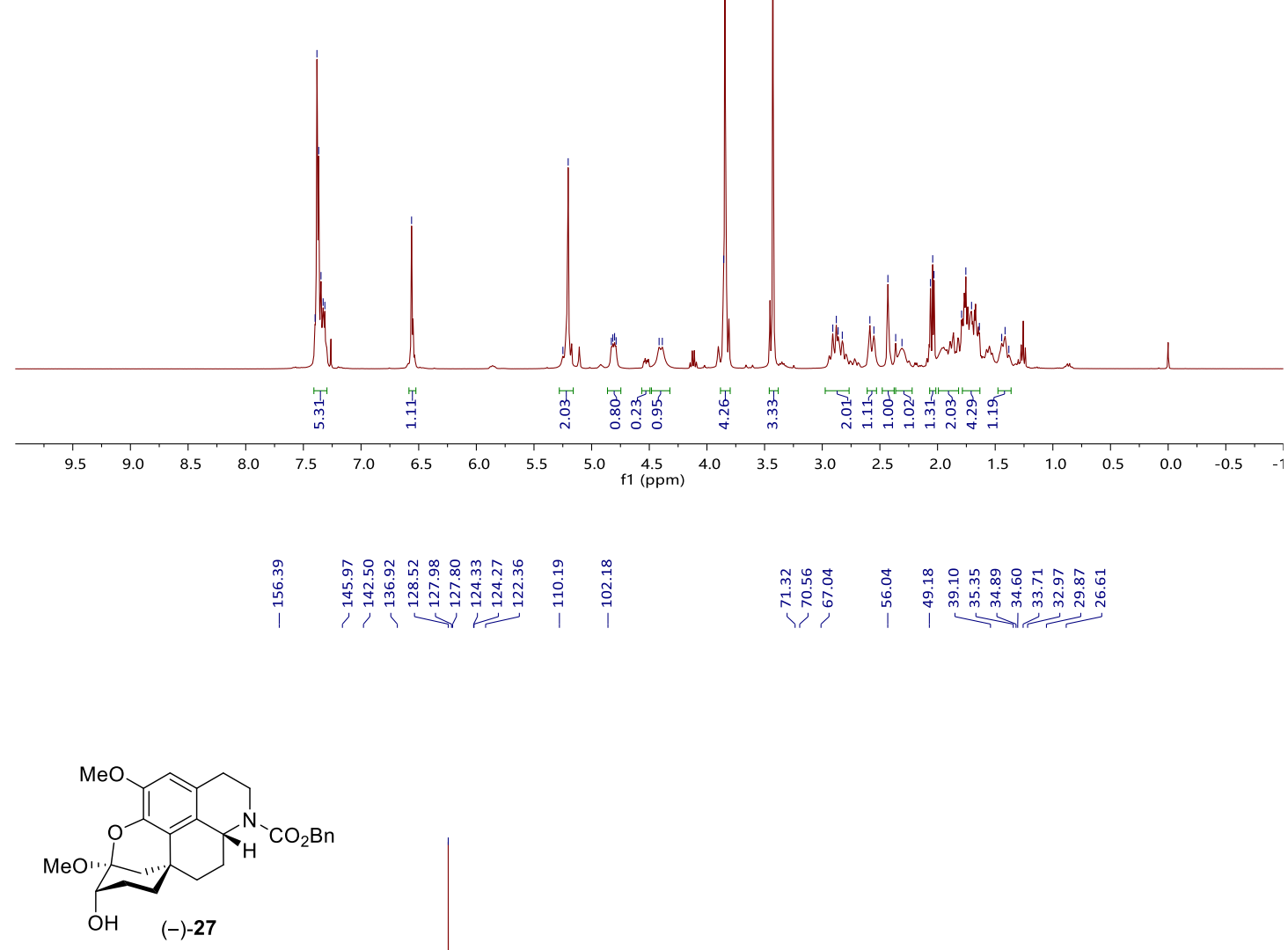

${ }^{13} \mathrm{C}$ NMR $\left(101 \mathrm{MHz}, \mathrm{CDCl}_{3}\right)$

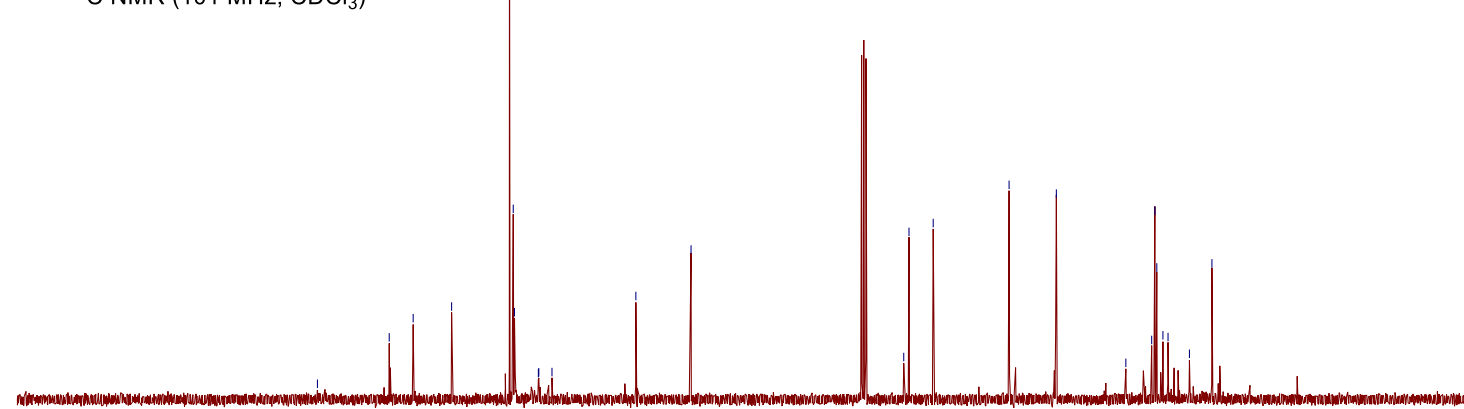
$\begin{array}{lllllllllll}00 & 190 & 180 & 170 & 160 & 150 & 140 & 130 & 120 & 110 & 100 \begin{array}{r}90 \\ \mathrm{f} 1(\mathrm{ppm})\end{array}\end{array}$ 
(+)-Regeline (1)

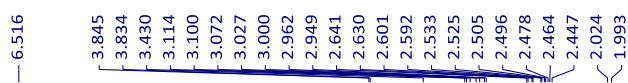

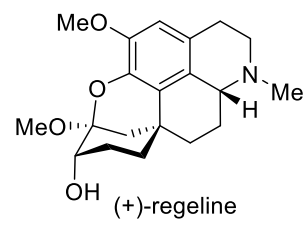

${ }^{1} \mathrm{H}$ NMR $\left(400 \mathrm{MHz}, \mathrm{CDCl}_{3}\right)$
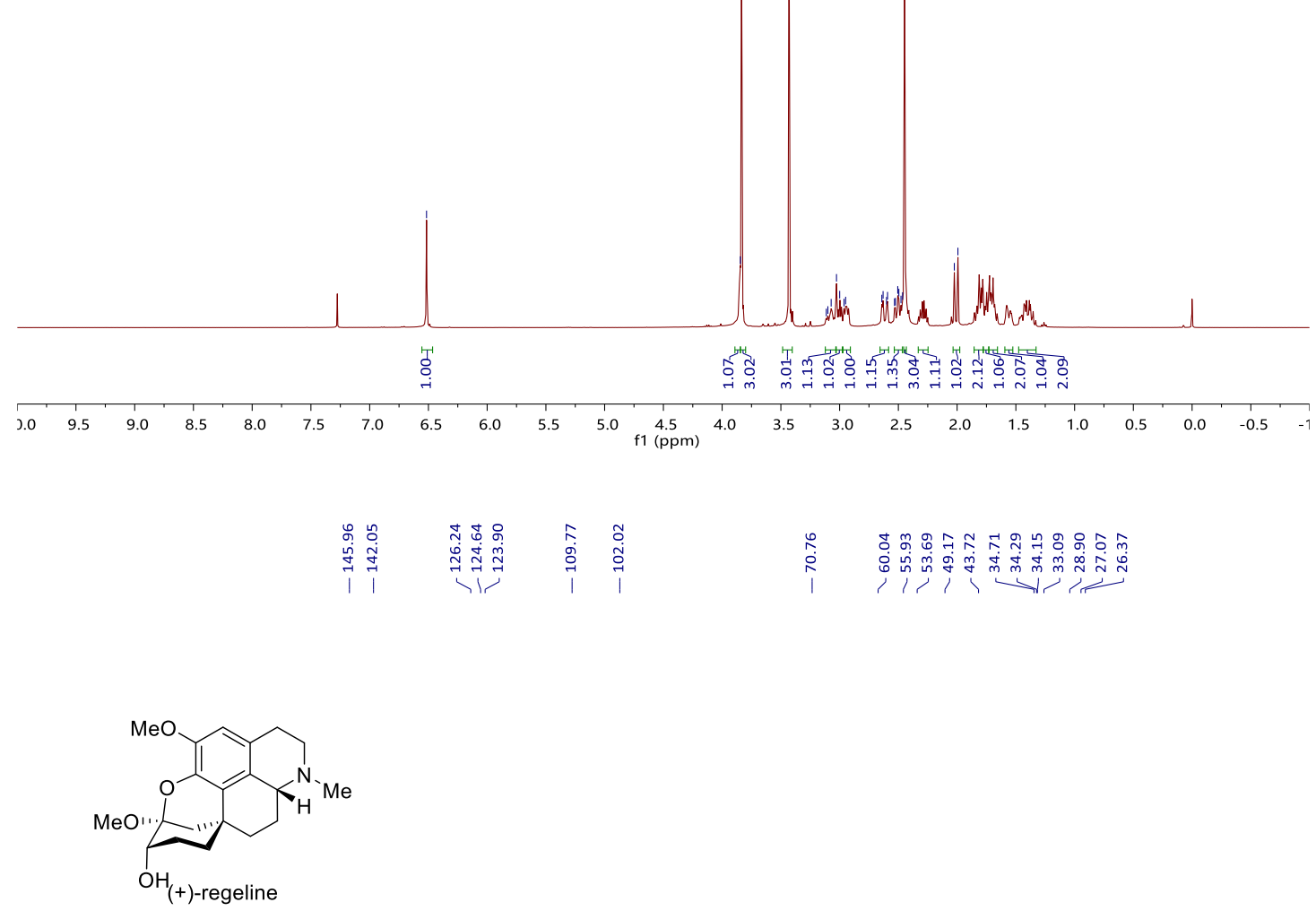

${ }^{13} \mathrm{C}$ NMR $\left(101 \mathrm{MHz}, \mathrm{CDCl}_{3}\right)$

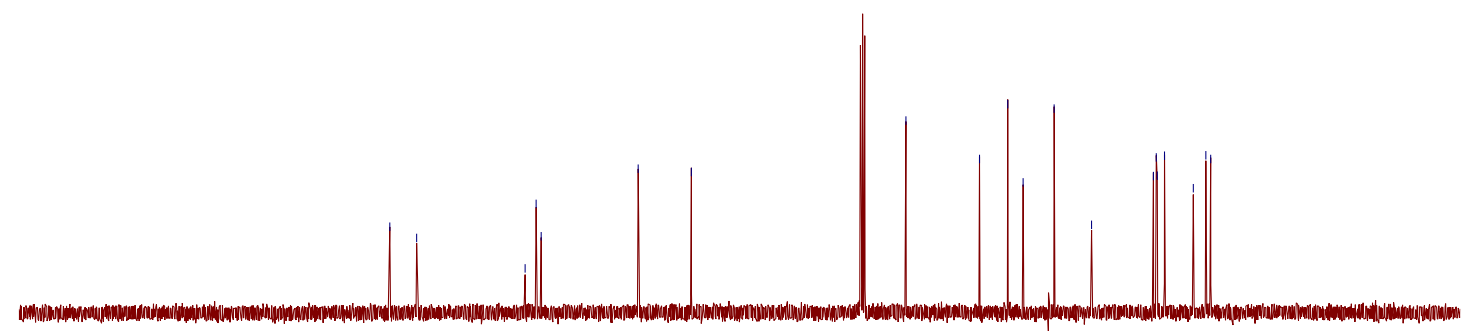

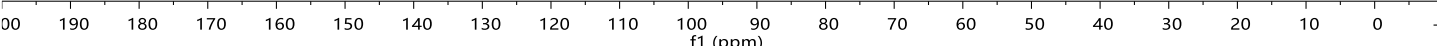


(+)-Regalamine (2)

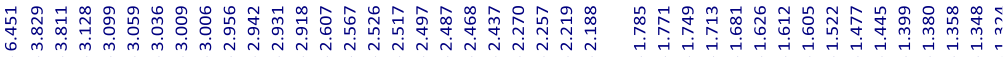

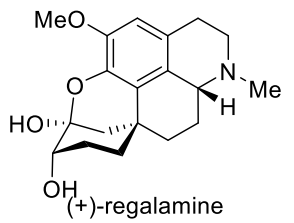

${ }^{1} \mathrm{H}$ NMR (400 MHz, $\mathrm{CDCl}_{3}$ )

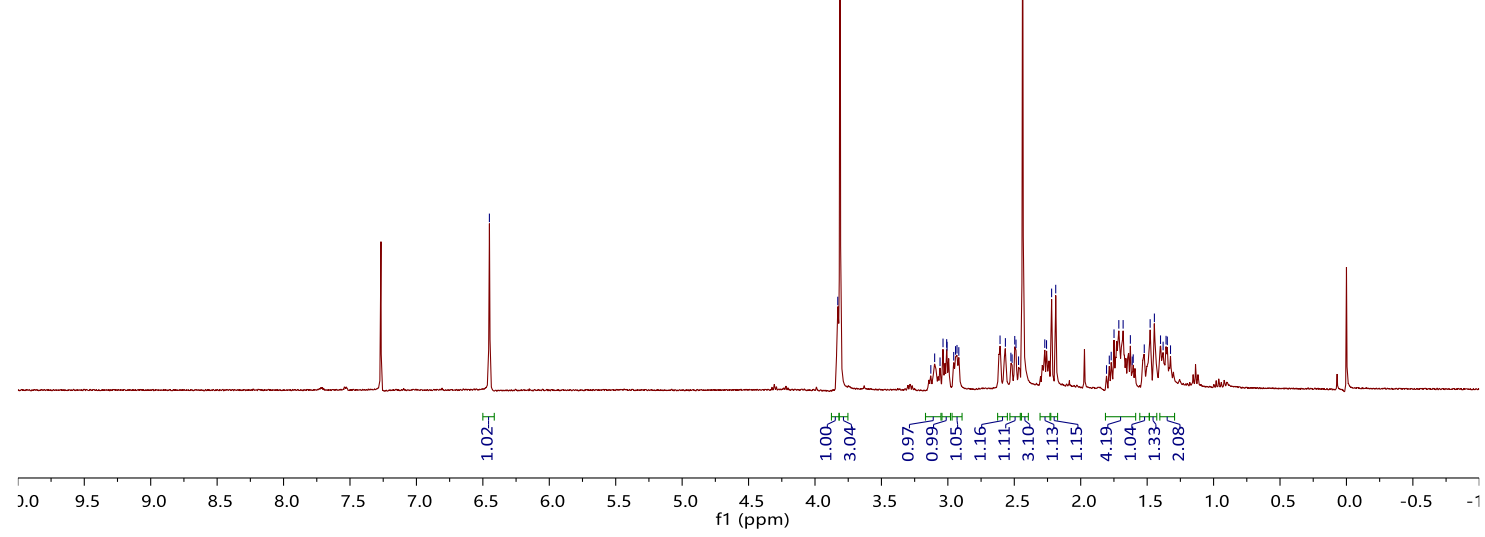

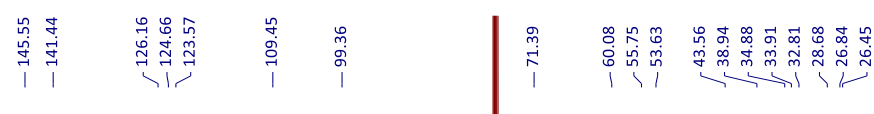

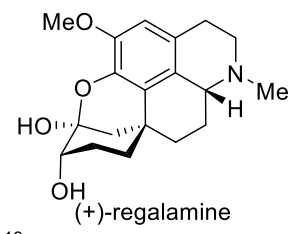

${ }^{13} \mathrm{C}$ NMR $\left(101 \mathrm{MHz}, \mathrm{CDCl}_{3}\right)$

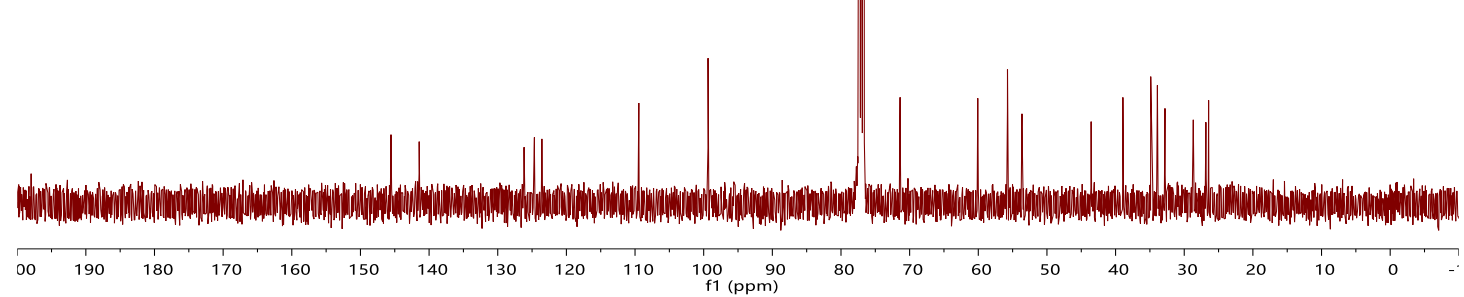


(+)-Kesselridine (3)

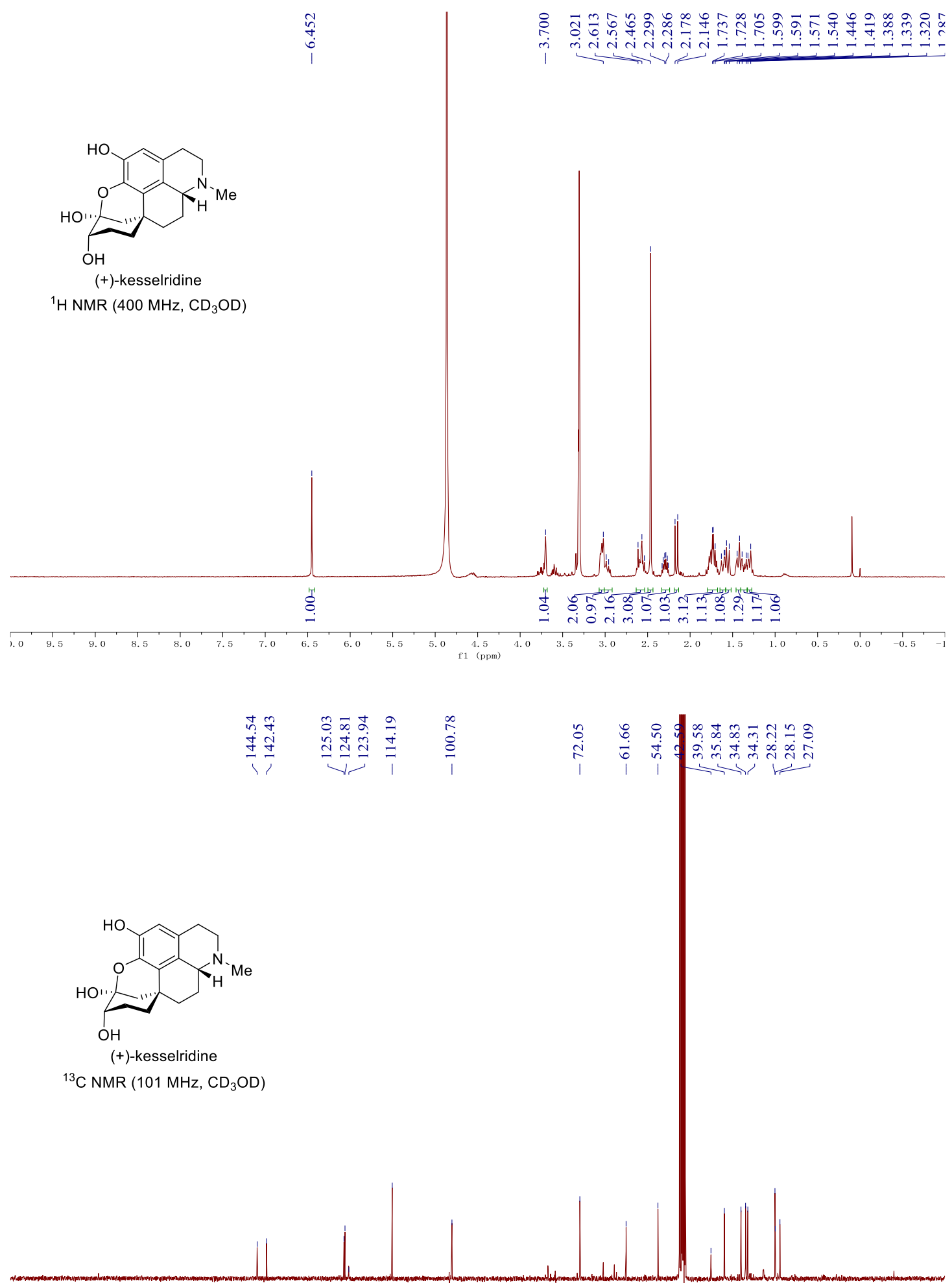

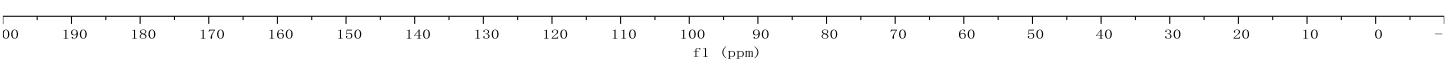


(+)-Kesselringine (4)
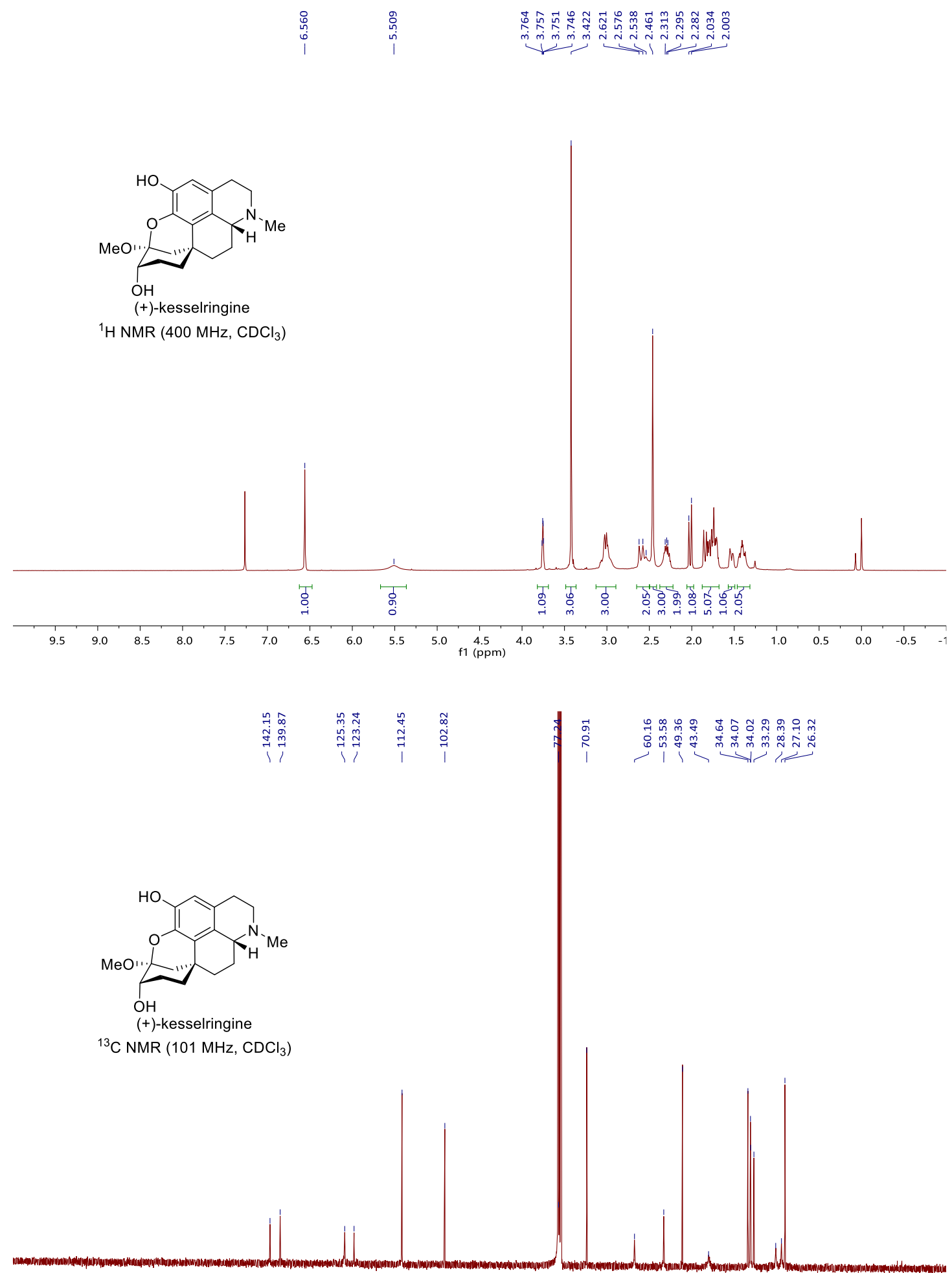

$\begin{array}{llllllllllllllllllll}190 & 180 & 170 & 160 & 150 & 140 & 130 & 120 & 110 & \begin{array}{c}100 \\ \mathrm{f} 1(\mathrm{ppm})\end{array} & 80 & 70 & 60 & 50 & 40 & 30 & 20 & 10 & 0 & \end{array}$ 
(+)-Jolantidine (5)
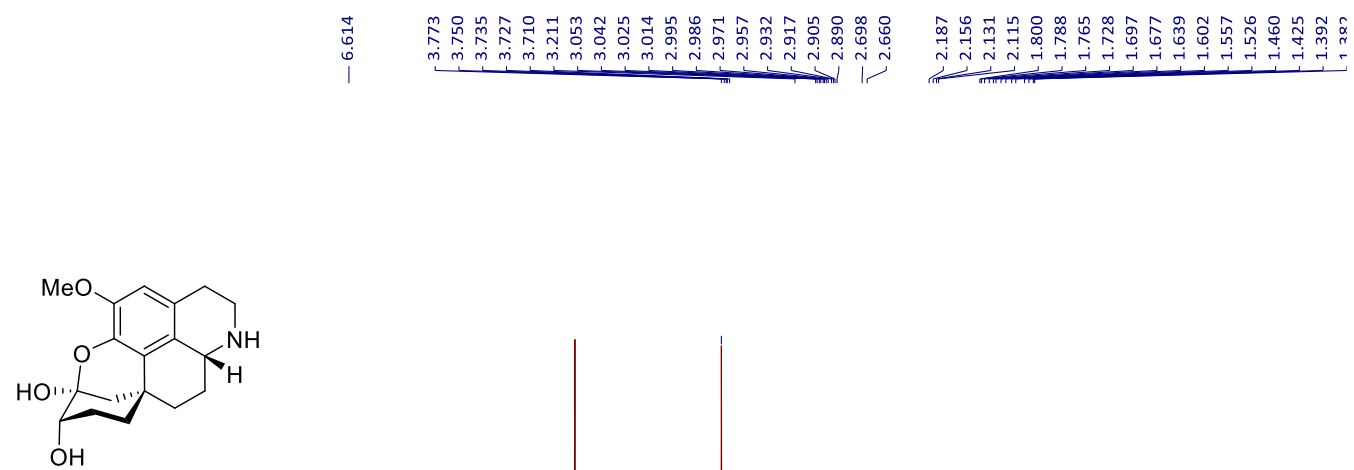

$(+)$-jolantidine

${ }^{1} \mathrm{H}$ NMR (400 MHz, $\mathrm{CD}_{3} \mathrm{OD}$ )

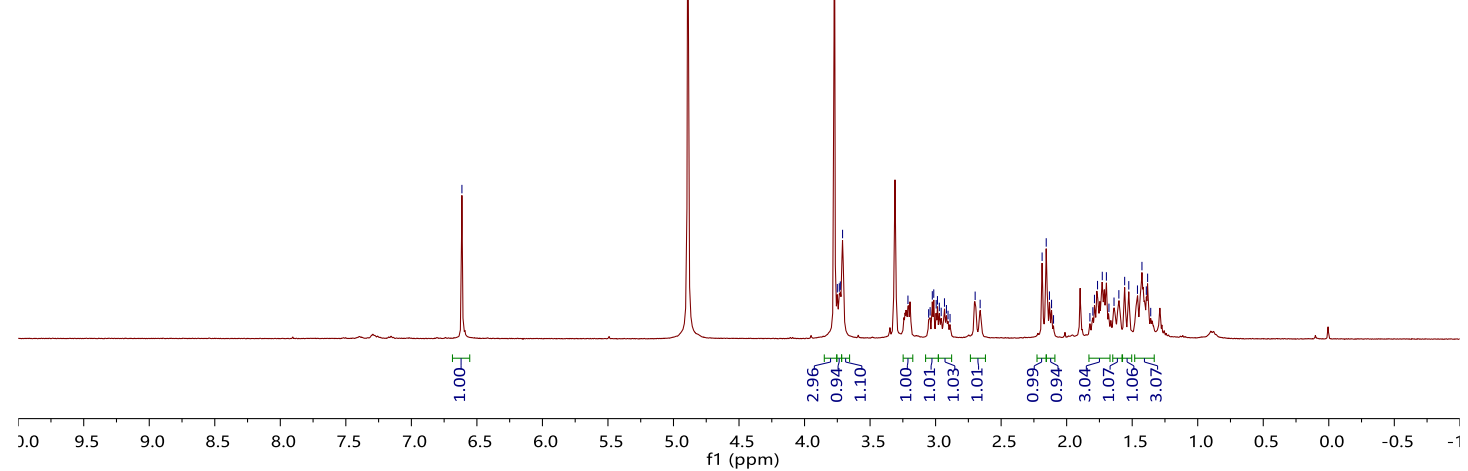

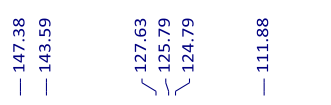
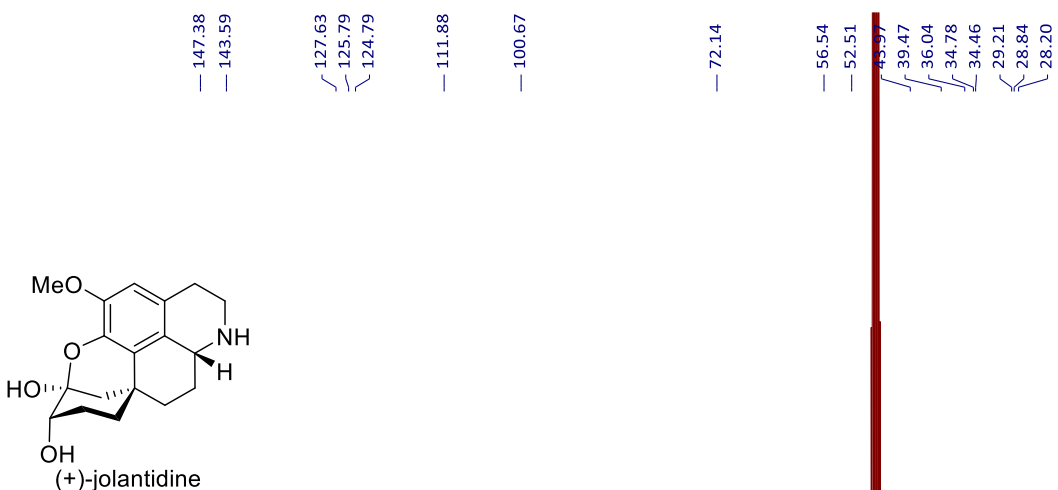

${ }^{13} \mathrm{C}$ NMR (101 MHz, $\mathrm{CD}_{3} \mathrm{OD}$ ) 
(+)-Regelinine (6)

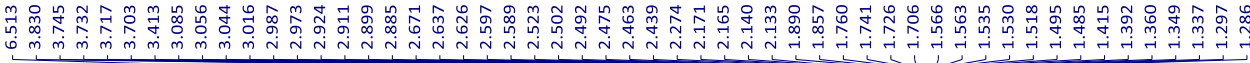

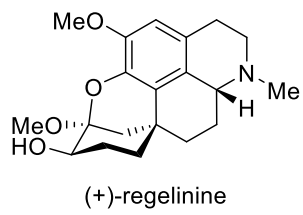

${ }^{1} \mathrm{H}$ NMR $\left(400 \mathrm{MHz}, \mathrm{CDCl}_{3}\right)$

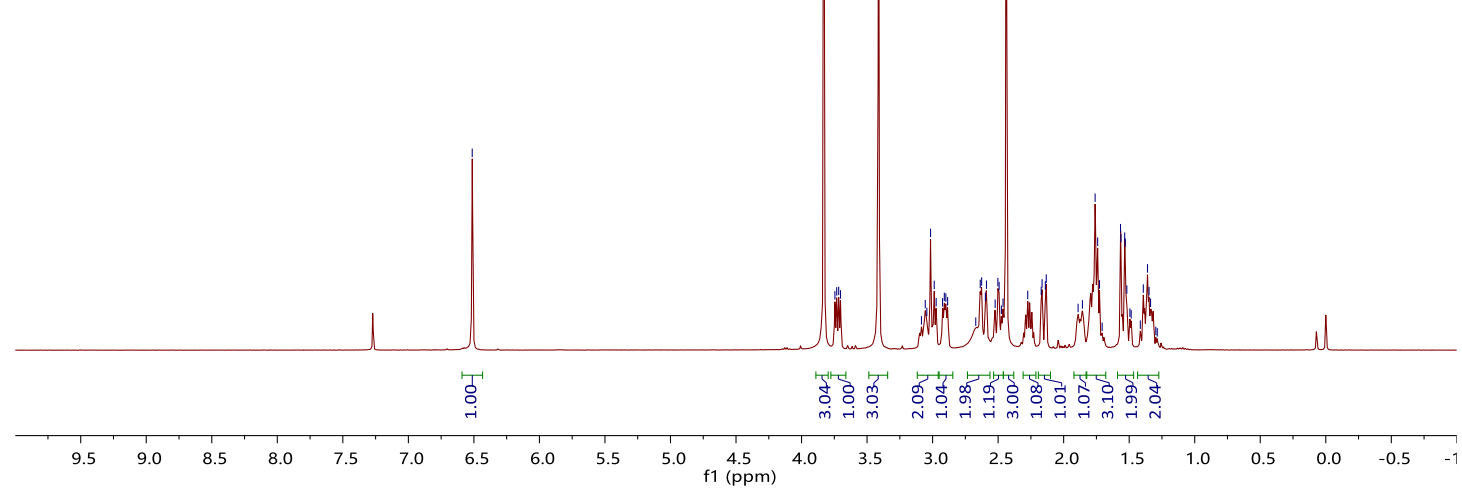

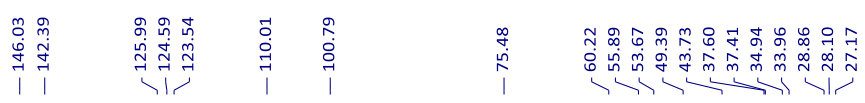

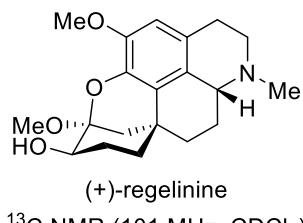

${ }^{13} \mathrm{C}$ NMR $\left(101 \mathrm{MHz}, \mathrm{CDCl}_{3}\right)$

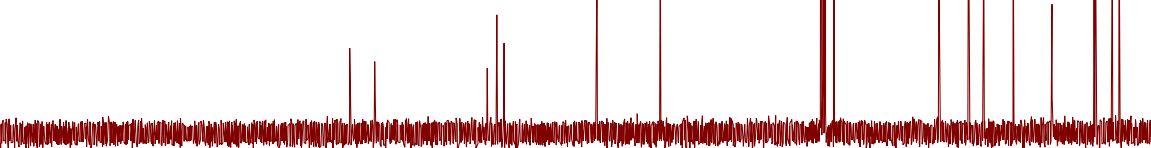

$\begin{array}{llllllllll}190 & 180 & 170 & 160 & 150 & 140 & 130 & 120 & 110 & \begin{array}{l}100 \\ \mathrm{f} 1(\mathrm{ppm})\end{array}\end{array}$ 
F) HPLC Charts for (-)-13
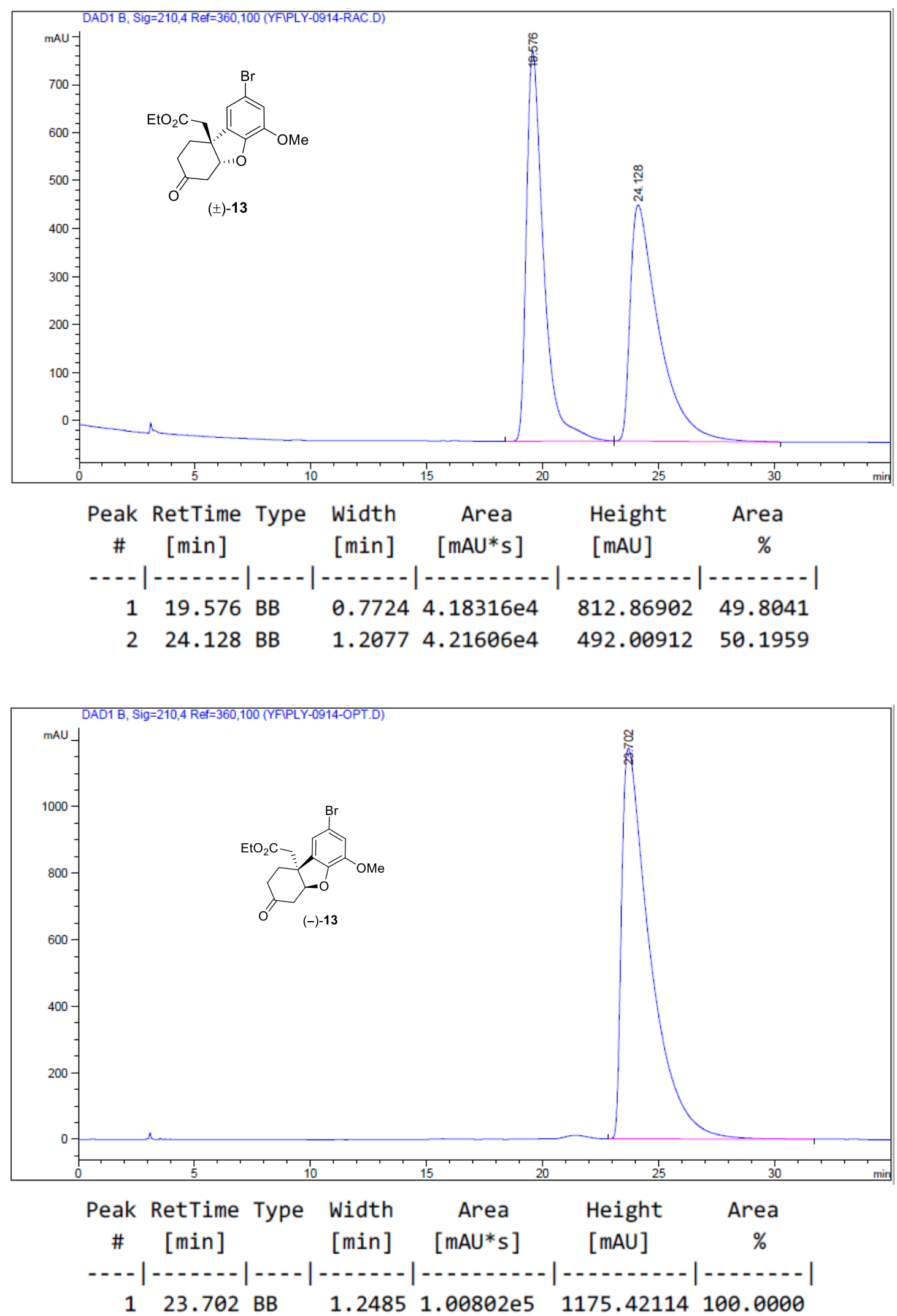